\title{
V. GRAPE MEASUREMENTS OF WET BULK DENSITY AND POROSITY, LEG 43
}

\author{
The Shipboard Scientific Party ${ }^{1}$
}

The following computer listing (Table 1) gives routine Gamma Ray Attenuation Porosity Evaluator (GRAPE) determinations of wet bulk density and porosity for core sections obtained during DSDP Leg 43. The calculations assumed a grain density of 2.65 $\mathrm{g} / \mathrm{cm}^{3}$. With four exceptions (Site 384, Core 22, Sections 1 and 2; Site 385, Core 20, Section 2; Site 386, Core 66, Section 1), diameter measurements were not made on core sections and most GRAPE values therefore are not corrected for diameter variations. Thus all GRAPE values should be carefully screened by study of the corresponding core photographs. Detailed descriptions of how to interpret the GRAPE data, the methods used to determine GRAPE density and porosity, and the limitations on the usefulness of the data are given by Boyce (1976).

${ }^{1}$ Brian E. Tucholke (Co-Chief Scientist), Lamont-Doherty Geological Observatory, Palisades, New York; Peter R. Vogt (Co-Chief Scientist), Naval Research Laboratory, Washington, D.C.; Kenneth R. Demars, University of Rhode Island, Kingston, Rhode Island (now at: University of Delaware, Newark, Delaware); Jon S. Galehouse, San Francisco State University, San Francisco, California; Robert L. Houghton, Woods Hole Oceanographic Institution, Woods Hole, Massachusetts (now at: University of Alberta, Edmonton, Alberta, Canada); Ansis Kaneps, Deep Sea Drilling Project, Scripps Institution of Oceanography, La Jolla, California; John W. Kendrick, Shell Development Company, Houston, Texas; I. N. McCave, University of East Anglia, Norwich, England; Charles L. McNulty, University of Texas, Arlington, Texas; Ivar O. Murdmaa, P. P. Shirshov Institute of Oceanology, Moscow, USSR; Hisatake Okada, Lamont-Doherty Geological Observatory, Palisades, New York (now at: Yamagata University, Yamagata, Japan); and Peter Rothe, Mineralogisch Petrografisches Institut der Universität Heidelberg, Heidelberg, Federal Republic of Germany (now at: Geographisches Institut der Universität Mannheim, Manheim, Federal Republic of Germany).
In the listing, the first line gives the site number, core number, section number, and sub-bottom depth to the top of the section, followed by a code: $0>$ [assumed grain density in $\mathrm{g}$ per $\mathrm{cm}^{3} /$ assumed or measured sample diameter in $\mathrm{cm}$ and a notation, $\mathrm{C} 0$, indicating the core sample was centered on the gamma-ray beam/assumed density in $\mathrm{g}$ per cc of any slurry surrounding the solid core/disturbance notation, 4 , which is an artifact of the program and of no significance here]. Where the code begins with $0>(0$ or another number being depth in section in $\mathrm{cm}$ ), the subsequent parameters are all nominal values; $0=$ precedes parameters that include measured diameters but are otherwise nominal values. The values of wet bulk density $\left(\mathrm{Rho}, \mathrm{g} / \mathrm{cm}^{3}\right)$ and porosity, $(\mathrm{Phi}, \%)$ listed for each core section are averages over $15-\mathrm{cm}$ intervals. Thus the values listed after $0.0 \mathrm{~cm}$ are averages for $0-15 \mathrm{~cm}$, and so forth. Eighty measurements are averaged for each $15-\mathrm{cm}$ interval. Data for each core section are summarized in a list of section averages of wet bulk density and porosity.

\section{ACKNOWLEDGMENTS}

We thank Ted Gustafson and his shipboard technical staff for making the routine GRAPE measurements and Tom Birtley of the DSDP staff for final editing and processing of the data.

\section{REFERENCE}

Boyce, R.E., 1976. Definitions and laboratory techniques of compressional sound velocity parameters and wet-water content, wet-bulk density, and porosity parameters by gravimetric and gamma ray attenuation techniques. In Schlanger, S.O., Jackson, E.D., et al., Initial Reports of the Deep Sea Drilling Project, v. 33: Washington (U.S. Government Printing Office), p. 931-958. 

for Core Sections Obtained During DSDP Leg 43

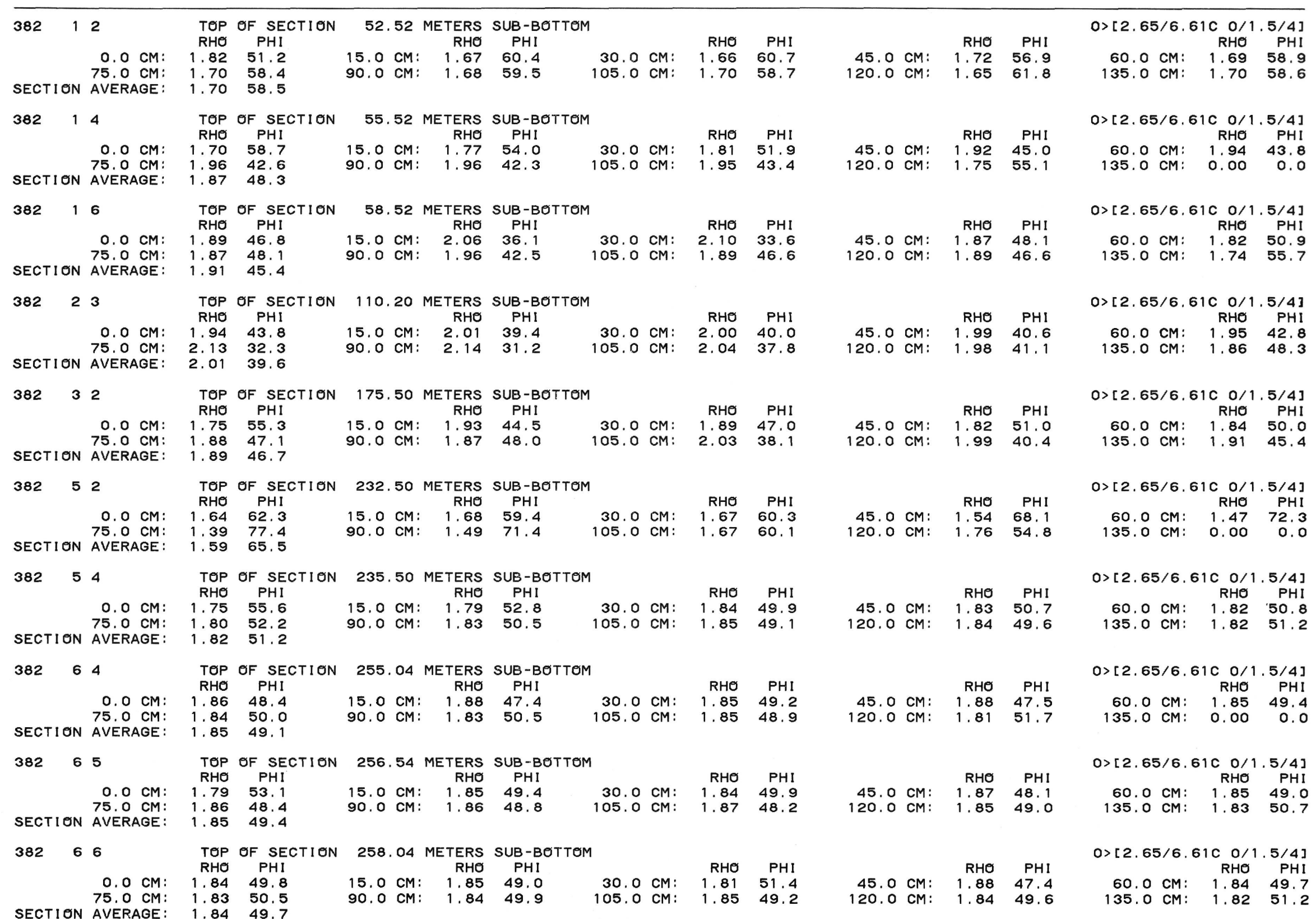


45. $\mathrm{CM}: \quad \begin{array}{rr}\mathrm{RHO} & \mathrm{PHI} \\ 1.81 & 51.6\end{array}$

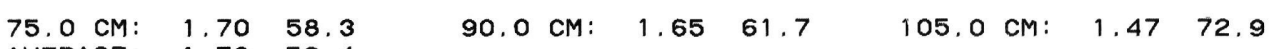
SECTION AVERAGE: $\quad 1.73 \quad 56.4$

38272

TOP OF SECTION 270.85 METERS SUB-BOTTOM

RHO PHI

0. $\mathrm{CM}: \quad 1.73 \quad 56.4$

SECTION AVERAGE: $1.76 \quad 54.7$

38273

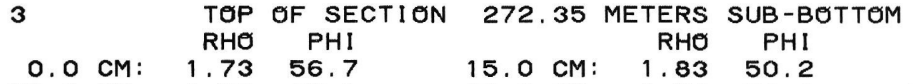

$\begin{array}{lllllll}\text { RHO } & \text { PHI }\end{array}$

SECTION AVERAGE: 1.7356 .8

38274

TOP OF SECTION 273.85 METERS SUB-BOTTOM

$\mathrm{RHO} \mathrm{PHI}$

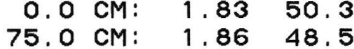

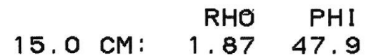

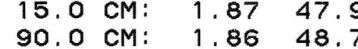

$\begin{array}{rrr}30.0 \mathrm{CM}: & \mathrm{RHO} & \mathrm{PHI} \\ 105.88 & 47.2\end{array}$

SECTION AVERAGE: $\quad 1.8748 .1$

38275

$\begin{array}{llll}\text { TOP OF SECTION 275.35 METERS SUB-BOTTOM } & \\ \text { RHO PHI } & \text { RHO PHI PHO PHI }\end{array}$ $0.0 \mathrm{CM}: \quad \begin{array}{r}\mathrm{RHO} \\ \mathrm{PHI}\end{array}$

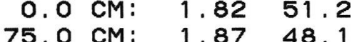

SECTION AVERAGE: $1.87 \quad 47.8$

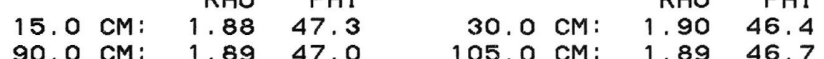

38276

TOP OF SECTION 276.85 METERS SUB-BOTTOM

$0.0 \mathrm{CM}: \quad \begin{array}{r}\mathrm{RHO} \\ \mathrm{PHI}\end{array}$

75.0 CM: 1.80 46.4

15.0 $\mathrm{RHO}$ PHI

$\begin{array}{llllll} & & & & \\ \text { RHO } & \text { PHI }\end{array}$

SECTION AVERAGE: 1.88 47.6

$\begin{array}{lll}382 & 8 & 1\end{array}$

TOP OF SECTION 279.04 METERS SUB-BOTTOM

RHO PHI

$0.0 \mathrm{CM}: 1.7754 .2$

$\begin{array}{lll}75.0 \mathrm{CM}: & 1.85 & 49.1 \\ \text { SECTION AVERAGE: } & 1.82 & 50.8\end{array}$

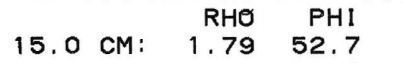

$382 \quad 82$

TOP OF SECTION 280.54 METERS SUB-BOTTOM

RHO PHI

O.0 CM: $\quad 1.75 \quad 55.4$

SECTION AVERAGE: 1.83 50.6

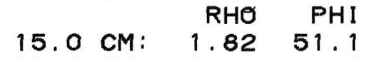

30. $0 \mathrm{CM}: \quad \mathrm{RHO} \mathrm{PHI}$

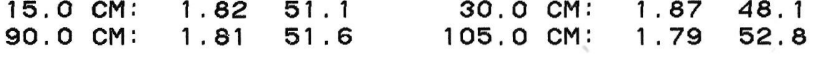

TOP OF SECTION 282.04 METERS SUB-BOTTOM

$382 \quad 83$

RHO PHI

0.0 CM: $1.74 \quad 56.0$

$15.0 \mathrm{cM}$ : RHO PHI

$\begin{array}{lll}15.0 \mathrm{CM}: & 1.79 & 52.9 \\ 90.0 \mathrm{CM}: & 1.85 & 49.4\end{array}$

$30.0 \mathrm{CM}: \quad \begin{array}{rrr}\mathrm{RHO} & \mathrm{PHI} \\ 1.83 & 50.4\end{array}$

SECTION AVERAGE: 1.8251 .4

$382 \quad 8 \quad 4$

TOP OF SECTION 283.54 METERS SUB-BOTTOM

$\begin{array}{rrr}0.0 \mathrm{CM}: & 1.79 & \mathbf{5 2 . 8} \\ 75.0 \mathrm{CM}: & 1.88 & 47.7\end{array}$

$15.0 \mathrm{cM}$ RHO PHI

15. O CM: $1.84 \quad 49.8$

$\begin{array}{rrr}\text { 30. O CM: } & \text { RHO } & \text { PHI } \\ 105.84 & 49.8\end{array}$
$120.0 \mathrm{CM}: 1.7655 .0$ $\begin{array}{rrr} & & \\ 120.0 \mathrm{CM}: & 1.69 & 59.2 \\ & 1.69 & 59.4\end{array}$

45.0 CM: $\begin{array}{rr}\text { RHO } & \text { PHI } \\ 1.62 & 63.3\end{array}$ 120.0 CM: 1.83 50.6

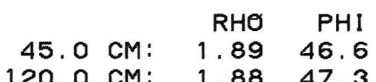
45.0 CM: $\quad 1.87$ 48.0

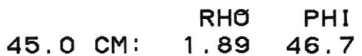
120.0 CM: 1.8946 .7

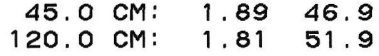
$\begin{array}{lll}20.0 \text { CM: } & 1.84 & 49.8 \\ & 1.83 & 50.3\end{array}$

45. 0 CM: RHO PHI $\begin{array}{rrr}45.0 \mathrm{CM}: & 1.85 & 49.5 \\ 120.0 \mathrm{CM}: & 1.85 & 49.0\end{array}$
45. O CM: $\begin{array}{rr}\mathrm{RHO} & \mathrm{PHI} \\ 1.69 & 59.2\end{array}$

45.0 CM: $\quad \begin{array}{rr}\mathrm{RHO} & \mathrm{PHI}\end{array}$

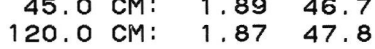

45. O CM: $\begin{array}{rr}\text { RHO } & \text { PHI } \\ 1.82 & 51.4\end{array}$

45.0 CM: $\quad \begin{array}{rr}\text { RHO } & \text { PHI } \\ 1.89 & 46.9\end{array}$

45.0 CM: $\begin{array}{rr}\text { RHO } & \mathrm{PHI} \\ 1.84 & 49.8\end{array}$
$0>[2.65 / 6.61 \mathrm{C} \quad 0 / 1.5 / 4]$

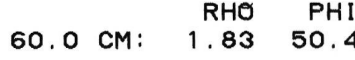

$135.0 \mathrm{CM}: \quad 1.77 \quad 54.2$

$0>[2.65 / 6.61 \mathrm{C} \quad 0 / 1.5 / 4]$ 60.0 CM: $\quad$\begin{tabular}{rr} 
RHO & PHI \\
\hline &
\end{tabular} $135.0 \mathrm{CM}: 1.7953 .2$

$0>[2.65 / 6.61 \mathrm{C} \quad 0 / 1.5 / 4]$ $60.0 \mathrm{cM}: \quad \begin{array}{rr}\mathrm{RHO} & \mathrm{PHI} \\ 1.46 & 73.5\end{array}$ 135.0 CM: $1.80 \quad 52.3$

$0>[2.65 / 6.61 \mathrm{C} 0 / 1.5 / 4]$ $60.0 \mathrm{CM}: \quad \begin{array}{rr}\mathrm{RHO} & \mathrm{PHI}\end{array}$ 135. O CM: 1.8549 .2

$0>[2.65 / 6.61 \mathrm{C} 0 / 1.5 / 4]$ $\begin{array}{llll}60.0 & \mathrm{CM}: & \mathrm{RHO} & \mathrm{PHI} \\ 1.86 & 48.7\end{array}$ $135.0 \mathrm{CM}: \quad 1.88 \quad 47.5$

$0>[2.65 / 6.61 \mathrm{C} 0 / 1.5 / 4]$ $60.0 \mathrm{~cm}: \quad \begin{array}{rr}\mathrm{RHO} & \mathrm{PHI}\end{array}$ $135.0 \mathrm{CM}: 1.88$ 47.2

$0>[2.65 / 6.61 \mathrm{C} \quad 0 / 1.5 / 4]$ $60.0 \mathrm{cM}: \quad \begin{array}{r}\mathrm{RHO} \\ \mathrm{PHI}\end{array}$ 135.0 CM: 1.8151 .9

$0>[2.65 / 6.61 \mathrm{C} 0 / 1.5 / 4]$ $60.0 \mathrm{CM}: \quad \begin{array}{rr}\mathrm{RHO} & \mathrm{PHI} \\ 1.86 & 48.3\end{array}$ 135.0 CM: 1.83 50.5

$0>[2.65 / 6.61 \mathrm{C} 0 / 1.5 / 4]$ $60.0 \mathrm{~cm}: \quad \begin{array}{r}\text { RHO } \\ 1.85\end{array}$ $\begin{array}{lll}1.74 & 55.8\end{array}$

$0>[2.65 / 6.61 \mathrm{C} 0 / 1.5 / 4]$ RHO PHI $135.0 \mathrm{cM}: 1.80 \quad 52.0$ 


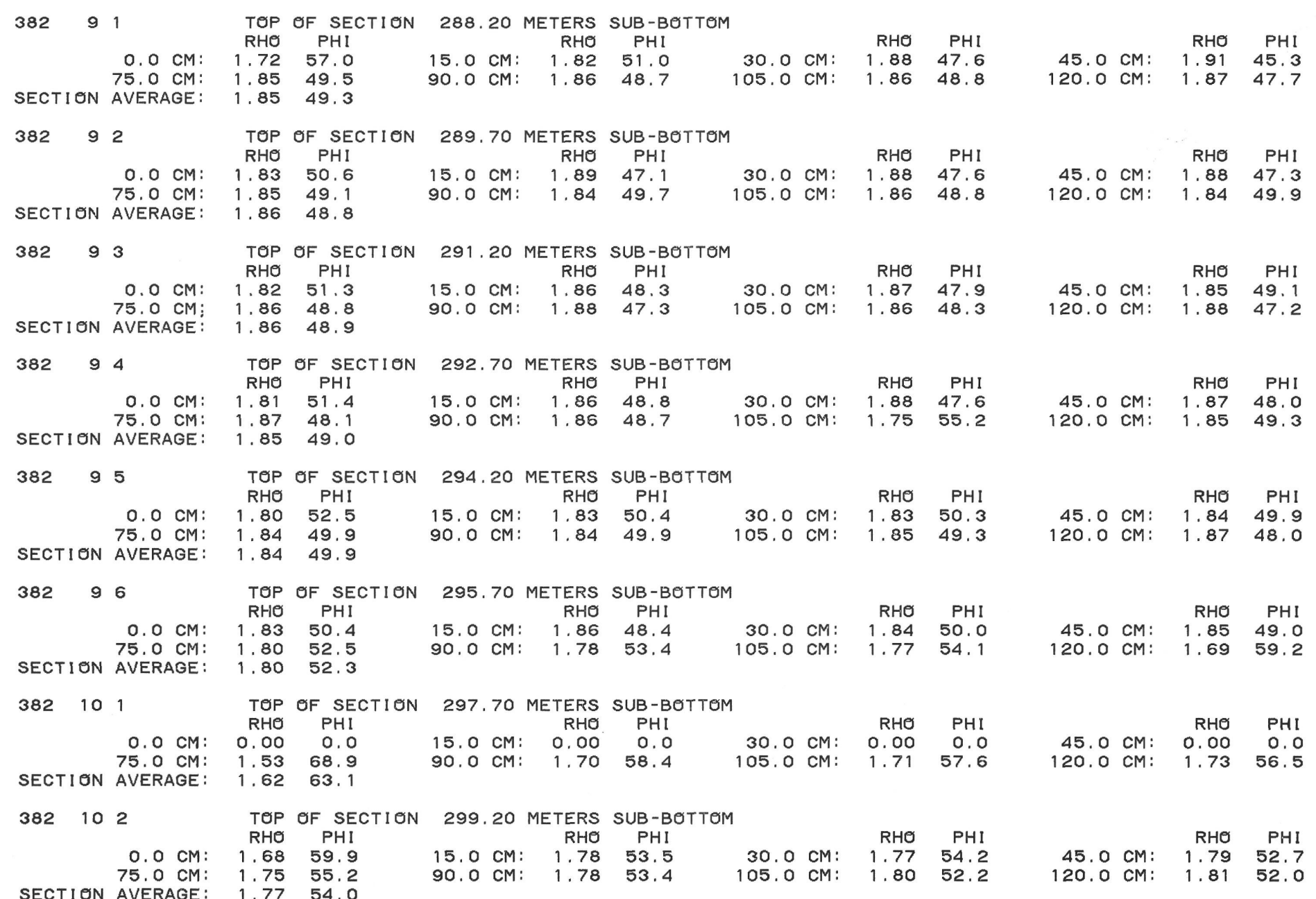

$0>[2.65 / 6.61 \mathrm{C} \quad 0 / 1.5 / 4]$ 60.0 CM: $\begin{array}{rr}\text { RHO } & \text { PHI } \\ 1.85 & 49.5\end{array}$ $135.0 \mathrm{CM}: \quad 1.88 \quad 47.2$

$0>[2.65 / 6.61 \mathrm{C} \quad 0 / 1.5 / 4]$ 60.0 CM: $\quad$ RHO PHI $135.0 \mathrm{cM}: 1.83$ 50.3

$0>[2.65 / 6.61 \mathrm{C} \quad 0 / 1.5 / 4]$

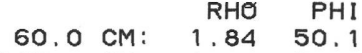
$135.0 \mathrm{CM}: 1.83$ 50.6

$0>[2.65 / 6.61 \mathrm{C} \quad 0 / 1.5 / 4]$ 60.0 cM: $\begin{array}{rrr}\text { RHO } & \text { PHI } \\ 1.86 & 48.7\end{array}$ $135.0 \mathrm{CM}: 1.91 \quad 45.3$

$0>[2.65 / 6.61 \mathrm{C} 0 / 1.5 / 4]$ $60.0 \mathrm{cM}$ RHO PHI 135.0 CM: 1.85 49.5

$0>[2.65 / 6.61 \mathrm{C} 0 / 1.5 / 4]$ $60.0 \mathrm{RH}: \mathrm{PHI}$ 135.0 CM: $1.71 \quad 57.7$

O) $[2.65 / 6.61 \mathrm{C} 0 / 1.5 / 4]$ $60.0 \mathrm{CM}: \quad \mathrm{RHO} \quad \mathrm{PHI}$ 135.0 CM: 1.63 62.8

$0>[2.65 / 6.61 \mathrm{C} 0 / 1.5 / 4]$ $60.0 \mathrm{cM}: \quad \begin{array}{r}\mathrm{RHO} \\ \mathrm{PHI}\end{array}$ $135.0 \mathrm{CM}: 1.78$ 53.3 
TOP OF SECTION 307.80 METERS SUB-BOTTOM

$\mathrm{RHO} \mathrm{PHI}$

0.0 CM: $1.78 \quad 53.6$

15. $\mathrm{cM}$ RHO $\quad$ PHI

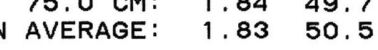

$\begin{array}{lll} & & \\ 90.0 \mathrm{CM}: & 1.83 & 50.4 \\ & & \end{array}$

30. $\mathrm{CM}$ : RHO PHI

TOP OF SECTION 309.30 METERS SUB-BOTTOM

$382 \quad 112$

$\begin{array}{rr}\text { RHO } & \mathrm{PHI} \\ 1.81 & 51.8\end{array}$

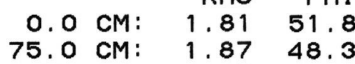

$\begin{array}{rrr} & \text { RHO } & \text { PHI } \\ 15.0 \mathrm{cM}: & 1.83 & 50.6\end{array}$

30.0 CM: $\begin{array}{rr}\text { RHO } & \text { PHI } \\ 1.85 & 49.1\end{array}$

$90.0 \mathrm{CM}: \quad 1.86 \quad 48.4$

105.0 CM: 1.86 48.5

SECTION AVERAGE: $1.85 \quad 49.4$

$382 \quad 113$

TOP OF SECTION 310.80 METERS SUB-BOTTOM

$\begin{array}{rrrr}0.0 & \mathrm{CM}: & \mathrm{RHO} & \mathrm{PH} \\ 75.0 \mathrm{CM}: & 1.89 & 53 . \\ & 1.86 & 48 .\end{array}$

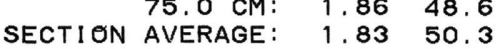

15.0 CM: $\quad \begin{array}{r}\text { RH } \\ 1.85\end{array}$

30.0 CM: $\quad \begin{array}{rr}\text { RHO } & \text { PHI } \\ 1.86 & 48.4\end{array}$

90.0 CM: 1.85 49.1

105.0 CM: $\quad 1.84$ 50.0

TOP OF SECTION 312.30 METERS SUB-BOTTOM

$382 \quad 11 \quad 4$

$\mathrm{RHO} \mathrm{PH}$

O. $0 \mathrm{CM}$

$\begin{array}{ll}1.79 & 52.9 \\ 1.84 & 49.6\end{array}$

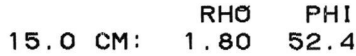

30.0 cM: $\quad$ RHO PHI

75. $0 \mathrm{CM}:$

SECTION AVERAGE:

TOP OF SECTION $\mathbf{3 1 3 . 8 0}$ METERS SUB-BOTTOM

$382 \quad 115$

O $\mathrm{CM}: \quad \mathrm{PHO} \mathrm{PHI} \quad \mathrm{RHO} \mathrm{PHI} \quad \mathrm{RHO} \mathrm{PHI}$

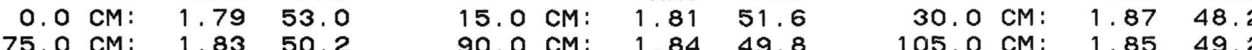

SECTION AVERAGE

$382 \quad 116$

TUP OF SECTION $\mathbf{3 1 5 . 3 0}$ METERS SUB-BOTTOM

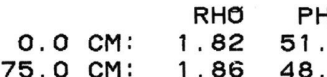

SECTION AVERAGE: $1.84 \quad 49.8$

RHO PHI

$\begin{array}{llllll}15.0 & \mathrm{CM}: & 1.85 & 49.3 & & \\ 90.0 \mathrm{CM}: & 1.85 & 49.0 & 105.0 \mathrm{CM}: & 1.85 & 49.4\end{array}$

TOP OF SECTION $\mathbf{3 1 7 . 3 0}$ METERS SUB-BOTTOM

382121

RHO PHI

O.O CM: $\quad 1.64 \quad 62.2$

$\begin{array}{ll}\text { RHO PHI } \\ 15.0 \mathrm{cM} & 1.74 \text { } 55.8\end{array}$

$\begin{array}{lll}75.0 \mathrm{CM}: & 1.74 & \mathbf{5 6 . 0} \\ \text { AVERAGE: } & 1.73 & \mathbf{5 6 . 7}\end{array}$

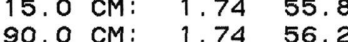

30. 0 : RHO 1.75 PHI

$\begin{array}{lll} & & \\ 05.0 \mathrm{CM}: & 1.75 & 55.5 \\ & & \end{array}$

SECTION AVERAGE:

$382 \quad 122$

TOP OF SECTION $\mathbf{3 1 8 . 8 0}$ METERS SUB-BOTTOM

$\mathrm{RHO} \quad \mathrm{PH}$

0.0 CM: $1.68 \quad 59.6$

$\begin{array}{rrr} & \text { RHO } & \text { PHI } \\ 15.0 \mathrm{cM}: & 1.73 & 56.3\end{array}$

RHO PHI

75.0 CM: SECTION AVERAGE

$382 \quad 123$

$\begin{array}{rrr}0.0 \mathrm{CM}: & \text { RHO } & \mathrm{PHI} \\ 75.66 & 61.1\end{array}$

TOP OF SECTION 320.30 METERS SUB-BOTTOM

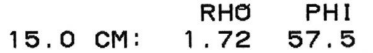

30.0 CM: $\quad \begin{array}{rrr}\text { RHO } & \text { PHI } \\ 1.72 & 57.3\end{array}$

SECTION AVERAGE: $1.72 \quad 57.1$

$382 \quad 124$

TOP OF SECTION 321.80 METERS SUB-BOTTOM

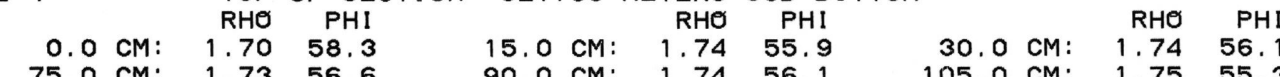

45. $\mathrm{CM}: \mathrm{RHO} \mathrm{PHI}$

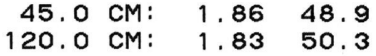

45. $\mathrm{CM}$ RHO PHI

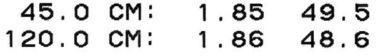

RHO PHI $\begin{array}{rrr}45.0 \mathrm{CM}: & 1.81 & 51.5 \\ 120.0 \mathrm{CM}: & 1.82 & 50.8\end{array}$

45. O CM: $\begin{array}{rr}\text { RHO } & \text { PHI } \\ 1.85 & 49.3\end{array}$ 120.0 CM: 1.84 49.8

45. O CM: $\quad$ RHO PHI 45. O CM: 1.86 48.9

RHO $\mathrm{PHI}$ 45. O CM: 1.84 49.9
120.0 CM: 1.85 49.1

45.0 CM: $\quad \begin{array}{r}\text { RHO } \\ \text { PHI }\end{array}$ 120.0 CM: 1.73 56.6

45. O CM: $\quad \begin{array}{rr}\text { RHO } & \text { PHI }\end{array}$ $\begin{array}{rrr}45.0 \mathrm{CM}: & 1.73 & \mathbf{5 6 . 5} \\ 120.0 \mathrm{CM}: & 1.72 & \mathbf{5 7 . 5}\end{array}$

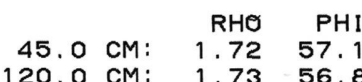

RHO PHI 45.0 CM: $1.73 \quad 56.8$
$0>[2.65 / 6.61 \mathrm{C} 0 / 1.5 / 4]$ $60.0 \mathrm{cM}: \quad \begin{array}{rr}\mathrm{RHO} & \mathrm{PHI}\end{array}$ $\begin{array}{lll}135.0 \mathrm{CM}: & 1.76 & 54.7\end{array}$

$0>[2.65 / 6.61 \mathrm{C} \quad 0 / 1.5 / 4]$

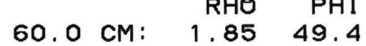
135.0 CM: 1.8549 .3

$0>[2.65 / 6.61 \mathrm{C} \quad 0 / 1.5 / 4]$

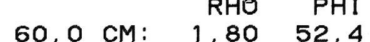
$135.0 \mathrm{CM}: \quad 1.84$ 49.8

$0>[2.65 / 6.61 \mathrm{C} \quad 0 / 1.5 / 4]$ 60.0 CM: $\begin{array}{rrr}\mathrm{RHO} & \mathrm{PHI} \\ & 1.87 & 48.0\end{array}$

$0>[2.65 / 6.61 \mathrm{C} \quad 0 / 1.5 / 4]$ $60.0 \mathrm{~cm}: \quad \begin{array}{rr}\mathrm{RHO} & \mathrm{PH} \\ 1.84 & 49.8\end{array}$

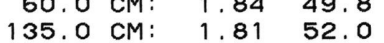

$0>[2.65 / 6.61 \mathrm{C} 0 / 1.5 / 4]$ 60.0 CM: $1.85 \quad 49.5$ $135.0 \mathrm{cM}: \quad 1.81 \quad 52.0$

$0>[2.65 / 6.61 \mathrm{C} 0 / 1.5 / 4]$ 60.0 CM: $\quad \begin{array}{rr}\text { RHO } & \text { PH.2 }\end{array}$ $135.0 \mathrm{CM}: 1.71 \quad 57.6$

$0>[2.65 / 6.61 \mathrm{C} 0 / 1.5 / 4]$ $60.0 \mathrm{cM}: \quad \begin{array}{rr}\mathrm{RHO} & \mathrm{PHI} \\ 1.73 & 56.6\end{array}$ $135.0 \mathrm{cM}: 1.71 \quad 57.7$

$0>[2.65 / 6.61 \mathrm{C} 0 / 1.5 / 4]$ $\begin{array}{rrr}60.0 \mathrm{CM}: & 1.73 & 56.7\end{array}$ $0>[2.65 / 6.61 \mathrm{C} 0 / 1.5 / 4]$

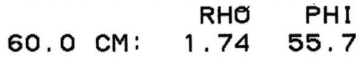
$135.0 \mathrm{CM}: \quad 0.00$ 0.0 


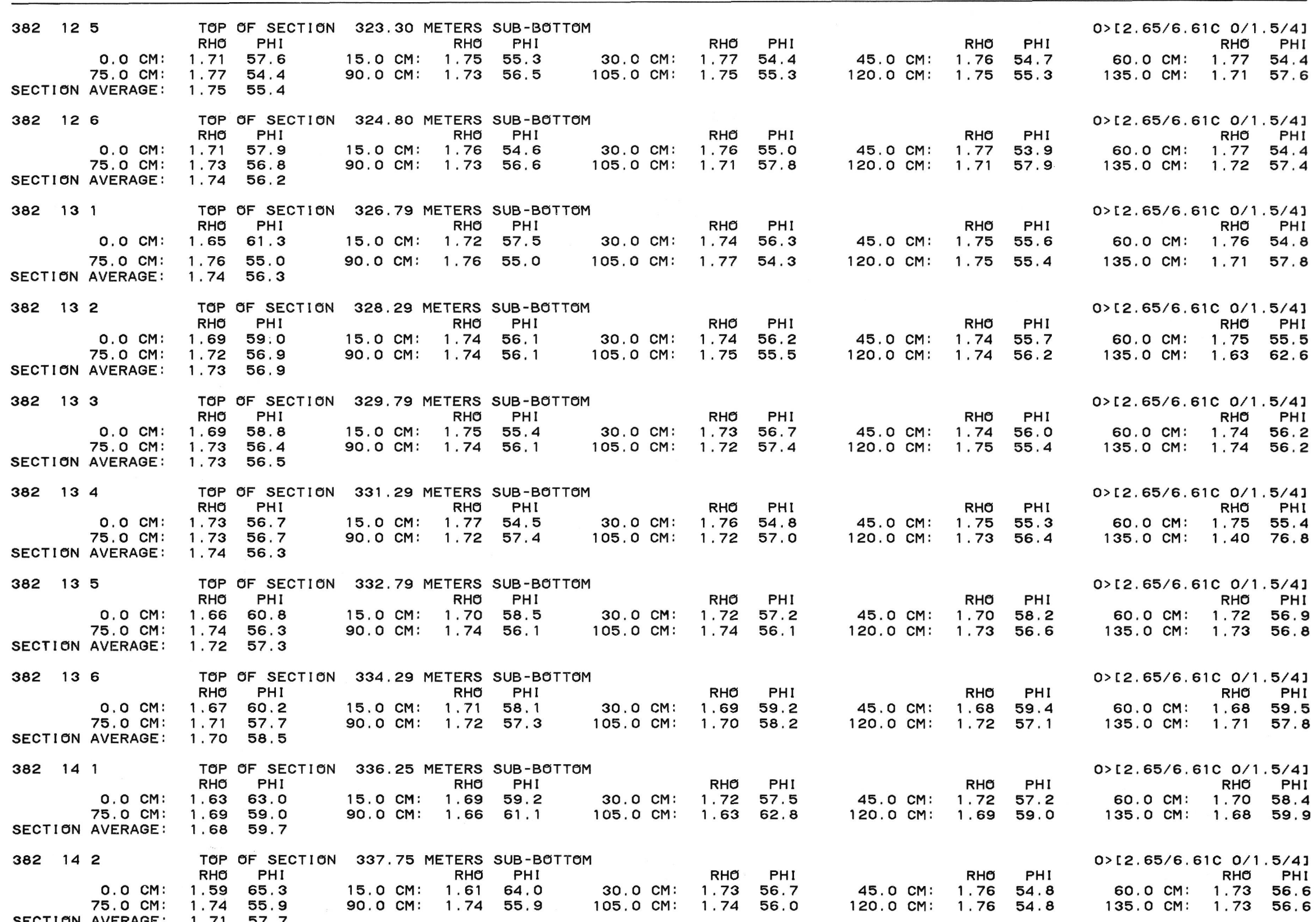


TOP OF SECTION 339.25 METERS SUB-BOTTOM

O CM: RHO PHI RHO PHI

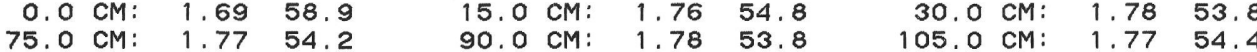

SECTION AVERAGE: $\quad 1,75 \quad 55.5$

$382 \quad 144$

\section{TOP OF SECTION 340.75 METERS SUB-BOTTOM}

O CM: RHO PHI RHO PHI RHO PHI

$75.0 \mathrm{CM}: 1.79 \quad 52.7$

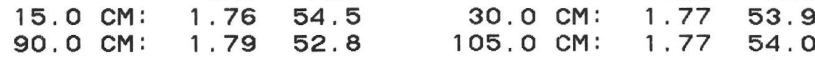

382145

TOP OF SECTION 342.25 METERS SUB-BOTTOM

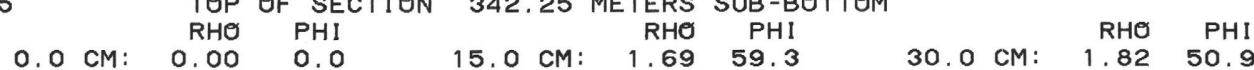

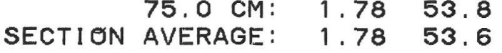

$382 \quad 146$

TOP OF SECTION 343.75 METERS SUB-BOTTOM

15.0 $\mathrm{CM}: \quad 1.80$ PHI

O.O CM: $\quad \begin{array}{rr}\text { RHO } & \text { PHI } \\ 75.0 & 54.9\end{array}$

15.0 CM: $1.80 \quad 52.2$

30.0 CM: $\quad 1.82 \quad 51.4$

$\begin{array}{ll}1.79 & 52.7 \\ 1.80 & 52.0\end{array}$

$38215 \quad 1$

TOP OF SECTION

O. O CM:
$75.0 \mathrm{CM}:$

SECTI ON AVERAGE:

382152

$0.0 \mathrm{CM}:$

$1.70 \quad 58.7$

$1.75 \quad 55.4$

RHO PHI

$30.02 \mathrm{RHO}^{2} \mathrm{PHI}$

90.0 CM: 1.77 54.1 $105.0 \mathrm{cM}: 1.76 \quad 54.6$

TOP OF SECTION 347.38 METERS SUB-BOTTOM

RHO PHI RHO PHI RHO PHI

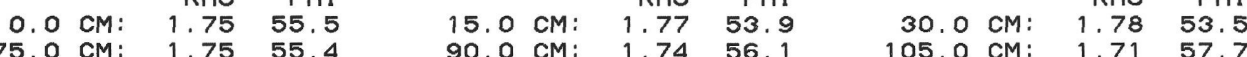

SECTION AVERAGE: $1.74 \quad 56.0$

$382 \quad 153$

TOP OF SECTION 348.88 METERS SUB-BOTTOM

$0.0 \mathrm{CM}:$

$1.70 \quad 58.3$

348.88 METERS SUB-BOTTOM

75. O CM: $\quad 1.77 \quad 54.0$

RHO PHI

$\begin{array}{lll}382 & 15 \quad 4\end{array}$

TOP OF SECTION $\mathbf{3 5 0 . 3 8}$ METERS SUB-BOTTOM

RHO PHI 15 RHO PHI RHO PHI

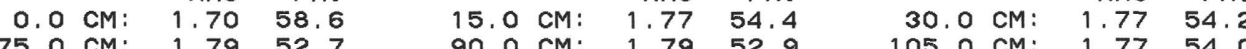

SECTION AVERAGE: $\quad 1.77 \quad 54.0$

$382 \quad 155$

TOP OF SECTION 351.88 METERS SUB-BOTTOM

RHO PHI RHO PHI

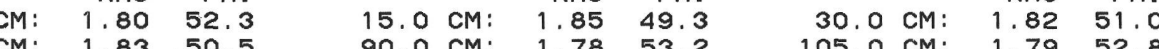

$\begin{array}{lll}75.0 \mathrm{CM}: & 1.83 & 50.5 \\ \text { SECTION AVERAGE: } & 1.81 & 51.5\end{array}$

382156

TOP OF SECTION 353.38 METERS SUB-BOTTOM

$\mathrm{RHO} \quad \mathrm{PHI}$

$0.0 \mathrm{CM}: \quad 1.81 \quad 52.0$

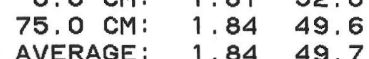

15.0 CM: 1.83 PHI $30.0 \mathrm{~cm}:$ RHO PHI

90.0 CM: 1.87 48.2 $105.0 \mathrm{cM}: 1.86 \quad 48.6$
45. $\mathrm{CM}: \quad \mathrm{RHO} \quad \mathrm{PHI}$ 120.0 CM: 1.71 57.

45. 0 CM: $\quad 1.77 \quad$ RHI $120.0 \mathrm{CM}: \quad 1.77 \quad 54.3$

45. O CM: $\quad \begin{array}{rr}\text { RHO } & \text { PHI }\end{array}$ $\begin{array}{rrr}45.0 \mathrm{CM}: & 1.81 & \mathbf{5 1 . 5} \\ 120.0 \mathrm{CM}: & 1.75 & \mathbf{5 5 . 6}\end{array}$

45. 0 cM: $\quad \begin{array}{r}\text { RHO } \\ \text { PHI }\end{array}$ $\begin{array}{rrr}45.0 \mathrm{CM}: & 1.83 & 50.3 \\ 120.0 \mathrm{CM}: & 1.81 & 51.4\end{array}$

RHO PHI

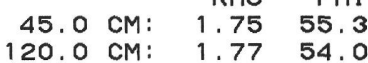

45.0 CM: $\begin{array}{rr}\text { RHO } & \text { PHI } \\ 1.74 & 55.7\end{array}$ $120.0 \mathrm{CM}: 1.7158 .7$

45.0 cM: $\begin{array}{rrr}\text { RHO } & \text { PHI } \\ 1.77 & 54.0\end{array}$ $120.0 \mathrm{CM}: \quad 1.77 \quad 53.9$

45. O cM: 1.78 PHI 45. C CM: 1.78 53.3

45. $\mathrm{CM}: \mathrm{RHO} \mathrm{PHI}$

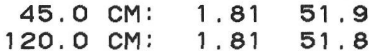

45. O CM: $\begin{array}{rr}\text { RHO } & \text { PHI } \\ 1.87 & 48.2\end{array}$ 120.0 CM: 1.84 50.0
$0>[2.65 / 6.61 \mathrm{C} \quad 0 / 1.5 / 4]$ 60.0 CM: 1.74 R5 135. O CM: $1.76 \quad 54.9$

$0>[2.65 / 6.61 \mathrm{C} \quad 0 / 1.5 / 4]$ 60.0 CM: $1.77 \quad 54.4$ 135.0 CM: $1.77 \quad 54.4$

$0>[2.65 / 6.61 \mathrm{C} \quad 0 / 1 \quad 5 / 4]$ 60.0 CM: $\begin{array}{rr}\text { RHO } & \text { PHI } \\ 1.71 & 57.7\end{array}$ 135.0 CM: $0.00 \quad 0.0$

$0>[2.65 / 6.61 \mathrm{C} \quad 0 / 1.5 / 4]$

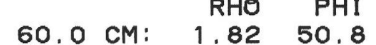
135.0 CM: 1.7754 .1

$0>[2.65 / 6.61 \mathrm{C} 0 / 1.5 / 4]$

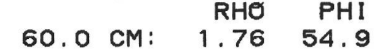
135.0 CM: 1.75 55.1

$0>[2.65 / 6.61 \mathrm{C} 0 / 1.5 / 4]$ 60.0 CM: $1.74 \quad 56.0$

$0>[2.65 / 6.61 \mathrm{C} \quad 0 / 1.5 / 4]$ RHO PHI

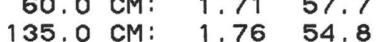

$0>[2.65 / 6.61 \mathrm{C} 0 / 1.5 / 4]$ $\begin{array}{rrr}60.0 \mathrm{cM}: & \text { RHO } & \mathrm{PHI} \\ 135.0 \mathrm{cM}: & 1.79 & \mathbf{5 3 . 2} \\ & \mathbf{5 4 . 3}\end{array}$

$0>[2.65 / 6.61 \mathrm{C} 0 / 1.5 / 4]$ 60.0 CM: $1.82 \quad 51.1$ $135.0 \mathrm{cM}: \quad 1.8251 .4$ $0>[2.65 / 6.61 \mathrm{C} \quad 0 / 1.5 / 4]$ $60.0 \mathrm{cM}: \quad \begin{array}{rr}\mathrm{RHO} & \mathrm{PHI} \\ 1.86 & 48.7\end{array}$ $135.0 \mathrm{CM}: 1.85$ 49.5 
$382 \quad 16$ 1 TOP OF SECTION 355.40 METERS SUB-BOTTOM RHO PHI $\quad$ RHO PHI $\quad$ RHO PHI

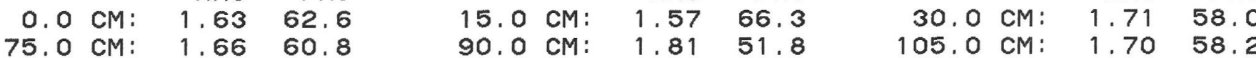
SECTION AVERAGE: .6859 .6

TOP OF SECTION 356.90 METERS SUB-BOTTOM

382162

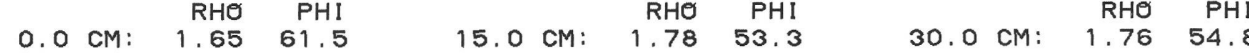

75.0 CM: 1.69 58.8 $\quad 90.0 \mathrm{CM}: 1.6064 .6 \quad 105.0 \mathrm{CM}: 1.6362 .8$

SECTION AVERAGE: 1.6958 .9

382163

TOP OF SECTION 358.40 METERS SUB-BOTTOM

$0.0 \mathrm{CM}: \quad \begin{array}{rr}\mathrm{RHO} & \mathrm{PHI} \\ 0.69 & 58.9\end{array}$

$75.0 \mathrm{CM}: 1.65$ 61.6

$\begin{array}{rrr} & \text { RHO } & \text { PHI } \\ 15.0 \mathrm{CM}: \quad 1.72 & 57.3\end{array}$

$\begin{array}{ll}1.65 & 61.6 \\ 1.67 & 60.1\end{array}$

$90.0 \mathrm{CM}: 1.66 \quad 60.7$

$105.0 \mathrm{CM}: \quad 1.6462 .1$

SECTION AVERAGE

TOP OF SECTION 359.90 METERS SUB-BOTTOM

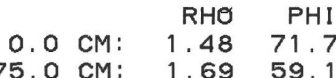

$\begin{array}{lll}75.0 \mathrm{CM}: & 1.69 & 59.1 \\ \text { AVERAGE: } & 1.63 & 62.8\end{array}$

$\begin{array}{lll}15.0 \mathrm{CM}: & \text { RHO } & \mathrm{PHI} \\ 1.61 & 64.0\end{array}$

30.0 $\mathrm{RHO} \mathrm{PHI}$

90.0 CM: 1.6163 .9

$\begin{array}{rrr}30.0 \mathrm{CM}: & 1.59 & 65.2 \\ 105.0 \mathrm{CM}: & 1.72 & 57.2\end{array}$

SECTION AVERAGE: $1.63 \quad 62.8$

382165

TOP OF SECTION $\mathbf{3 6 1 . 4 0}$ METERS SUB-BOTTOM

$\begin{array}{rrr}0.0 \mathrm{CM}: & 1.57 & 66.4 \\ 75.0 \mathrm{CM}: & 1.67 & 60.3\end{array}$

$\begin{array}{rrr} & \text { RHO } & \text { PHI } \\ 15.0 \mathrm{CM}: \quad 1.58 & 65.7\end{array}$

90. $\mathrm{CCM}: \quad 1.70$ 58.3

30. $0 \mathrm{CM}: \quad \begin{array}{rr}\mathrm{RHO} & \mathrm{PHI} \\ 1.61 & 63.9\end{array}$

$105.0 \mathrm{CM}: \quad 1.66$ 60.7

SECTION AVERAGE: $1.66 \quad 61.1$

TOP OF SECTION 362.90 METERS SUB-BOTTOM

RHO PHI RHO PHI RHO PHI

$0.0 \mathrm{CM}: \quad 1.45 \quad 73.7$

75.0 CM: 1.7555 .2

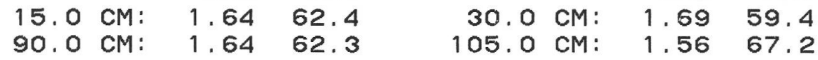

SECTION AVERAGE:

TOP OF SECTION 364.40 METERS SUB-BOTTOM

$382 \quad 17 \quad 1$

$0.0 \mathrm{CM}: 0.00 \mathrm{PHI}$

$\begin{array}{lll}75.0 \mathrm{CM}: & 1.57 & 66.5 \\ \text { AVERAGE : } & 1.62 & 63.4\end{array}$

$\begin{array}{lll}15.0 \mathrm{cM}: & \mathrm{RHO} & \mathrm{PHI} \\ 0.00 & 0.0\end{array}$

30.0 $\mathrm{RHO} \mathrm{PHI}$

SECTION AVE

TOP OF SECTION 365.90 METERS SUB-BOTTOM

$0.0 \mathrm{CM}: \begin{array}{rr}\mathrm{RHO} & \mathrm{PHI} \\ 1.53 & 69.2\end{array}$

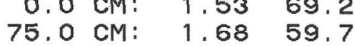

15.0 cM: 1.66 61.0

90.0 CM: 1.78 53.7

$30.0 \mathrm{CM}: \mathrm{RHO} \mathrm{PHI}$

1.6958 .9

$\begin{array}{lll}382 & 17 & 3\end{array}$

TOP OF SECTION 367.40 METERS SUB-BOTTOM

O CM: RHO PHI RHO PHI RHO PHI

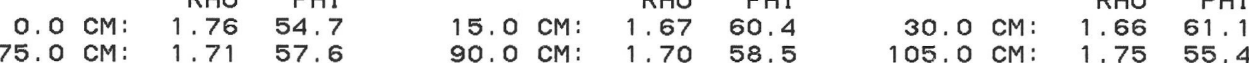

SECTION AVERAGE: $1.73 \quad 56.5$

TOP OF SECTION $\mathbf{3 6 8 . 9 0}$ METERS SUB-BOTTOM

$\begin{array}{lll}382 & 17 & 4\end{array}$

RHO PHI

$0.0 \mathrm{CM}: \quad 1.65 \quad 61.7$

SECTION AVERAGE: $\quad 1.71 \quad 57.8$

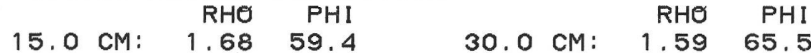
$90.0 \mathrm{CM}: 1.67$ 60.3 $105.0 \mathrm{CM}: 1.7754 .1$
45. O CM: RHO PHI 120.0 CM: 1.7456.

45. CM: 1.73 PHI $120.0 \mathrm{CM}: \quad 1.6462 .4$

45.0 CM: $\begin{array}{rr}\text { RHO } & \text { PHI } \\ 1.70 & 58.3\end{array}$ $\begin{array}{lll}120.0 \mathrm{CM}: & 1.70 & 58.3 \\ & 1.66 & 60.7\end{array}$

$\begin{array}{rrr}45.0 \mathrm{cM}: & \text { RHO } & \text { PHI } \\ 1.62 & 63.4\end{array}$ $\begin{array}{rrr}45.0 \text { CM: } & 1.62 & 63.4 \\ 120.0 \text { CM: } & 1.67 & 60.3\end{array}$ (1)

45. $0 \mathrm{cM}: \quad \mathrm{RHO} P \mathrm{PH}$ $\begin{array}{lll} & \\ 120.0 \mathrm{CM}: & 1.79 & 52.6\end{array}$

2HO PHI $\begin{array}{rrr}\text { 120.0 CM: } & 1.55 & 67.7 \\ & & \end{array}$

45. $\mathrm{CM}$ RHO PHI

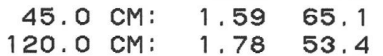

45.0 CM: RHO PHI

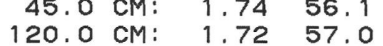

45. $\mathrm{RHO} \mathrm{PHI}$

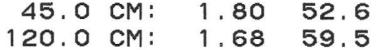

45. $\mathrm{CM}: \quad \mathrm{RHO} \mathrm{PHI}$ $\begin{array}{lll} & & \\ 120.0 \mathrm{CM}: & 1.70 & 58.8 \\ & & \end{array}$
$0>[2.65 / 6.61 \mathrm{C} 0 / 1,5 / 4]$ 60.0 CM: 1.64 62.4 $0>[2.65 / 6.61 \mathrm{C} 0 / 1,5 / 4]$ 60.0 cM: $\quad 1.77 \quad 54$. 135.0 CM: 1.67 60.2

$0>[2.65 / 6.61 \mathrm{C} 0 / 1.5 / 4]$ 60.0 CM: $\begin{array}{rr}\mathrm{RHO} & \mathrm{PHI} \\ 1.67 & 60.6\end{array}$ $135.0 \mathrm{CM}: 1.5965 .3$

$0>[2.65 / 6.61 \mathrm{C} 0 / 1.5 / 4]$ $60.0 \mathrm{cM}: \quad \begin{array}{rr}\mathrm{RHO} & \mathrm{PHI} \\ 1.62 & 63.4\end{array}$ $\begin{array}{lll}35.0 \mathrm{CM}: & 1.67 & 60.1\end{array}$

$0>[2.65 / 6.61 \mathrm{C} \quad 0 / 1.5 / 4]$ 60.0 CM: RHO PHI 135.0 CM: 1.7257 .1

$0>[2.65 / 6.61 \mathrm{C} 0 / 1.5 / 4]$

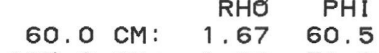
$135.0 \mathrm{cM}: 1.50 \quad 71.0$

$0>[2.65 / 6.61 \mathrm{C} 0 / 1.5 / 4]$ $60.0 \mathrm{cM}: \quad \begin{array}{rr}\text { RHO } & \mathrm{PHI} \\ 1.63 & 62.9\end{array}$ $135.0 \mathrm{CM}: 1.78 \quad 53.7$

$0>[2.65 / 6,61 \mathrm{C} 0 / 1.5 / 4]$ $60.0 \mathrm{cM}: \quad \begin{array}{rr}\mathrm{RHO} & \mathrm{PHI} \\ 1.76 & 54.6\end{array}$ 135.0 CM: 1.67 60.6

$0>[2.65 / 6.61 \mathrm{C} 0 / 1.5 / 4]$ 60.0 CM: $1.81 \quad 51.6$ 135.0 CM: 1.7952 .8

$0>[2.65 / 6.61 \mathrm{C} 0 / 1.5 / 4]$

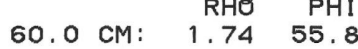
$135.0 \mathrm{cM}: \quad 1.72 \quad 57.0$ 
SUB-BOTTOM

75.0 CM: 1.63 63.7

$15.0 \mathrm{cM}: \quad 1.65 \quad 61.5$

90.0 CM: 1.7455 .8

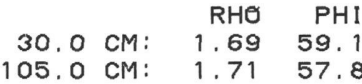

1.6663 .0

45. O CM: RHO PH $120.0 \mathrm{CM}: \quad 1.7158$.

$382 \quad 176$

$\begin{array}{rrr}0.0 \mathrm{CM}: & \text { RHO } & \mathrm{PHI} \\ 7.61 & 63.9\end{array}$

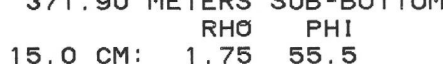

75.0 CM: 1.66 60.8

SECTION AVERAGE

$1.62 \quad 63.4$

$382 \quad 181$

TOP OF SECTION 373.80 METERS SUB-BOTTOM

RHO PHI RHO PHI RHO PHI $\begin{array}{rrr}0.0 \mathrm{CM}: & 0.00 & \text { PHI } \\ 75.0 \mathrm{CM}: & 1.62 & 63.1\end{array}$ 15.0 CM: $0.00 \quad 0.0$ 30.0 CM: 1.26 85.3 $\begin{array}{lll}\text { 75. O CM: } & 1.62 & 63.1 \\ \text { AVERAGE: } & 1.64 & 62.1\end{array}$ $90.0 \mathrm{CM}: \quad 1.73 \quad 56.8$

TOP OF SECTION 375.30 METERS SUB-BOTTOM

$382 \quad 182$

RHO PHI

$\begin{array}{rrr}15.0 \mathrm{CM}: & \mathrm{RHO} & \mathrm{PHI} \\ 1.78 & 53.7\end{array}$

30.0 CM: $\begin{array}{rr}\text { RHO } & \text { PHI } \\ 1.74 & 56.3\end{array}$

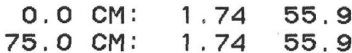

90.0 CM: 1.58 . 65.5

105.0 CM: 1.67 60.1

SECTION AVERAGE: 1.6760 .4

$382 \quad 18 \quad 3$

TOP OF SECTION 376.80 METERS SUB-BOTTOM

$$
0.0 \mathrm{~cm} \text { : }
$$

$\mathrm{RHO}$

15.0 CM: 1.62 PHI

30.0 CM: $\quad$ RHO $\mathrm{PHI}$

$1.70 \quad 58.4$

$90.0 \mathrm{CM}: \quad 1.5369 .2$

105.0 CM: $1.72 \quad 57.0$

SECTI ON AVERAGE

TOP OF SECTION 378.30 METERS SUB-BOTTOM

$382 \quad 18 \quad 4$

RHO PHI

$0.0 \mathrm{CM}: \quad 1.63 \quad 62.7$

15.0 cM: $\quad$ RHO PHI

30. $\mathrm{CM}: \quad \mathrm{RHO} \quad \mathrm{PHI}$

.6561 .3

$90.0 \mathrm{CM}: \quad 1.6362 .7$

30.0 CM: $1.70 \quad 58.2$

SECTION AVERAGE:

TOP OF SECTION 384.80 METERS SUB-BOTTOM

382192

$0.0 \mathrm{CM}: \quad 1.39 \quad 77.5$

RHE PHI

15.0 CM: $\quad \begin{array}{rr}\text { RHE } & 67.3 \\ & \end{array}$

$30.0 \mathrm{CM}: \quad \mathrm{RHO} \mathrm{PHI}$

75.0 CM: $\quad 1.92 \quad 45.1$

105. $0 \mathrm{CM}: 1.75 \quad 55.3$

$382 \quad 193$

TOP OF SECTION 386.30 METERS SUB-BOTTOM

RHO PHI

$0.0 \mathrm{CM}: \quad 1.83 \quad 50.4$

15.0 CM: RHO PHI

30. $\mathrm{CM}:$ RHO PHI

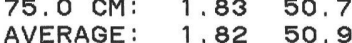

90.0 CM: 1.78 53.4

$105.0 \mathrm{CM}: 1.79 \quad 53.0$

$382 \quad 194$

TOP OF SECTION 387.80 METERS SUB-BఠTTOM

$\begin{array}{rr}\text { RHO } & \mathrm{PHI} \\ 1.94 & 43.7\end{array}$

O.O CM: $1.94 \quad 43.7$

SECTION AVERAGE: $1.82 \quad 51.0$

RHO PHI

30. $\mathrm{CM}: \quad \mathrm{RHO} \quad \mathrm{PHI}$

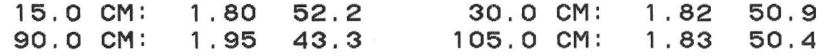

$\begin{array}{lll}382 & 20 & 1\end{array}$

TOP OF SECTION 402.30 METERS SUB-BOTTOM

$0.0 \mathrm{CM}: \mathrm{RHO} \mathrm{PHI}$

75.0 CM: $0.00 \quad 0.0$

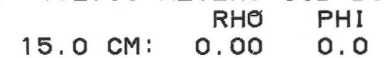

30.0 $\mathrm{CM}: \mathrm{PHI}$

SECTION AVERAGE: $1.77 \quad 54.0$

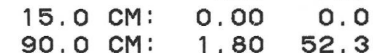
$105.0 \mathrm{CM}$

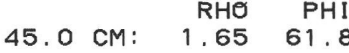
$120.0 \mathrm{CM}: 1.57$ 66.5

45. $\mathrm{cM}: \quad \begin{array}{r}\mathrm{RHO} \\ \mathrm{PHI}\end{array}$ $\begin{array}{rrr}45.0 \mathrm{CM}: & 1.67 & 60.5 \\ 120.0 \mathrm{CM}: & 1.62 & 63.4\end{array}$

RHO PHI

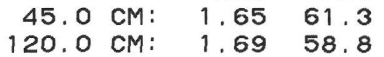$$
\begin{array}{rrr}
\text { 45. O CM: } & \text { RHO } & \text { PHI } \\
1.72 & 57.4 \\
120.0 \mathrm{CM}: & 1.76 & 55.0
\end{array}
$$

RHO PHI $\begin{array}{rrr}45.0 \mathrm{CM}: & 1.65 & 61.3 \\ 120.0 \mathrm{CM}: & 1.67 & 60.3\end{array}$

45.0 CM: $\quad \begin{array}{rrr}\text { RHO } & \text { PHI } \\ 1.51 & 69.9\end{array}$ $120.0 \mathrm{CM}: 1.82 \quad 51.1$

45. $\mathrm{CM}: \quad \mathrm{RHO} \quad \mathrm{PHI}$

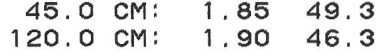

$45.0 \mathrm{~cm}$ RHO PHI

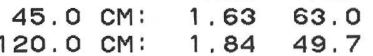

45.0 CM: $\begin{array}{rr}\text { RHO } & \text { PHI } \\ 0.00 & 0.0\end{array}$
$0>[2.65 / 6.61 \mathrm{C} 0 / 1.5 / 4]$ 60.0 cM: 1.59 PHI $135.0 \mathrm{CM}: \quad 1.6362 .9$

$0>[2.65 / 6.61 \mathrm{C} \quad 0 / 1.5 / 4]$ $60.0 \mathrm{~cm}: \quad \begin{array}{r}\mathrm{RHO} \\ \mathrm{PHI}\end{array}$ $135.0 \mathrm{CM}: 1.5070 .8$

$0>[2.65 / 6.61 \mathrm{C} 0 / 1.5 / 4]$ $60.0 \mathrm{cM}: \quad \begin{array}{rr}\mathrm{RHO} & \mathrm{PHI} \\ 1.56 & 67.2\end{array}$ 135.0 CM: 1.6561 .6

$0>[2.65 / 6.61 \mathrm{C} 0 / 1.5 / 4]$ $60.0 \mathrm{cM}: \quad \begin{array}{r}\mathrm{RHO} \\ 1.54 \\ 68.6\end{array}$ $135.0 \mathrm{CM}: \quad 1.5368 .7$

$0>[2.65 / 6.61 \mathrm{C} 0 / 1.5 / 4]$ $60.0 \mathrm{cM}: \quad \begin{array}{rr}\mathrm{RHO} & \mathrm{PHI} \\ 1.68 & 59.6\end{array}$ $135.0 \mathrm{CM}: \quad 1.3580 .2$

$0>[2.65 / 6.61 \mathrm{C} \quad 0 / 1.5 / 4]$ $60.0 \mathrm{cM}: \quad \mathrm{RHO} P \mathrm{PHI}$ 135.0 CM: 1.58 66.1

$0>[2.65 / 6.61 \mathrm{C} 0 / 1.5 / 4]$ 60.0 CM: $1.91 \quad 45.7$ $135.0 \mathrm{CM}: 1.6959 .3$

$0>[2.65 / 6.61 \mathrm{C} 0 / 1.5 / 4]$

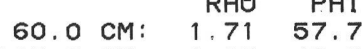
$135.0 \mathrm{CM}: \quad 1.85 \quad 48.9$

$0>[2.65 / 6.61 \mathrm{C} 0 / 1.5 / 4]$ $60.0 \mathrm{~cm}: \quad \begin{array}{rr}\mathrm{RHO} & \mathrm{PHI} \\ 1.69 & 58.8\end{array}$ 135.0 CM: $1.86 \quad 48.4$

$0>[2.65 / 6.61 \mathrm{C} 0 / 1.5 / 4]$

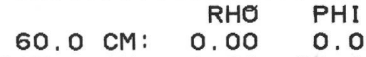
$135.0 \mathrm{CM}: \quad 1.6959 .3$ 
$38220 \quad 4$

\section{TOP OF SECTION 406.80 METERS SUB-BOTTOM}

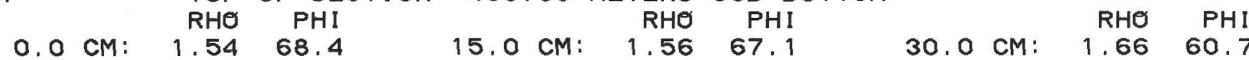

75.O CM: $1.49 \quad 71.2$

382205

\begin{tabular}{rrr} 
0.0 CM: $\quad 1.50$ & PHO & PHI \\
\hline & 1.8
\end{tabular}

90. 0 CM: 1.5170 .2

105.0 CM: $\quad 1.66 \quad 60.7$

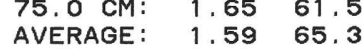

$382 \quad 21 \quad 1$

TOP OF SECTION 411.70 METERS SUB-BOTTOM

RHO PHI

$\begin{array}{rrr}0.0 \mathrm{CM}: & 0.00 & \mathrm{RH} \\ 7.0 & \end{array}$

$15.0 \mathrm{CM}: \quad 0.00 \quad 0.0$

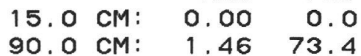

30.0 CM: 0.00 RHO

AVERAGE: $1.42 \quad 75.9$

TOP OF SECTION 413.20 METERS SUB-BOTTOM

$382 \quad 212$

RHO PHI

O. $\mathrm{CM}: \quad 1.46 \quad 73.2$

RHO PHI

30.0 cM: $162 \quad \mathrm{PHI}$

$\begin{array}{ll}.64 & 61.9 \\ .57 & 66.5\end{array}$

90.0 CM: $1.61 \quad 63.7$

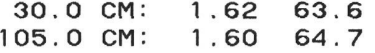

SECTION AVERAGE

TOP OF SECTION

$382 \quad 213$

$\begin{array}{rrr}0.0 \mathrm{CM}: & \begin{array}{r}\text { RHO } \\ 1.57\end{array} \quad 66.7\end{array}$

$75.0 \mathrm{CM}: 1.6362 .8$

15.0 cM: 1.75 PHO

RHO PHI

$\begin{array}{ll}.63 & 62.8 \\ .62 & 63.5\end{array}$

$90.0 \mathrm{CM}: 1.6561 .3$

105.0 CM: $1.68 \quad 59.5$

TOP OF SECTION 441.80 METERS SUB-BOTTOM

38222

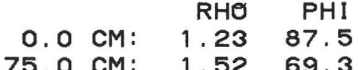
$\begin{array}{rrr}15.0 \mathrm{CM}: & \text { RHఠ } & \text { PHI } \\ 90.0 \mathrm{CM}: & 1.53 & 85.3 \\ 98.8\end{array}$ 30.0 CM: $\begin{array}{rr}\text { RHO } & \text { PHI } \\ 1.48 & 71.8\end{array}$ $75.0 \mathrm{CM}: 1.5269 .3$ SECTION AVERAGE

\section{TOP OF SECTION 443.30 METERS SUB-BOTTOM}

382223

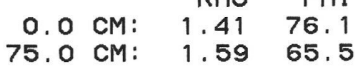

$15.0 \mathrm{~cm}: \quad \begin{array}{r}\mathrm{RHO} \\ \mathrm{PHI}\end{array}$

1.5965 .5

90. $\mathrm{CM}: \quad 1.48 \quad 72.2$

105.0 CM: $1.56 \quad 66.9$

SECTION AVERAGE:

TOP OF SECTION 471.90 METERS SUB-BOTTOM

$382 \quad 23 \quad 3$

O.O CM: $1.63 \quad 62.7$

SECTION AVERAGE: 1.6263 .6

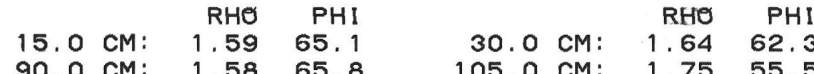

45.0 CM: $\quad \begin{array}{rrr}\mathrm{RHO} & \mathrm{PHI} \\ 1.53 & 68.8\end{array}$ $120.0 \mathrm{CM}: \quad 1.3281 .6$

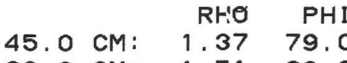
$120.0 \mathrm{CM}: \quad 1.5169 .9$

45. $\mathrm{CM}:$ RHO $\mathrm{PH}$ $120.0 \mathrm{CM}: \quad 1.4971 .4$

45. 0 CM: $\quad \begin{array}{r}\text { RHO } \\ 1.60\end{array}$ 120.0 CM: 1.6561 .8

45. O CM: $\quad \begin{array}{rrr}\text { RHO } & \text { PHI } \\ 74.44 & \end{array}$ 120.0 CM: $1.42 \quad 75.7$

15HO $\mathrm{PHI}$

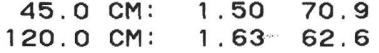

RHO PHI

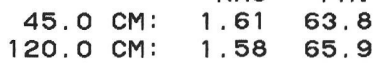

15HO PHI $\begin{array}{rrr}45.0 \mathrm{CM}: & 1.50 & 70.6 \\ 120.0 \mathrm{CM}: & 1.56 & 67.4\end{array}$

$45.0 \mathrm{~cm}: \quad \mathrm{RHO} \quad \mathrm{PH}$

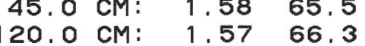

45. O CM: 1.53 PHI

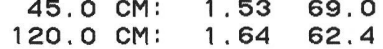

$0>[2,65 / 6,61 \mathrm{C} 0 / 1,5 / 4]$

$60.0 \mathrm{cM}: 1.66 \quad 60.8$ $135.0 \mathrm{cM}: 1.3580 .1$

$0>[2.65 / 6.61 \mathrm{C} 0 / 1.5 / 4]$ $60.0 \mathrm{cM}: \quad \begin{array}{r}\mathrm{RHO} \\ 1.50\end{array}$ $135.0 \mathrm{cM}: \quad 1.4474 .7$

$0>[2.65 / 6.61 C 0 / 1.5 / 4]$ $60.0 \mathrm{~cm}: \quad \begin{array}{rr}\mathrm{RHO} & \mathrm{PHI}\end{array}$ 135.0 CM: 1.56 67.1

$0>[2.65 / 6.61 \mathrm{C} \quad 0 / 1.5 / 4]$ $60.0 \mathrm{cM}: \quad 1.60$ PH. 135.0 CM: 1.5567 .5

$0>[2.65 / 6.61 \mathrm{C} 0 / 1.5 / 4]$ $60.0 \mathrm{~cm}: \quad \begin{array}{rr}\mathrm{RHO} & \mathrm{PHI} \\ 1.31 & 82.3\end{array}$ $135.0 \mathrm{CM}: 1.3977 .3$

$0>[2.65 / 6.61 \mathrm{C} 0 / 1.5 / 4]$ $60.0 \mathrm{~cm}: \quad \begin{array}{rr}\mathrm{RHO} & \mathrm{PHI} \\ 1.53 & 69.2\end{array}$ $135.0 \mathrm{CM}: \quad 1.6263 .4$

$0>[2.65 / 6.61 \mathrm{C} 0 / 1.5 / 4]$ 60.0 cM: $\begin{array}{rr}\text { RHO } & \text { PHI } \\ 1.55 & 67.6\end{array}$ 135.0 CM: $1.48 \quad 72.0$

$0>[2.65 / 6.61 \mathrm{C} 0 / 1.5 / 4]$ $\begin{array}{rrr}60.0 \mathrm{cM}: & \mathrm{RHO} & \mathrm{PHI} \\ 1.47 & 72.7\end{array}$ $35.0 \mathrm{CM}: 1.5667 .0$

$0>[2.65 / 6.61 \mathrm{C} 0 / 1.5 / 4]$ $60.0 \mathrm{~cm}: \quad \begin{array}{r}\mathrm{RHO} \\ 1.62\end{array}$ $135.0 \mathrm{CM}: 1.5865 .9$

$0>[2.65 / 6.61 \mathrm{C} 0 / 1.5 / 4]$ $60.0 \mathrm{cM}: \quad \begin{array}{r}\mathrm{RHO} \\ \mathrm{PHI}\end{array}$ $135.0 \mathrm{CM}: 1.6859 .9$ 
$383 \quad 13$

TH SECTION

O. $0 \mathrm{CM}:$

$75.0 \mathrm{CM}$

SECTION AVERAGE:

$384 \quad 1 \quad 1$

$$
\text { O.O CM: }
$$$$
2.22 \quad 26.2
$$

56.70 METERS SUB-BOTTOM
RHO PHI

15.0 CM: $\begin{array}{rrr}\text { RHO } & \text { PHI } \\ & 2.15 & 30.8\end{array}$

90.0 CM: $2.23 \quad 26.0$

30. $\mathrm{CM}: 2 \mathrm{RHO} \mathrm{PHI}$ 105.0 CM: 2.23 26.0

TOP OF SECTION 50.80 METERS SUB-BOTTOM

$0.00 \quad 0.0$

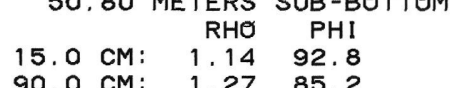

30. $\mathrm{CM}: \quad \mathrm{RHO} \mathrm{PHI}$

$1.26 \quad 85.5$

TOP OF SECTION 52.30 METERS SUB-BOTTOM

$384 \quad 12$

RHO PHI
RHO METERS SUB-BOTTOM

$0.0 \mathrm{CM}: \quad 1.28 \quad 84.0$

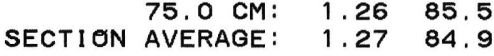

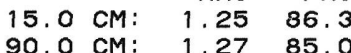

30.0 CM: $\quad \begin{array}{rr}\mathrm{RHO} & \mathrm{PHI} \\ 1.25 & 86.2\end{array}$

$384 \quad 13$

TOP OF SECTION

0.0 CM: RHO PHI

$\begin{array}{lll}0.0 & & \\ \text { CM: } & 1.24 & 86.8 \\ \text { CM: } & 1.28 & 84.2\end{array}$

53.80 METERS SUB-BOTTOM

SECTION AVERAGE: $\begin{array}{lll}1.28 & 84.6\end{array}$

$15.0 \mathrm{CM}$ RHO $\mathrm{PH}$

30.0 CM: $\quad$ RHO PHI

90.0 CM: 1.29 83.7

105.0 CM: 1.2983 .5

$384 \quad 44$

TOP OF SECTION

RHO PHI

$0.0 \mathrm{CM}: 1.3480 .7$

SECTION AVERAGE: 1.3381 .3

84.40 METERS SUB-BOTTOM

$\begin{array}{rrr}15.0 \mathrm{cM}: & \begin{array}{r}\text { RHO } \\ 1.33\end{array} \quad 81.0\end{array}$

30.0 CM: $\begin{array}{rr}\text { RHO } & \text { PHI } \\ 1.31 & 82.3\end{array}$ 90.0 CM: 1.34 80.9

105.0 CM: 1.3281 .6

TOP OF SECTION

85.90 METERS SUB-BOTTOM

$\begin{array}{rrrr}0.0 \mathrm{CM}: & 1.32 & 82.2 \\ 75.0 \mathrm{CM}: & 1.36 & 79.3\end{array}$

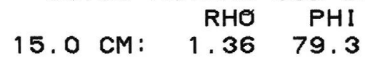

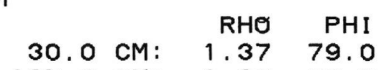

90. $0 \mathrm{CM}: 1.37 \quad 78.5$

105.0 CM: 1.3679 .3

SECTION AVERAGE: 1.3679 .4

$384 \quad 46$

TOP OF SECTION 87.40 METERS SUB-BOTTOM

$\mathrm{RHO}$
1.34

$0.0 \mathrm{CM}: 1.3480 .7$

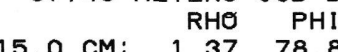

30.0 $\mathrm{RHO} \mathrm{PHI}$

75.0 CM: 1.3381 .2

90.0 CM: 1.3381 .3

$\begin{array}{rrr}105.0 \mathrm{CM}: & 1.36 & 79.5 \\ 105 & 1.32 & 81.8\end{array}$

SECTION AVERAGE:

TOP OF SECTION

89. OO METERS SUB-BOTTOM

RHO PHI RHO PHI RHO PHI

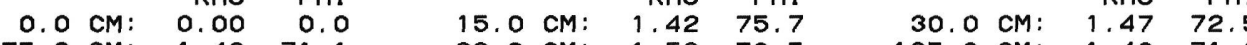

SECTION AVERAGE: 1.4871 .8

38452

TOP OF SECTION 90.50 METERS SUB-BOTTOM

O. $\mathrm{CM}$ :

RHO PHI

5. $\mathrm{CM}: \quad \mathrm{RHO}$ PHI

$30.0 \mathrm{cM}: \quad \begin{array}{r}\mathrm{RHO} \\ 1.50\end{array}$

$\begin{array}{ll}.45 & 74.1 \\ .49 & 71.3\end{array}$

90.0 CM: 1.49 71.1

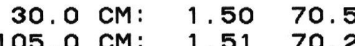

SECTION AVERAGE: $\quad 1.5071 .1$

38453

TOP OF SECTION 92.00 METERS SUB-BOTTOM

$0.0 \mathrm{cM}: \quad \begin{array}{r}\mathrm{RHO} \\ \mathrm{PHI}\end{array}$

75.0 CM: 1.5362 .7

RHO PHI

$\begin{array}{lll}15.0 \mathrm{CM}: & 1.54 & 68.3 \\ 90.0 \mathrm{CM}: & 1.73 & 56.3\end{array}$

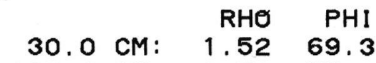

105.0 CM: 1.7157 .8

RHO PHI

SECTION AVERAGE: 1.63 63.0
45. $\mathrm{CM}: 2 \mathrm{RHO} \mathrm{PHI}$ $\begin{array}{rrr}45.0 \text { CM: } & 2.20 & 27.9 \\ 120.0 \text { CM: } & 2.23 & 26.0\end{array}$

(120.0 $\mathrm{cM}: 12.23 .26 .0$

$45.0 \mathrm{~cm}$ RHO PHI

$120.0 \mathrm{cM}: \quad 1.23 \quad 87.3$

$45 . \mathrm{CHO}$ PHI $\begin{array}{rrr}45.0 \mathrm{CM}: & 1.30 & 82.9 \\ 120.0 \mathrm{CM}: & 1.22 & 88.3\end{array}$

45. $\mathrm{RHO}$ PHI $\begin{array}{rrr}\text { 120.0 CM: } & 1.28 & 84.2 \\ & & \end{array}$ $\begin{array}{rll}\text { 45.0 CM: } & 1.31 & 82.4 \\ 120.0 \mathrm{CM}: & 1.34 & 80.8\end{array}$

$0>[2.65 / 6.61 \mathrm{C} 0 / 1.5 / 4]$ 60.0 CM: $2.22 \quad 26.5$ 135. $\mathrm{OCM}: 2.20 \quad 28.0$

$0>[2,65 / 6,61 \mathrm{C} 0 / 1,5 / 4]$ $60.0 \mathrm{CM}: \quad \begin{array}{rr}\mathrm{RHO} & \mathrm{PHI} \\ 1.24 & 87.0\end{array}$ $135.0 \mathrm{CM}: \quad 1.30 \quad 83.2$

$0>[2 . € 5 / 6.61 \mathrm{C} 0 / 1.5 / 4]$ $\begin{array}{rrr}60.0 \mathrm{CM}: & \text { RHO } & \text { PHI } \\ 1.27 & 84.7\end{array}$ $0>[2.65 / 6.61 \mathrm{C} \quad 0 / 1.5 / 4]$ 60.0 CM: $1.27 \quad 84.8$ 135.0 CM: 1.2586 .2

$0>[2.65 / 6.61 C \quad 0 / 1.5 / 4]$

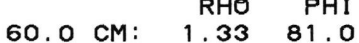
$135.0 \mathrm{CM}: 1.3381 .1$

$0>[2.65 / 6.61 \mathrm{C} 0 / 1.5 / 4]$ 45.0 cM: $\begin{array}{rr}\text { RHO } & \text { PHI } \\ 1.36 & 79.2\end{array}$

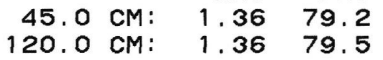
$60.0 \mathrm{cM}: \quad \begin{array}{rr}\mathrm{RHO} & \mathrm{PHI} \\ 1.36 & 79.2\end{array}$ 135. $\mathrm{cm}: 1.37-78.5$

$0>[2.65 / 6.61 \mathrm{C} 0 / 1.5 / 4]$ 45. O cM: $\begin{array}{rr}\text { RHO } & \mathrm{PHI} \\ 1.36 & 79.2\end{array}$ $\begin{array}{rrr}45.0 \mathrm{CM}: & 1.36 & 79.2 \\ 120.0 \mathrm{CM}: & 1.36 & 79.6\end{array}$ $60.0 \mathrm{~cm}: \quad \begin{array}{r}\mathrm{RHO} \\ \mathrm{PHI}\end{array}$ $135.0 \mathrm{CM}: 1.3878 .2$

$0>[2.65 / 6.61 \mathrm{C} 0 / 1.5 / 4]$ 45.0 cM: $\begin{array}{rrr}\text { RHO } & \text { PHI } \\ 1.51 & 70.4\end{array}$ $\begin{array}{lll}120.0 \mathrm{CM}: \quad 1.47 & 72.4\end{array}$

60.0 CM: $1.47 \quad 72.8$

$0>[2.65 / 6.61 \mathrm{C} 0 / 1.5 / 4]$

45.0 CM: $\quad \begin{array}{r}\text { RHO } \\ 1.51\end{array}$

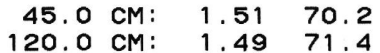
$\begin{array}{rrr}60.0 \mathrm{cM}: & \text { RHO } & \mathrm{PHI} \\ & 1.52 & 69.6\end{array}$ $0>[2.65 / 6,61 \mathrm{C} 0 / 1,5 / 4]$ 45. $\mathrm{cM}: \quad$ RHO 54 120.0 CM: 1.8151 .8 60.0 CM: 1.5965 .3 $135.0 \mathrm{CM}: \quad 1.71 \quad 57.9$ 


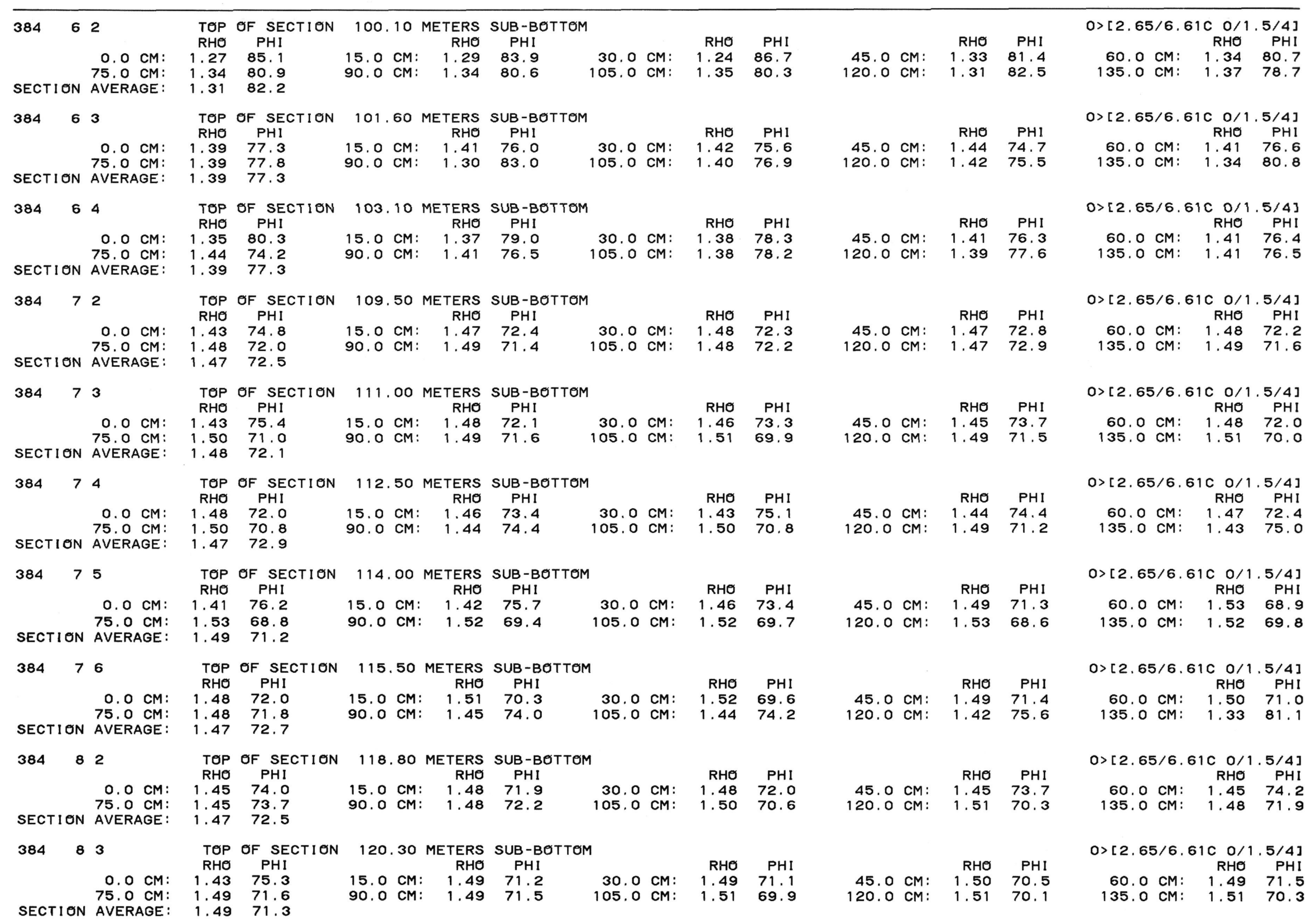


30. $\mathrm{CM}: \quad \mathrm{RHO} \mathrm{PHI}$ $\begin{array}{rrrrrr}\text { 90.0 CM: } & 1.48 & 65.6 & 30.0 \mathrm{CM}: & 1.62 & 63.5 \\ & & & & & \end{array}$

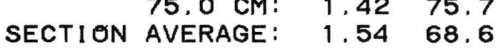

$384 \quad 8 \quad 5$

TOP OF SECTION 123.30 METERS SUB-BOTTOM $\begin{array}{rrrr}0.0 & \text { CM: } & \text { RHO } & \text { PHI } \\ 75.0 & \text { CM: } & 1.46 & 72.4 \\ 73.1\end{array}$ RHO PHI AVERAGE: $1.45 \quad 73.7$

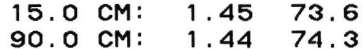
30. $\mathrm{CM}: \begin{array}{rr}\mathrm{RHO} & \mathrm{PHI} \\ 1.45 & 73.6\end{array}$

TOP OF SECTION 127.35 METERS SUB-BOTTOM

$384 \quad 9 \quad 1$

$\begin{array}{rrr}0.0 \mathrm{CM}: & 1.47 & 72.6\end{array}$

$\begin{array}{rrr}15.0 \mathrm{cM}: & \text { RHO } & \text { PHI } \\ 1.46 & 73.2\end{array}$

90.0 CM: 1.4473 .2

30. 0 cM: RHO PHI

$1.48 \quad 71.9$

SECTION AVERAGE:

TOP OF SECTION 128.85 METERS SUB-BOTTOM

$384 \quad 92$

$\begin{array}{rrr}0.0 \mathrm{CM}: & \text { RHO } & \mathrm{PHI} \\ & 1.50 & 70.9\end{array}$

$15.0 \mathrm{cM}: \quad 1.54 \quad 68.1$

30. O cM: RHO PHI

1.6164 .0

90.0 cM: 1.5865 .9

105.0 CM: 1.6164 .2

SECTION AVERAGE

TOP OF SECTION 130.35 METERS SUB-BOTTOM

38493

$\begin{array}{rrr}0.0 \mathrm{CM}: & 1.56 & 66.8 \\ 75.0 \mathrm{CM}: & 1.47 & 72.6\end{array}$ $15.0 \mathrm{CM}: \quad \begin{array}{r}\mathrm{RHO} \\ \mathrm{PHI}\end{array}$

$30.0 \mathrm{~cm}: \quad \begin{array}{r}\mathrm{RHO} \\ \mathrm{PHI}\end{array}$ $90.0 \mathrm{CM}: 1.4574 .0$

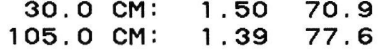

SECTION AVERAGE: 1.4971 .7

38495

TOP OF SECTION 133.35 METERS SUB-BOTTOM

RHO PHI

$\begin{array}{rll}0.0 \mathrm{CM}: & 1.45 & 74.1\end{array}$

15.0 RHO PHI

30. 0 CM: $\quad \begin{array}{rr}\text { RHO } & \mathrm{PHI}\end{array}$ 90.0 CM: $1.49 \quad 71.5$

105.0 CM: $1.48 \quad 71.7$

SECTION AVERAGE: $1.48 \quad 71.8$

38496

TOP OF SECTION 134.85 METERS SUB-BOTTOM

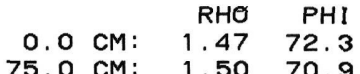

SECTION AVERAGE: 1.4971 .3

$\begin{array}{rrr}15.0 \mathrm{cM}: & \text { RHO } & \text { PHI } \\ 1.49 & 71.3\end{array}$

$30 . \mathrm{CM}: \mathrm{RHO} \mathrm{PHI}$

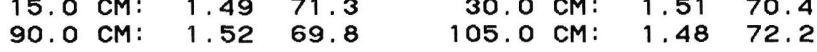

TOP OF SECTION 137.90 METERS SUB-BOTTOM

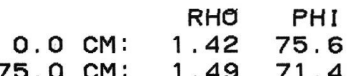

$\begin{array}{rrr}15.0 \mathrm{cM}: & \mathrm{RHO} & \mathrm{PHI} \\ & 1.46 & 73.5\end{array}$

30.0 CM: $\begin{array}{rr}\text { RHO } & \mathrm{PHI} \\ 1.31 & 82.4\end{array}$ 90.0 CM: $1.48 \quad 71.8$

$\begin{array}{lll}105.0 \mathrm{CM}: & 1.51 & 70.1\end{array}$

SECTION AVERAGE: 1.4474 .2

TOP OF SECTION 139.40 METERS SUB-BOTTOM

$384 \quad 103$

RHO PHI

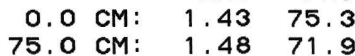

$\begin{array}{rrr}15.0 \mathrm{CM}: & \mathrm{RHO} & \mathrm{PHI} \\ & 1.46 & 73.5\end{array}$

30. $\mathrm{CM}: \begin{array}{rr}\mathrm{RHO} & \mathrm{PHI} \\ 1.46 & 73.0\end{array}$

90.0 CM: 1.4374 .9

$105.0 \mathrm{CM}: \quad 1.46 \quad 73.0$

SECTION AVERAGE: 1.4673 .3

$384 \quad 104$

TOP OF SECTION 140.90 METERS SUB-BOTTOM

$\begin{array}{rrr}0.0 \mathrm{CM}: & \mathrm{RHO} & \mathrm{PHI} \\ 75.0 \mathrm{CM}: & 1.45 & 74.3 \\ 73.9\end{array}$

150.90 PHI

30.0 CM: $\begin{array}{rrr}\text { RHO } & \text { PHI } \\ 1.46 & 73.1\end{array}$

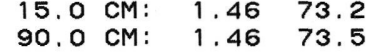

$\begin{array}{rrr}30.0 \mathrm{CM}: & 1.46 & 73.1 \\ 105.0 \mathrm{CM}: & 1.45 & 73.9\end{array}$
45.0 CM: $\quad$ RHO PHI $\begin{array}{rrr}120.0 \mathrm{CM}: & 1.57 & 66.2 \\ & 1.55 & 67.7\end{array}$

$45.0 \mathrm{CM}: \quad \mathrm{RHO} \quad \mathrm{PHI}$ $\begin{array}{rrr}\text { 120.0 CM: } & 1.42 & 72.6 \\ & & \end{array}$

45. $0 \mathrm{CM}: \quad$ RHO PHI 120.0 CM: $\quad 1.46 \quad 73.1$

45.0 CM: 1.62 RHO

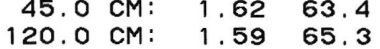

45. O CM: $\quad \begin{array}{r}\text { RHO PHI } \\ \text { PH }\end{array}$ 120.0 CM: 1.4574 .1

45.0 CM: $\quad \begin{array}{rr}\text { RHO } & \mathrm{PHI}\end{array}$ 120.0 CM: $1.48 \quad 71.9$

45. $\mathrm{CM}:$ RHO $\mathrm{PHI}$

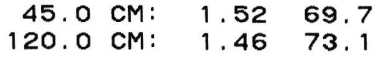

45. $\mathrm{CM}: \begin{array}{rr}\mathrm{RHO} & \mathrm{PHI} \\ 1.40 & 77.0\end{array}$ 120.0 CM: $1.46 \quad 73.1$

45.0 CM: $\begin{array}{rr}\text { RHO } & \text { PHI } \\ 1.45 & 74.0\end{array}$ 120.0 CM: 1.4871 .8

45. O CM: $\quad$ RHO PHI 120.0 CM: 1.4573 .6
$0>[2.65 / 6.61 \mathrm{C} 0 / 1.5 / 4]$ $60.0 \mathrm{~cm}: \quad \begin{array}{rr}\mathrm{RHO} & \mathrm{PHI} \\ 1.58 & 65.5\end{array}$ $135.0 \mathrm{CM}: 1.5170 .0$

$0>[2.65 / 6.61 \mathrm{C} \quad 0 / 1.5 / 4]$ $60.0 \mathrm{~cm}: \quad \begin{array}{rr}\mathrm{RHO} & \mathrm{PHI} \\ 1.43 & 75.2\end{array}$ 135.0 CM: $1.46 \quad 73.1$

$0>[2.65 / 6.61 \mathrm{C} 0 / 1.5 / 4]$ $60.0 \mathrm{cM}: \quad \begin{array}{rr}\mathrm{RHO} & \mathrm{PHI} \\ 61.49 & 71.6\end{array}$ $0>[2.65 / 6.61 \mathrm{C} \quad 0 / 1.5 / 4]$ $60.0 \mathrm{~cm}$ RHO PHI $135.0 \mathrm{CM}: 1.5567 .5$

$0>[2.65 / 6.61 \mathrm{C} 0 / 1.5 / 4]$ $60.0 \mathrm{~cm}: \quad \begin{array}{rr}\mathrm{RHO} & \mathrm{PHI}\end{array}$ $135.0 \mathrm{CM}: 1.47 \quad 72.4$

$0>[2.65 / 6.61 \mathrm{C} \quad 0 / 1.5 / 4]$ 60.0 CM: 1.4971 .4 135.0 CM: 1.4971 .7

$0>[2.65 / 6.61 \mathrm{C} 0 / 1.5 / 4]$

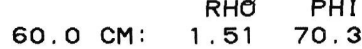
$135.0 \mathrm{CM}: 1.45$ 73.?

$0>[2.65 / 6.61 \mathrm{C} 0 / 1.5 / 4]$ $60.0 \mathrm{~cm}: \quad \begin{array}{rr}\mathrm{RHO} & \mathrm{PHI} \\ 1.43 & 74.9\end{array}$ 135.0 CM: 1.4673 .1

$0>[2.65 / 6.61 \mathrm{C} \quad 0 / 1.5 / 4]$ 60.0 CM: $1.49 \quad 71.3$ $0>[2.65 / 6.61 \mathrm{C} 0 / 1.5 / 4]$ $\begin{array}{rrr}60.0 \mathrm{cM}: & \mathrm{RHO} & \mathrm{PHI} \\ 1.42 & 75.8\end{array}$ $135.0 \mathrm{CM}: 1.4374 .8$ 


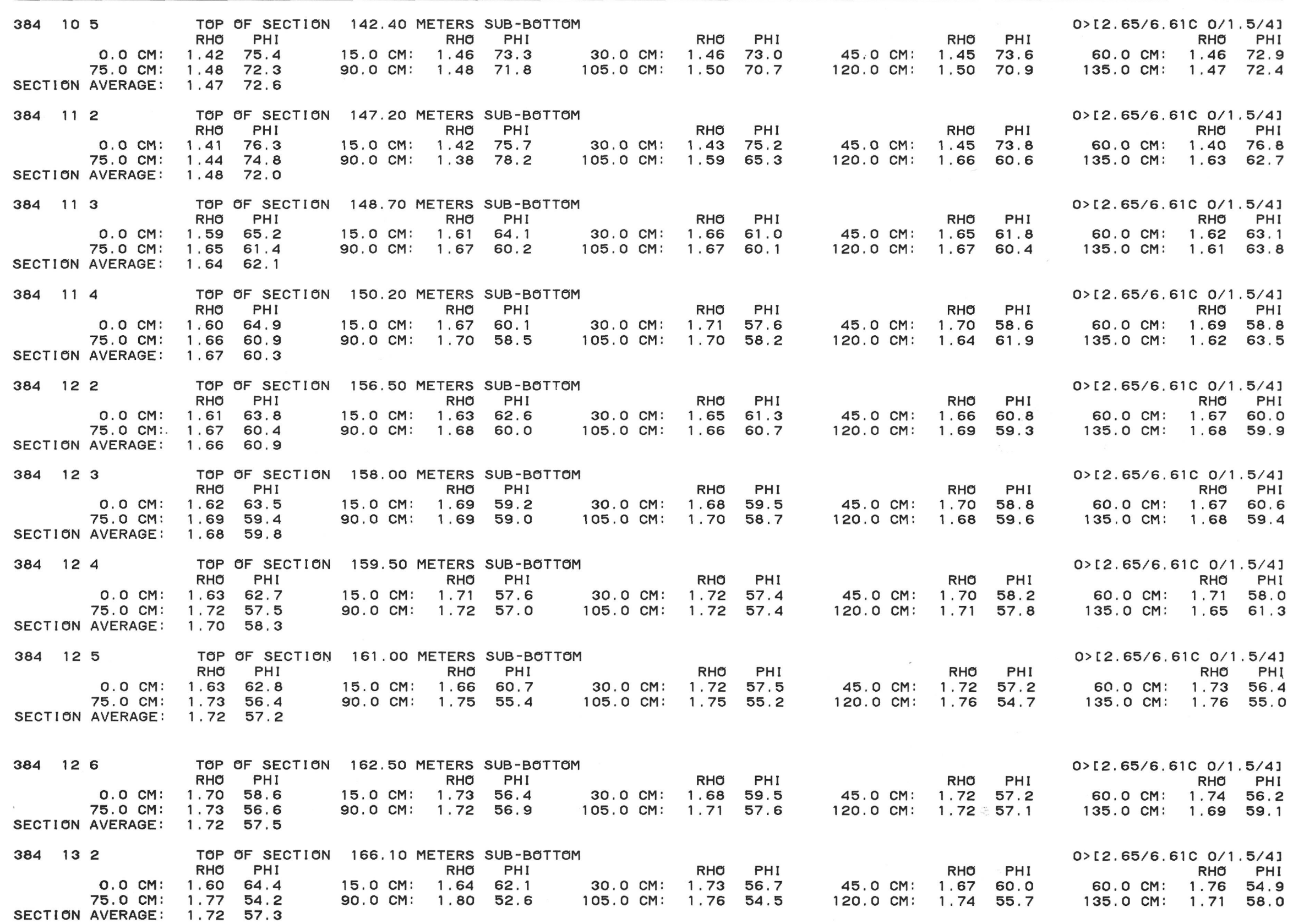


TOP OF SECTION 167.60 METERS SUB-BOTTOM

RHO PHI

O. 0 CM:

$75.0 \mathrm{CM}$

SECTION AVERAGE

$384 \quad 134$

$0.0 \mathrm{CM}:$
$75.0 \mathrm{CM}:$

SECTION AVERAGE:

$384 \quad 135$

\section{O. $0 \mathrm{CM}$ :}

SECTION AVERAGE:

$384 \quad 136$

O. $0 \mathrm{CM}$

75. OM

$384 \quad 142$

TOP OF SECTION 175.10 METERS SUB-BOTTOM
RHO PHI

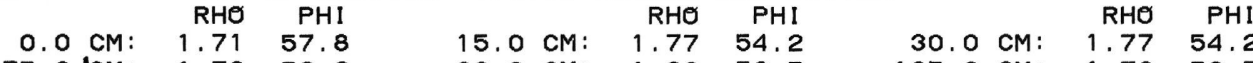

SECTION AVERAGE: $1.79 \quad 52.6$

$384 \quad 143$

TOP OF SECTION 176.60 METERS SUB-BOTTOM

$0.0 \mathrm{CM}: \quad \begin{array}{rr}\text { RHO } & \text { PHI } \\ 0.78 & 53.3\end{array}$

$75.0 \mathrm{CM}: 1.80 \quad 52.5$

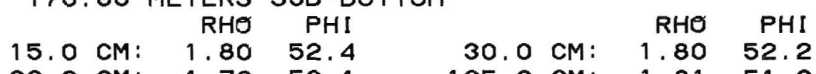

SECTION AVERAGE

$384 \quad 15 \quad 1$

TOP OF SECTION 183.25 METERS SUB-BOTTOM

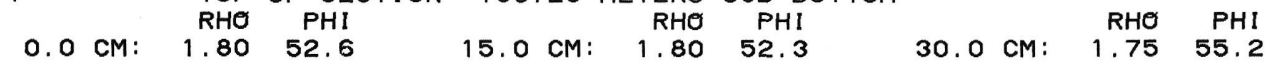

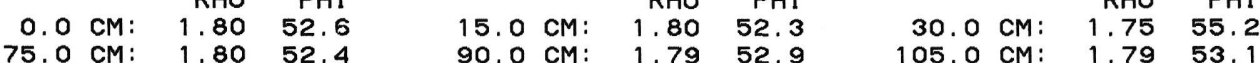

$\begin{array}{lll}75.0 \mathrm{CM}: & 1.80 & 52.4 \\ \text { SECTION AVERAGE: } & 1.78 & 53.7\end{array}$

384152

TOP OF SECTION 184.75 METERS SUB-BOTTOM

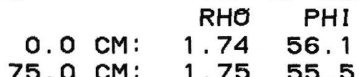

15.0 CM: RHO PHI

$\begin{array}{lll}15.0 \mathrm{CM}: & 1.79 & 53.0 \\ 90.0 \mathrm{CM}: & 1.77 & 54.2\end{array}$

30.0 CM: $\begin{array}{rrr}\text { RHO } & \text { PHI } \\ 1.77 & 54.1\end{array}$

105.0 CM: $1.81 \quad 51.9$

SECTION AVERAGE: $1: 7754.2$

$384 \quad 153$

TOP OF SECTION 186.25 METERS SUB-BOTTOM

$0.0 \mathrm{CM}$ :

RHO $\mathrm{PHI}$

15.0 CM: $\quad \begin{array}{rrr}\text { RHO } & \text { PHI } \\ 1.83 & 50.7\end{array}$

$\begin{array}{rrr}\text { 30. } & \text { RHO } & \text { PHI } \\ 105.0 & 1.85 & 49.2 \\ 109 & 1.78 & 53.4\end{array}$

1.7754 .2

90.0 CM: $1.76 \quad 54.6$

SECTIOON AVERAGE:

TOP OF SECTION 187.75 METERS SUB-BOTTOM

$384 \quad 154$

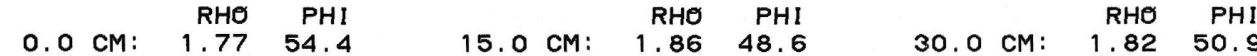

$\begin{array}{rrrrrrrrr}0.0 \mathrm{CM}: & 1.77 & 54.4 & 15.0 \mathrm{CM}: & 1.86 & 48.6 & 30.0 \mathrm{CM}: & 1.82 & 50.9 \\ 75.0 \mathrm{CM}: & 1.85 & 49.1 & 90.0 \mathrm{CM}: & 1.84 & 50.0 & 105.0 \mathrm{CM}: & 1.84 & 50.1\end{array}$

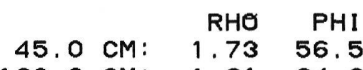
$120.0 \mathrm{CM}: 1.6164 .2$

45. $\mathrm{CM}:$ RHO PHI

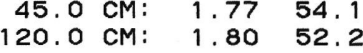

45. CM: RHO PHI $\begin{array}{rll}\text { 45.0 CM: } & 1.80 & 52.0 \\ 120.0 \mathrm{CM}: & 1.76 & 54.8\end{array}$

45. $\mathrm{RHO} \mathrm{PHI}$ 120.0 CM: 1.68 59.4

45.0 cM: 1.76 PHI 120.0 CM: 1.78 53.9

45.0 CM: $\quad \begin{array}{rr}\text { RHO } & \text { PHI } \\ 1.81 & 51.5\end{array}$ $120.0 \mathrm{CM}: 1.8052$.

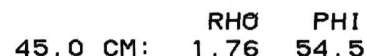
$\begin{array}{rrr}45.0 \mathrm{CM}: & 1.76 & 54.5 \\ 120.0 \mathrm{CM}: & 1.76 & 54.8\end{array}$

45. $\mathrm{RHO} \mathrm{PHI}$ $\begin{array}{rrr}45.0 \mathrm{CM}: & 1.78 & \mathbf{5 3} .5 \\ 120.0 \mathrm{CM}: & 1.75 & \mathbf{5 5 . 2}\end{array}$

RHO PHI 45. O CM: 1.85 49.4

45. O CM: RHO PHI 120.0 CM: 1.8251.
$0>[2,65 / 6,61 \mathrm{C} 0 / 1,5 / 4]$ $60.0 \mathrm{cM}: \quad \mathrm{RHO}$ PHI $135.0 \mathrm{CM}: 1.67$ 60.2

$0>[2.65 / 6.61 \mathrm{C} 0 / 1.5 / 4$ $60.0 \mathrm{cM}: \quad \begin{array}{r}\mathrm{RHO} \\ \mathrm{PHI}\end{array}$ $135.0 \mathrm{cM}: 1.80 \mathrm{52.4}$

0) $[2.65 / 6.61 \mathrm{C} \quad 0 / 1.5 / 4]$ $60.0 \mathrm{cM}: 1.82 \quad 51.0$ $135.0 \mathrm{CM}: \quad 1.75 \quad 55.3$

$0>[2.65 / 6.61 \mathrm{C} 0 / 1.5 / 4]$ $60.0 \mathrm{cM}: \quad \mathrm{RHO} \mathrm{PH}$ $135.0 \mathrm{CM}: 1.5965 .2$

$0>[2.65 / 6.61 \mathrm{C} \quad 0 / 1.5 / 4]$ $60.0 \mathrm{~cm}: \quad \begin{array}{r}\mathrm{RHO} \\ \mathrm{PHI}\end{array}$ $135.0 \mathrm{cM}: 1.79 \quad 52.9$

$0>[2.65 / 6.61 \mathrm{C} \quad 0 / 1.5 / 4]$ 60.0 cM: $1.81 \quad 52.0$ $135.0 \mathrm{CM}: 1.80 \quad 52.5$

$0>[2.65 / 6.61 \mathrm{C} \quad 0 / 1.5 / 4]$ $60.0 \mathrm{cM}: \quad \begin{array}{rr}\mathrm{RHO} & \mathrm{PH} I \\ 1.77 & 53.9\end{array}$ $135.0 \mathrm{CM}: 1.7655 .0$

$0>[2.65 / 6.61 \mathrm{C} \quad 0 / 1.5 / 4]$ $60.0 \mathrm{cM}: \quad \begin{array}{rr}\text { RHO } & \mathrm{PH} \\ 1.79 & 52.8\end{array}$ 135. O CM: 1.7455.

$0>[2.65 / 6.61 \mathrm{C} \quad 0 / 1.5 / 4]$ 60.0 CM: 1.77 53.9 $0>[2.65 / 6.61 \mathrm{C} \quad 0 / 1.5 / 4]$ RHO PHI $135.0 \mathrm{CM}: 0.00 \quad 0.0$ 


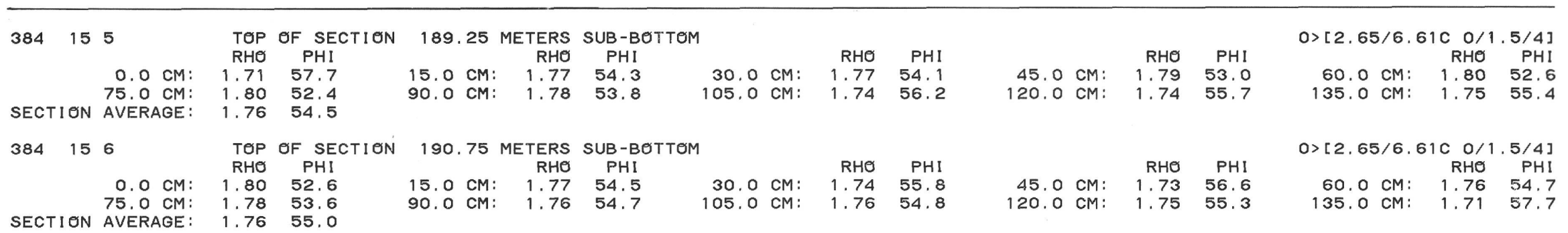

384221

TOP OF SECTION 324.80 METERS SUB-BøTTOM

$0=[2.65 / 6.61 \mathrm{C} 0 / 1.5 / 4] \quad 115=[2.65 / 5.80 \mathrm{C} \quad 0 / 1.5 / 4] \quad 120=[2.65 / 5.90 \mathrm{C} 0 / 1.5 / 4] \quad 130=[2.65 / 5.93 \mathrm{C} 0 / 1.5 / 4]$

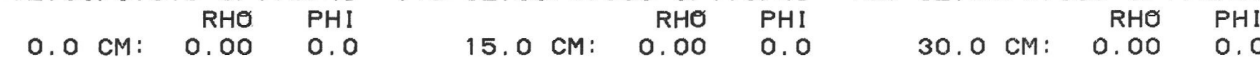

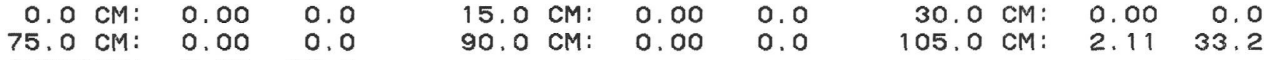
$\begin{array}{rr}75.0 \text { CM: } & 0.00 \\ \text { SECTI ON AVERAGE: } & 2.20 \quad 27.5\end{array}$

RHO PHI

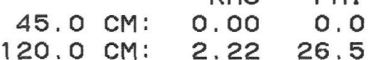
384222

TOP OF SECTION 326.30 METERS SUB-BOTTOM

$0=[2.65 / 6.61 \mathrm{C} 0 / 1.5 / 4] \quad 12=[2.65 / 5.90 \mathrm{C} \quad 0 / 1.5 / 4] \quad 25=[2.65 / 5.85 \mathrm{C} 0 / 1.5 / 4]$ $50=[2,65 / 2$ $90=[2.65 / 6.08 \mathrm{C} 0 / 1.5 / 4]$ $55=[2,65 / 5,950 / 1,5 / 4] \quad 65=[2,65 / 5,850 / 0 / 1,5 / 4]$ $135=[2.65 / 5.82 C 0 / 1.5 / 4]$ $5=[2,65 / 6,00 \mathrm{C} 0 / 1,5 / 4] \quad 105=[2,65 / 5,88 \mathrm{C} 0 / 1,5 / 4]$

$35=[2.65 / 5.900 \quad 0 / 1.5 / 4]$ $75=[2,65 / 5,05 \mathrm{C} \quad 0 / 1,5 / 4]$ $120=[2,65 / 6,61 \mathrm{C} 0 / 1,5 / 4]$

$$
\begin{array}{rrr}
0.0 \mathrm{CM}: & \mathrm{RHO} & \mathrm{PHI} \\
75.0 \mathrm{CM}: & 2.23 & 26.1 \\
& 2.13 & 32.1
\end{array}
$$

15. $\mathrm{CM}: \mathrm{RHO}$ PHI

$\begin{array}{llll}15.0 & \mathrm{CM}: & 2.17 & 29.2 \\ 90.0 \mathrm{CM}: & 2.19 & 28.3\end{array}$

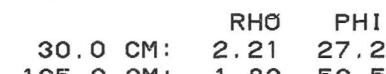

SECTION AVERAGE: $2.05 \quad 37.2$

TOP OF SECTION 25.30 METERS SUB-BOTTOM

$385 \quad 13$

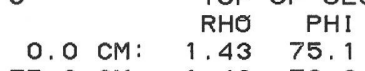

$\begin{array}{rrr}\text { O.0 CM: } & 1.43 & 75.1 \\ \text { 75.O CM: } & 1.46 & 73.3 \\ \text { AVERAGE: } & 1.47 & 72.7\end{array}$

15.0 CM: $\quad 1.44 \quad 74.5$

30.0 $\mathrm{RHO}$ PHI

90.0 CM: $1.49 \quad 71.3$

105. O CM: $\quad 1.4474 .3$

SECTION AVE

TOP OF SECTION 26.80 METERS SUB-BOTTOM

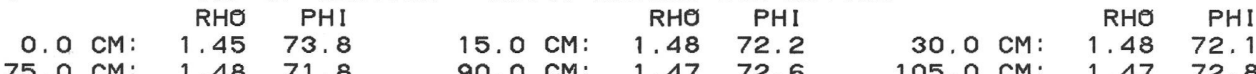

$\begin{array}{lll}75.0 \mathrm{CM}: & 1.48 & 71.8 \\ \text { SECTION AVERAGE: } & 1.47 & 72.5\end{array}$

90.0 CM: $1.47 \quad 72.6$

TOP OF SECTION 68.35 METERS SUB-BOTTOM

38526

$0.0 \mathrm{CM}: 1.27 \quad 84.9$

75.0 CM: 1.29 83.4

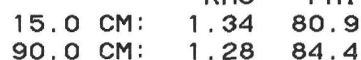

30. O CM: $\quad \begin{array}{rrr}\text { RHO } & \text { PHI } \\ 1.32 & 81.8\end{array}$

$105.0 \mathrm{CM}: \quad 1.28$ 84.1

SECTION AVERAGE: $1.30 \quad 83.3$

TOP OF SECTION 138.00 METERS SUB-BOTTOM

$\begin{array}{rrr}0.0 \mathrm{CM}: & \text { RHO } & \text { PHI } \\ 75.0 & 88.0\end{array}$

$\begin{array}{lll}75.0 \mathrm{CM}: & 1.18 & 90.5 \\ \text { SECTION AVERAGE: } & 1.18 & 90.7\end{array}$

15.0 CM: $1.20 \quad 89.0$

30.0 CM: $\begin{array}{rrr}\mathrm{RHO} & \mathrm{PHI} \\ 1.20 & 89.4\end{array}$

90.0 CM: 1.14 93.1 $105.0 \mathrm{CM}: 1.15 \quad 92.3$

38543

TOP OF SECTION 139.50 METERS SUB-BOTTOM

$\begin{array}{rrr}0.0 \mathrm{CM}: & \text { RHO } & \mathrm{PHI} \\ 7.26 & 85.5\end{array}$

SECTION AVERAGE: 1.2586 .2 $\begin{array}{rrr}15.0 \mathrm{CM}: & \text { RHO } & \text { PHI } \\ 1.22 & 88.1\end{array}$

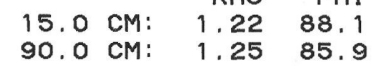
$\begin{array}{rrr}30.0 \mathrm{CM}: & 1.24 & 86.5 \\ 105.0 \mathrm{CM}: & 1.23 & 87.1\end{array}$

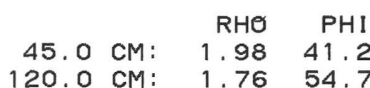

$\begin{array}{rll}45.0 \mathrm{CM}: & 1.46 & 73.3 \\ 120.0 \mathrm{CM}: & 1.52 & 69.4\end{array}$

45.0 $\mathrm{CM}: \quad \mathrm{RHO} \mathrm{PHI}$

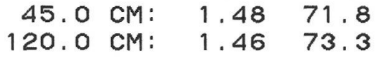

$\begin{array}{lll}120.0 \mathrm{CM}: & 1.29 & 83.4 \\ & & \end{array}$

45.0 CM: $\begin{array}{rr}\text { RHO } & \text { PHI } \\ 1.21 & 88.9\end{array}$ $\begin{array}{lll}120.0 \text { CM: } & 1.15 & 92.2\end{array}$

45. O CM: RHO PHI $\begin{array}{rrr}45.0 \mathrm{CM}: & 1.25 & 86.1 \\ 120.0 \mathrm{CM}: & 1.24 & 87.0\end{array}$
45. $\mathrm{CM}: \quad \mathrm{RHO} \mathrm{PHI}$

45. O cM: $\quad$ RHO PHI
$140=[2.65 / 5.93 \mathrm{C} 0 / 1.5 / 4]$ $60.0 \mathrm{~cm}: \mathrm{RHO} \quad \mathrm{PH} 1$ 135.0 CM: $2.26 \quad 23.8$

$45=[2.65 / 5.95 \mathrm{C} \quad 0 / 1.5 / 4]$ $80=[2,65 / 5,91 \mathrm{C} 0 / 1,5 / 4]$ . $0 / 1,5 / 4]$ $\begin{array}{rrr}60.0 \mathrm{CM}: & \text { RHO } & \mathrm{PHI} \\ & 1.83 & 50.2\end{array}$ 135.0 CM: 2.0536 .7

$0>[2.65 / 6,61 \mathrm{C} 0 / 1,5 / 4]$

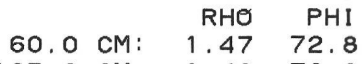

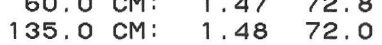

$0>[2.65 / 6.61 \mathrm{C} \quad 0 / 1.5 / 4]$ $\begin{array}{rrr}60.0 \mathrm{CM}: & \mathrm{RHO} & \mathrm{PHI} \\ 135.08 & 72.0\end{array}$ $0>[2.65 / 6.61 \mathrm{C} 0 / 1.5 / 4]$ 60.0 cM: $\begin{array}{rr}\text { RHO } & \text { PHI } \\ 1.26 & 85.5\end{array}$ $135.0 \mathrm{CM}: 1.37 \quad 79.0$

$0>[2.65 / 6.61 \mathrm{C} 0 / 1.5 / 4]$ $60.0 \mathrm{~cm}: \quad \begin{array}{rr}\mathrm{RHO} & \mathrm{PHI} \\ 1.16 & 91.9\end{array}$ 135.0 CM: 1.1691 .6

$0>[2.65 / 6.61 \mathrm{C} \quad 0 / 1.5 / 4]$ $60.0 \mathrm{cM}: \quad \begin{array}{rr}\mathrm{RHO} & \mathrm{PHI} \\ 1.26 & 85.4\end{array}$ 135.0 CM: $1.27 \quad 85.0$ 
45.0 CM: $\quad \begin{array}{rr}\text { RHO } & \text { PHI } \\ 1.23 & 87.7\end{array}$ 20.0 CM: $1.26 \quad 85.6$

$385 \quad 53$

TOP OF SECTION 149.10 METERS SUB-BOTTOM O. $0 \mathrm{CM}:$ 149.10 METERS SUB-BOTTOM RHO PHI PHI $1.30 \quad 82.9$ $15.0 \mathrm{cM}: \quad 1.32 \quad 81.9$

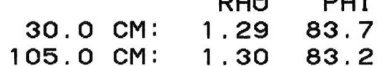
SECTION AVERAGE: 1.29

$\begin{array}{ll}\text { TOP OF SECTION } & 150.60 \text { METERS SUB-BOTTOM } \\ \text { RHO PHI } & \text { RHO PHI }\end{array}$

$385 \quad 54$

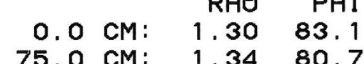

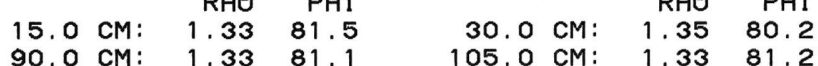

30.0 $\mathrm{CM}: \mathrm{RHO} \mathrm{PHI}$

SECTION AVERAGE: $1.33 \quad 81.4$

$385 \quad 8 \quad 1$

TOP OF SECTION 174.60 METERS SUB-BOTTOM

$0.0 \mathrm{cM}: \mathrm{RHO} \mathrm{PHI}$

75.0 CM: 0.00 O.0

SECTION AVERAGE: 1.5170 .2

15. O CM: 0.00 PHI

$30.0 \mathrm{~cm}: \quad \mathrm{RHO} \quad \mathrm{PHI}$

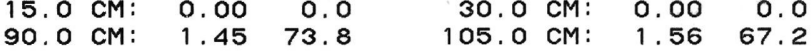

$385 \quad 85$

TOP OF SECTION 180.60 METERS SUB-BOTTOM

RHO PHI

$0.0 \mathrm{CM}: 0.00 \quad 0.0$ $\begin{array}{lll}75.0 \mathrm{CM}: & 1.54 & 68.2 \\ \text { SECTION AVERAGE: } & 1.53 & 69.0\end{array}$

$15.0 \mathrm{~cm}: \mathrm{RHO}$ PHI

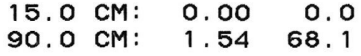

30. $0 \mathrm{~cm}: \quad \mathrm{RHO} \quad \mathrm{PHI}$

TOP OF SECTION 184.10 METERS SUB-BOTTOM

3859

RHO PHI $\begin{array}{rrr}0.0 \mathrm{CM}: & 0.00 & 0.0 \\ 75.0 \mathrm{CM}: & 1.29 & 83.9\end{array}$ SECTION AVERAGE: 1.3878 .4

\section{0 cM: $\quad 0.00 \quad$ PHI} $90.0 \mathrm{CM}: \quad 1.3878 .2$

30.0 $\mathrm{RHO} \quad \mathrm{PHI}$ $\begin{array}{rrr}30.0 \mathrm{CM}: & 0.00 & 0.0 \\ 105.0 \mathrm{CM}: & 1.37 & 78.5\end{array}$

TOP OF SECTION 204.50 METERS SUB-BOTTOM

$385 \quad 112$

RHQ $\begin{array}{rrr}0.0 \mathrm{CM}: & 1.50 & 70.7 \\ 75.0 \mathrm{CM}: & 1.57 & 66.5\end{array}$

SECTION AVERAGE: 1.5269 .3

$385 \quad 122$

TOP OF SECTION 214.00 METERS SUB-BOTTOM

RHO PHI $0.0 \mathrm{CM}: \quad 1.74 \quad 55.9$ $75.0 \mathrm{CM}: \quad 1.77$ 54.1 SECTION AVERAGE: $1.77 \quad 54.2$

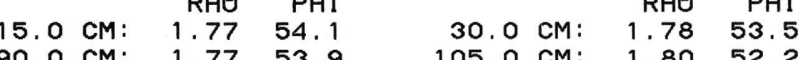

TOP OF SECTION 233.10 METERS SUB-BOTTOM
RHO PHI

$385 \quad 13$

$0.0 \mathrm{CM}: \quad 1.5965 .4$

$75.0 \mathrm{CM}: \quad 1.5465 .1$ $15.0 \mathrm{cM}: \quad 1.72 \quad 57.3$

RHO $\mathrm{PHI}$ 1.5468 .1 90.0 CM: 1.5865 .7 SECTI ON AVERAGE:

TOP OF SECTION 234.60 METERS SUB-BOTTOM

$385 \quad 13 \quad 3$

$0.0 \mathrm{CM}: \quad 1.54 \quad 68.1$

$75.0 \mathrm{CM}: \quad 1.7654 .8$

SECTI ON AVERAGE:
$15.0 \mathrm{CM}: \quad 1.60 \quad 64.6$ $90.0 \mathrm{CM}: 1.76 \quad 54.9$

30.0 $\mathrm{CM}: \quad \mathrm{RHO} \mathrm{PHI}$

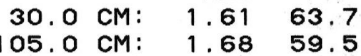

45.0 CM: $\quad \begin{array}{r}\text { RHO } \\ \text { PHI }\end{array}$ 45. $120.1 .28 \quad 84.6$

45.0 cM: $\quad \begin{array}{r}\text { RHO } \\ \text { PHI }\end{array}$ 120.0 CM: 1.3182 .5 $120.0 \mathrm{CM}: 1.5368 .7$

45. O CM: $\begin{array}{rr}\text { RHO } & \text { PHI } \\ 0.00 & 0.0\end{array}$

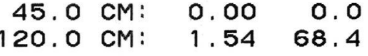

$45.0 \mathrm{~cm}: \mathrm{RHO} \quad \mathrm{PHI}$ (1)

45.0 CM: $\begin{array}{rr}\text { RHO } & \text { PHI } \\ 1.53 & 68.6\end{array}$ 120.0 CM: 1.56 67.6

45. $\mathrm{CM}: 1.76$ PHI 120.0 CM: 1.8154 .6

45. O CM: $\quad \begin{array}{rr}\text { RHO } & \text { PHI }\end{array}$ $120.0 \mathrm{cM}: 0.00 \mathrm{O}$

45.0 CM: $\quad$\begin{tabular}{rrr} 
RHO & PHI \\
\hline & 59.69 &
\end{tabular} $120.0 \mathrm{CM}: 1.6362 .9$

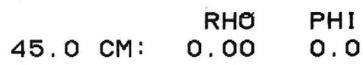

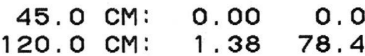

$0>[2.65 / 6.61 \mathrm{C} 0 / 1.5 / 4]$ $60.0 \mathrm{CM}: \quad \begin{array}{r}\mathrm{RHO} \\ 1.22\end{array}$

$0>[2.65 / 6.61 \mathrm{C} 0 / 1.5 / 4]$ $60.0 \mathrm{cM}: \mathrm{RHO} \mathrm{PHI}$ $135.0 \mathrm{cM}: 1.28 \mathrm{8} 4.6$

$0>[2.65 / 6.61 \mathrm{C} 0 / 1.5 / 4]$ $60.0 \mathrm{~cm}: \quad \begin{array}{r}\mathrm{RHO} \\ \mathrm{PHI}\end{array}$ 135.0 CM: 1.3281 .9

$0>[2.65 / 6.61 \mathrm{C} 0 / 1.5 / 4]$ $60.0 \mathrm{CM}: \quad 0.00 \quad 0.0$ $135.0 \mathrm{CM}: \quad 1.43 \quad 75.4$

$0>[2.65 / 6.61 \mathrm{C} \quad 0 / 1.5 / 4]$ $60.0 \mathrm{~cm}: \quad \begin{array}{rr}\mathrm{RHO} & \mathrm{PHI} \\ 1.30 & 83.3\end{array}$ 135.0 CM: 1.50 70.8

$0>[2.65 / 6.61 \mathrm{C} 0 / 1.5 / 4]$ 60.0 cM: $0.00 \quad 0.0$ 135.0 CM: 1.4275 .9

$0>[2.65 / 6.61 \mathrm{C} \quad 0 / 1.5 / 4]$ 60. $\mathrm{CM}: \quad 1.6064 .5$ 135.0 CM: 1.4474 .7

$0>[2,65 / 6.61 \mathrm{C} 0 / 1.5 / 4]$ $60.0 \mathrm{cM}: \quad \mathrm{RHO} \quad \mathrm{PHI}$ 135.0 CM: 1.69 59.3

$0>[2.65 / 6.61 \mathrm{C} 0 / 1.5 / 4]$ $60.0 \mathrm{~cm}: \quad \begin{array}{r}\mathrm{RHO} \\ \mathrm{PHI}\end{array}$ $135.0 \mathrm{~cm}: \quad 0.00 \quad 0.0$

$0>[2.65 / 6.61 \mathrm{C} 0 / 1.5 / 4]$ $60.0 \mathrm{cM}: \quad \begin{array}{rr}\mathrm{RHO} & \mathrm{PHI} \\ 67.72 & 57.5\end{array}$ $135.0 \mathrm{CM}: 1.37 \quad 79.0$ 


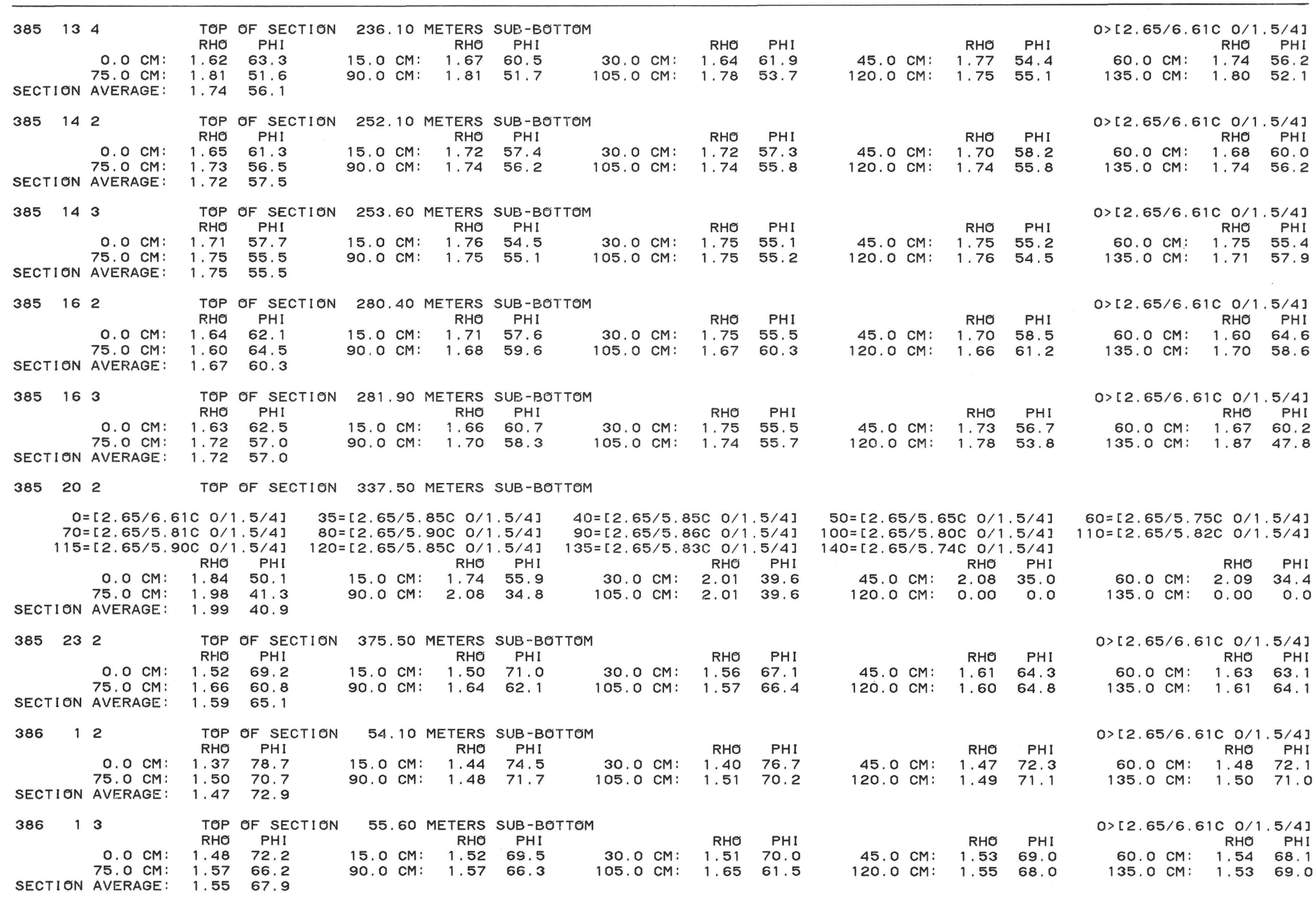


$386 \quad 14$ TOP OF SECTION 57.10 METERS SUB-BOTTOM O. CM: RHO PHI $15.0 \mathrm{cM}$ RHO PHI RHO PHI

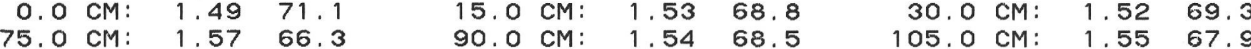
SECTION AVERAGE: 1.5468 .6

TOP OF SECTION 58.60 METERS SUB-BOTTOM

$386 \quad 15$

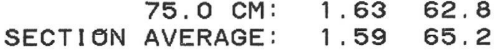

$386 \quad 16$

TOP OF SECTION 60.10 METERS SUB-BOTTOM

RHO PHI RHO PHI

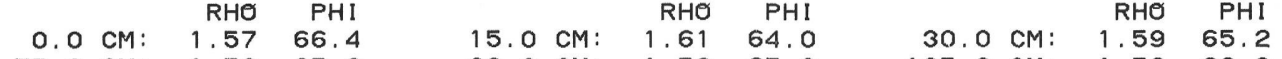

$\begin{array}{lllll}65.0 & 90.0 \mathrm{CM}: 1.5965 .0 \quad 105.0 \mathrm{CM}: 1.56 & 66.9 & & \end{array}$

SECTION AVERAGE: 1.5965 .5

$386 \quad 42$

TOP OF SECTION 148.70 METERS SUB-BOTTOM

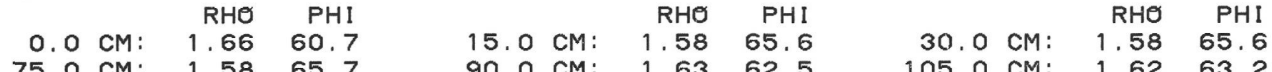

SECTION AVERAGE: 1.6164 .0

TYP OF SECTION 150.20 METERS SUB-BOTTOM

$386 \quad 43$

$\begin{array}{rrr}0.0 \mathrm{CM}: & \text { RHO } & \mathrm{PHI} \\ 75.0 \mathrm{CM}: & 1.61 & 65.0 \\ 75.3 & 64.3\end{array}$

15.0 CM: 1.60 PHO 64.6

30. $\mathrm{CM}: \mathrm{RHO} \mathrm{PHI}$

90.0 CM: $1.65 \quad 64.6$

105. O CM: 1.63 64.9

SECTION AVERAGE

TOP OF SECTION 151.70 METERS SUB-BOTTOM

$386 \quad 4 \quad 4$

O.0 CM: $\quad 1.60 \quad 64.8$

SECTION AVERAGE: 1.66 61.2

30.0 cM: 1.62 RH $90.0 \mathrm{~cm}: 1.74 \quad 56.0 \quad 105.0 \mathrm{~cm}: 1.7455 .8$

38645

TOP OF SECTION 153.20 METERS SUB-BOTTOM

RHO PHI

$\begin{array}{lll}0.0 \mathrm{CM}: & 1.63 & 62.8 \\ 75.0 \mathrm{CM}: & 1.71 & 57.5\end{array}$

$\begin{array}{lll}15.0 \mathrm{CM}: \quad \text { RHO } & \text { PHI } \\ 1.76 & 54.5\end{array}$

SECTION AVERAGE: 1.7058 .3

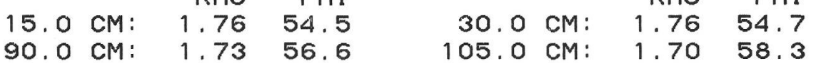

$386 \quad 46$

TOP OF SECTION 154.70 METERS SUB-BOTTOM

RHO PHI

$0.0 \mathrm{CM}: \quad 1.63 \quad 62.7$

RHO PHI

RHO PHI

$\begin{array}{ll}1.76 & 54.6 \\ 1.72 & 57.3\end{array}$

$90.0 \mathrm{CM}: \quad 1.6959 .1$

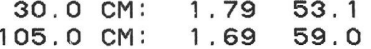

SECTI ON AVERAGE:

TOP OF SECTION 156.80 METERS SUB-BOTTOM

$\begin{array}{rrrr}0.0 & \text { CM: } & \text { RHO } & \text { PHI } \\ 75.0 & 1.57 & 66.5 \\ 75: & 1.71 & 58.2\end{array}$

15.0 cM: $\quad \begin{array}{r}\text { RHO } \\ \text { PHI }\end{array}$

RHO $\mathrm{PHI}$

$\begin{array}{lll}75.0 \mathrm{CM}: & 1.71 & 58.2 \\ \text { AVERAGE: } & 1.63 & 63.0\end{array}$

90.0 CM: 1.71 57.9

$\begin{array}{lll}105.0 \mathrm{CM} & 1.51 & 70.3\end{array}$

TOP OF SECTION

158.30 METERS SUB-BOTTOM

$386 \quad 52$

$\begin{array}{lr}\mathrm{RHO} & \mathrm{PHI} \\ .71 & 57.8\end{array}$

15.0 CM: 1.75 R5.

30.0 CM: $\begin{array}{rr}\text { RHO } & \text { PHI } \\ 1.80 & 52.4\end{array}$

$\begin{array}{rlll}0.0 & C M: & 1.71 & 57.8 \\ 75.0 & C M: & 1.92 & 45.1\end{array}$

.0 2.0139 .5

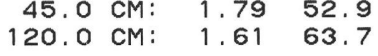
SECTION AVERAGE: 1.8052 .1
45. O CM: $\quad{ }^{\mathrm{RHO}} \quad \mathrm{PH}$ 120.0 CM: 1.5169 .9

RHO PHI $\begin{array}{rrr}45.0 \mathrm{CM}: & 1.57 & 66.3 \\ 120.0 \mathrm{CM}: & 1.57 & 66.3\end{array}$

RHO PHI 120.0 CM: 1.58 65.8

45. $\mathrm{CM}:$ RHO $\mathrm{PH}$ 45.

$45.0 \mathrm{cM}: \quad \mathrm{RHO} \quad \mathrm{PHI}$

$\begin{array}{rll}\text { 45.0 CM: } & 1.60 & 64.5 \\ 120.0 \mathrm{CM}: & 1.61 & 64.1\end{array}$

RHO PHI

$\begin{array}{rrr}45.0 \mathrm{CM}: & 1.63 & 62.5 \\ 120.0 \mathrm{CM}: & 1.68 & 59.6\end{array}$

45. O CM: RHO PHI $\begin{array}{rrr}45.0 \mathrm{CM}: & 1.70 & 58.2 \\ 120.0 \mathrm{CM}: & 1.59 & 65.4\end{array}$

45.0 CM: $\begin{array}{rr}\text { RHO } & \text { PHI } \\ 1.79 & 52.9\end{array}$

45.0 CM: $\begin{array}{rr}\text { RHO } & \text { PHI } \\ 1.52 & 69.6\end{array}$

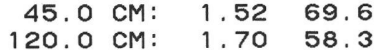

$0>[2.65 / 6.61 \mathrm{C} 0 / 1.5 / 4]$ 60.0 CM: $\begin{array}{rr}\mathrm{RHO} & \mathrm{PHI} \\ 1.57 & 66.2\end{array}$ 135.0 CM: $1.47 \quad 72.4$

$0>[2,65 / 6,61 \mathrm{C} 0 / 1,5 / 4]$ $60.0 \mathrm{CM}: \quad \begin{array}{r}\mathrm{RHO} \\ \quad \mathrm{PHI}\end{array}$ $135.0 \mathrm{CM}: 0.00 \quad 0.0$

$0>[2.65 / 6.61 \mathrm{C} 0 / 1.5 / 4]$ $60.0 \mathrm{cM}: \quad \begin{array}{rr}\mathrm{RHO} & \mathrm{PHI} \\ 1.60 & 64.5\end{array}$ 135.0 CM: 1.57 66.2

$0>[2.65 / 6.61 \mathrm{C} \quad 0 / 1.5 / 4]$

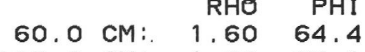
135. O CM: 1.6263 .1

$0>[2.65 / 6.61 \mathrm{C} 0 / 1.5 / 4]$ $\begin{array}{rrr}60.0 \mathrm{CM}: & \text { RHO } & \mathrm{PHI} \\ 1.59 & 65.3\end{array}$ $135.0 \mathrm{CM}: 1.5965 .1$

$0>[2.65 / 6.61 \mathrm{C} \quad 0 / 1.5 / 4]$ $60.0 \mathrm{cM}: \quad \mathrm{RHO} \mathrm{PHI}$ $135.0 \mathrm{CM}: 1.5866 .0$

$0>[2.65 / 6.61 \mathrm{C} 0 / 1.5 / 4]$

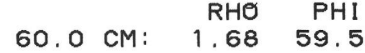
$135.0 \mathrm{CM}: 0.00 \mathrm{0}$

$0>[2.65 / 6.61 \mathrm{C} 0 / 1.5 / 4]$ 60.0 CM: $\begin{array}{rrr}\text { RHO } & \text { PHI } \\ 1.78 & 53.8\end{array}$ 135.0 CM: 1.6561 .2

$0>[2.65 / 6.61 \mathrm{C} 0 / 1,5 / 4]$

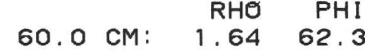
$35.0 \mathrm{~cm}: \quad 1.70 \mathrm{58.2}$

$0>[2.65 / 6.61 \mathrm{C} \quad 0 / 1.5 / 4]$

45. 0 CM: RHO PHI

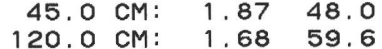

60.0 CM: $1.88 \quad 47.5$ 
$386 \quad 6$$$
62
$$

$0.0 \mathrm{~cm}$

SECTION TV.O CM:

$386 \quad 63$

$\begin{array}{llll}\text { RHO OF SECTION 169.30 METERS SUB-BOTTOM } \\ 0.0 \mathrm{CM}: & 1.53 & \text { PHI } & \text { RHO PHI }\end{array}$

$\begin{array}{lllllllll}\text { RHO } & \text { PHI }\end{array}$ SECTION AVERAGE: 1.58 66.1

$386 \quad 64$

TOP OF SECTION 170.80 METERS SUB-BOTTOM

O. $0 \mathrm{CM}$

RHO $\mathrm{PHI}$

$\begin{array}{ll}1.54 & 68.5 \\ 2.05 & 37.2\end{array}$

15.0 CM: $\begin{array}{rrr}\text { RHO } & \text { PHI } \\ 1.61 & 64.2\end{array}$

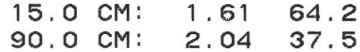

30. $\mathrm{CM}: \quad \mathrm{RHO} \mathrm{PHI}$

$\begin{array}{rrr}105.0 \mathrm{CM}: & 2.01 & 61.3 \\ & & 39.7\end{array}$

SECTION AVERAGE:

TOP OF SECTION 177.30 METERS SUB-BOTTOM

$386 \quad 72$

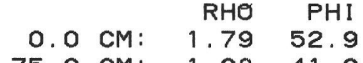

$15.0 \mathrm{CM}: \quad \begin{array}{r}\mathrm{RHO} \\ 1.85\end{array}$

$\begin{array}{lll}75.0 \mathrm{CM}: & 1.98 & 41.3 \\ \text { AVERAGE: } & 1.92 & 45.0\end{array}$

90.0 CM: $\quad 1.97 \quad 41.8$

30. $\mathrm{CM}: \mathrm{RHO} \mathrm{PHI}$

38673

TOP OF SECTION 178.80 METERS SUB-BOTTOM

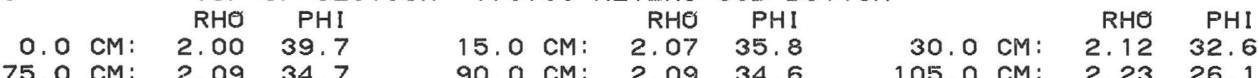

SECTION AVERAGE: 2.0636 .5

TOP OF SECTION 186.80 METERS SUB-BOTTOM

$\begin{array}{lll}386 & 82\end{array}$

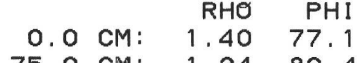

15.0 CM: $\quad 1.50 \quad 71.0$

30. O CM: $\quad$\begin{tabular}{rr} 
RHO & PHI \\
\hline & 70.50
\end{tabular}

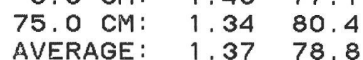

SECTI ON AVERAGE:

$386 \quad 83$

TOP OF SECTION 188.30 METERS SUB-BOTTOM

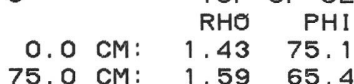

$\begin{array}{rrr} & \text { RHO } & \text { PHI } \\ 15.0 \mathrm{CM}: & 1.32 & 81.7\end{array}$

$\begin{array}{rr}105.0 \mathrm{CM}: & 1.22 \\ & 87.9\end{array}$

SECTION AVERAGE: 1.52 69.3

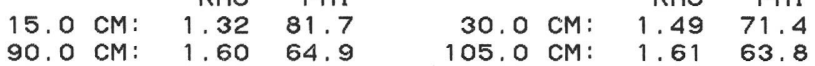

$386 \quad 86$

TOP OF SECTION 192.80 METERS SUB-BOTTOM

$\begin{array}{rrr}0.0 \mathrm{CM}: & \mathrm{RHO} & \mathrm{PHI} \\ 75.0 \mathrm{CM} & 0.00 & 0.0 \\ 1.85 & 48.9\end{array}$

$\begin{array}{lll} & \\ \text { SECTION AVERAGE: } & 1.82 & 50.9\end{array}$

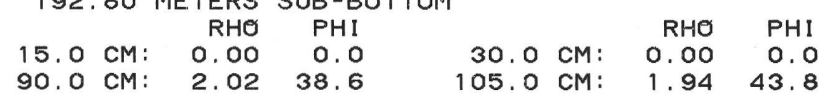

$105.0 \mathrm{CM}: \quad 1.94 \quad 43.8$
$45.0 \mathrm{cM}:$ RHO PHI

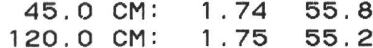

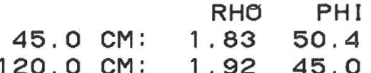

RHO $\mathrm{PHI}$

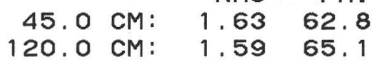

45. PHI $\begin{array}{rrr}45.0 \mathrm{CM}: & 1.59 & 65.3 \\ 120.0 \mathrm{CM}: & 1.58 & 65.7\end{array}$

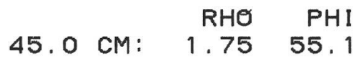
120.0 CM: 1.9046 .2

45.0 $\mathrm{RHI}$

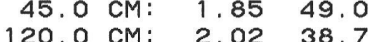

45. 0 cM: $2.15 \quad 30.7$ $120.0 \mathrm{CM}: 2.02 \quad 39.1$

45.0 CM: $\begin{array}{rrr}\text { RHO } & \text { PHI } \\ 1.53 & 69.2\end{array}$ $120.0 \mathrm{CM}: 1.26 \quad 85.4$

45.0 cM: $\quad \begin{array}{rr}\text { RHO } & \text { PHI } \\ 1.53 & 69.2\end{array}$ $120.0 \mathrm{CM}: 1.56 \quad 67.3$

45. 0 cM: $\begin{array}{rr}\text { RHO } & \text { PHI } \\ 1.31 & 82.3\end{array}$

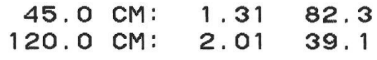

$0>[2,65 / 6,61 \mathrm{C} \quad 0 / 1,5 / 4]$ 60.0 CM: $1.75 \quad 55$. $135.0 \mathrm{CM}: \quad 1.76 \quad 54.5$

$0>[2.65 / 6,61 \mathrm{C} 0 / 1,5 / 4]$ 60.0 CM: 1.7257 .0 $135.0 \mathrm{CM}: 1.88 \quad 47.5$

$0>[2.65 / 6.61 \mathrm{C} \quad 0 / 1.5 / 4]$ 60.0 CM: $\begin{array}{rr}\mathrm{RHO} & \mathrm{PHI} \\ 1.64 & 62.3\end{array}$ $135.0 \mathrm{CM}: \quad 1.57 \quad 66.6$

$0>[2.65 / 6.61 \mathrm{C} \quad 0 / 1.5 / 4]$ $60.0 \mathrm{CM}: \quad \begin{array}{rr}\mathrm{RHO} & \mathrm{PHI} \\ 1.60 & 64.5\end{array}$ 35.0 CM: 1.6064 .9

$0>[2.65 / 6.61 \mathrm{C} 0 / 1.5 / 4]$ $60.0 \mathrm{~cm}: \quad \begin{array}{rr}\mathrm{RHO} & \mathrm{PHI} \\ 1.98 & 41.0\end{array}$ 135.0 CM: 1.9046 .3

$0>[2.65 / 6.61 \mathrm{C} 0 / 1.5 / 4]$ $\begin{array}{rrr}60.0 \mathrm{CM}: & \begin{array}{r}\mathrm{RHO} \\ 1.98\end{array} & 41.0 \\ 135.0 \mathrm{CM}: & 1.88 & 47.7\end{array}$

$0>[2.65 / 6.61 \mathrm{C} 0 / 1.5 / 4]$ $\begin{array}{rrr}60.0 \mathrm{CM}: & 2.10 & 33.8 \\ 135.0 \mathrm{CM}: & 1.63 & 62.7\end{array}$

$0>[2.65 / 6.61 \mathrm{C} 0 / 1.5 / 4]$ 60.0 CM: $\begin{array}{rrr}\text { RHO } & \text { PHI } \\ 1.40 & 76.7\end{array}$ $135.0 \mathrm{CM}: 1.3977 .8$

$0>[2.65 / 6.61 \mathrm{C} \quad 0 / 1.5 / 4]$ $60.0 \mathrm{cM}: \quad \begin{array}{r}\mathrm{RHO} \\ 1.62\end{array}$ 135.0 CM: $0.00 \quad 0.0$

$0>[2.65 / 6.61 \mathrm{C} \quad 0 / 1.5 / 4]$ $\begin{array}{rrr}60.0 \mathrm{CM}: & 1.49 & 71.3 \\ 135.0 \mathrm{CM}: & 1.50 & 70.6\end{array}$ 
TOP OF SECTION 207.30 METERS SUB-BOTTOM

$0.0 \mathrm{~cm}$

75. 0 CM:

$1.50 \quad 71.0$

RHO PHI

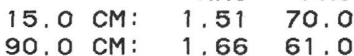

$\begin{array}{rrr}30.0 \mathrm{CM}: & 1.57 & \mathrm{PHI} \\ 105.0 \mathrm{CM}: & 1.82 & 51.2\end{array}$

SECTION AVERAGE

$386 \quad 112$

CM: RHO PHI RHO PHI RHO PHI

$65.9 \quad 15.0 \mathrm{CM}: 1.5468 .5 \quad 30.0 \mathrm{CM}: 1.5468 .1$

SECTION AVERAGE: 1.5667 .2

$386 \quad 122$

$0.0 \mathrm{CM}$ $75.0 \mathrm{CM}$

SECTI ON AVERAGE:

$386 \quad 123$

TOP OF SECTION 283.40 METERS SUB-BOTTOM

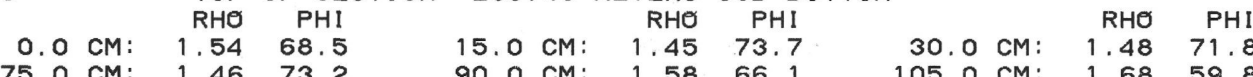

SECTION AVERAGE: 1.56 67.0

$386 \quad 124$

TOP OF SECTION 284.90 METERS SUB-BOTTOM

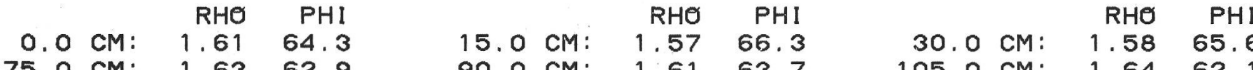

$\begin{array}{lll}75.0 \mathrm{CM}: & 1.63 & 62.9 \\ \text { SECTION AVERAGE: } & 1.62 & 63.6\end{array}$

$386 \quad 132$

TOP OF SECTION 310.50 METERS SUB-BOTTOM

$0.0 \mathrm{CM}: \quad \begin{array}{rr}\mathrm{RHO} & \mathrm{PHI} \\ 0.40 & 77.1\end{array}$

15.0 CM: RHO PHI

$\begin{array}{lll}\text { TS.O CM: } & 1.61 & 64.1 \\ \text { AVERAGE: } & 1.71 & 57.8\end{array}$

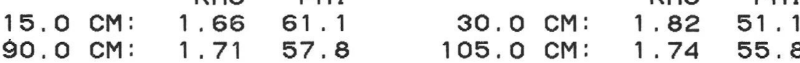

SECTION AVERAGE:

$386 \quad 133$

TOP OF SECTION $\mathbf{3 1 2 . 0 0}$ METERS SUB-BOTTOM

$\begin{array}{rrr}0.0 \mathrm{CM}: & \mathrm{RHO} & \mathrm{PHI} \\ 75.62 & 63.3 \\ & 1.81 & 51.6\end{array}$

15.0 CM: 1.68 PHO PHI

$\begin{array}{lll}\text { 75. O CM: } & 1.81 & 51.6 \\ \text { AVERAGE: } & 1.77 & 54.3\end{array}$

$90.0 \mathrm{CM}: \quad 1.82 \quad 51.1$

30. $\mathrm{CM}: \quad \mathrm{RHO} \quad \mathrm{PHI}$

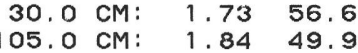

SECTION AVERAGE

$386 \quad 14 \quad 1$

O. $0 \mathrm{CM}$

TOP OF SECTION 328.50 METERS SUB-BOTTOM

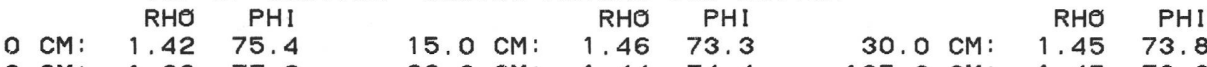

15.0 CM: $1.44 \quad 74.4 \quad 105.0 \mathrm{cM}: 1.45 \quad 73.8$

$386 \quad 142$

TOP OF SECTION 330.00 METERS SUB-BOTTOM

$0.0 \mathrm{CM}$

$\mathrm{RHO} \mathrm{PHI}$

$\begin{array}{ll}1.43 & 75.2 \\ 1.45 & 73.6\end{array}$

15.0 CM: $\quad \begin{array}{rr}\text { RHO } & \text { PHI } \\ 1.45 & 74.1\end{array}$

30.0 $\mathrm{CM}:$ RHO PHI

SECTION AVERAGE:

$386 \quad 143$

TOP OF SECTION 331.50 METERS SUB-BOTTOM

$0.0 \mathrm{~cm}: \quad \mathrm{RHO} \mathrm{PHI} \quad 15 \mathrm{~cm}$ RHO $\mathrm{PHI}$ RHO PHI

$\begin{array}{rlllllrlrl}0.0 & \mathrm{CM}: & 1.42 & 75.6 & 15.0 \mathrm{CM}: & 1.45 & 73.9 & 30.0 \mathrm{CM}: & 1.45 & 73.9 \\ 75.0 & \mathrm{CM}: & 1.54 & 68.1 & 90.0 \mathrm{CM}: & 1.48 & 72.0 & 105.0 \mathrm{CM}: & 1.47 & 72.7\end{array}$

SECTION AVERAGE: 1.4971 .2
45.0 CM: $\quad \begin{array}{rrr}\text { RHO } & \text { PHI } \\ 1.62 & 63.5\end{array}$ 120.0 CM: $1.83 \quad 50.5$

45. O CM: $\quad 1.59$ RHO 65.0 120.0 CM: 1.5667 .4

45. $\mathrm{CM}: \mathrm{RHO} \quad \mathrm{PHI}$ 45. $\mathrm{CM:} \quad 1.4176$.

RHO PHI 45. O CM: 1.60 64.9

RHO PHI $\begin{array}{rll}45.0 \mathrm{CM}: & 1.63 & 62.7 \\ 120.0 \mathrm{CM}: & 1.68 & 59.5\end{array}$

25HO PHI

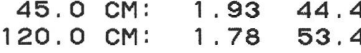

45. O CM: $\quad \begin{array}{rr}\text { RHO } & \mathrm{PHI} \\ 56.73 & \end{array}$

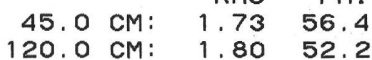

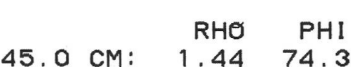
$\begin{array}{rrr}\text { 45.0 CM: } & 1.44 & 74.3 \\ 120.0 \mathrm{CM}: & 1.41 & 76.4\end{array}$ $120.0 \mathrm{~cm}$

45. $\mathrm{RHO} \mathrm{PHI}$ 45. $0 \mathrm{CM}: 1.45$ 73.9

45. $\mathrm{RHO} \mathrm{PHI}$ $\begin{array}{rrr}45.0 \mathrm{CM}: & 1.54 & 68.5 \\ 120.0 \mathrm{CM}: & 1.51 & 70.1\end{array}$
$0>[2.65 / 6.61 \mathrm{C} 0 / 1.5 / 4]$ $60.0 \mathrm{cM}: \quad$ RHO $\mathrm{PHI}$ $135.0 \mathrm{CM}: 1.8548 .9$

$0>[2.65 / 6.61 \mathrm{C} 0 / 1.5 / 4]$

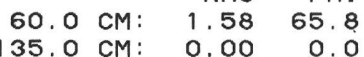

$0>[2.65 / 6.61 \mathrm{C} 0 / 1.5 / 4]$ 60.0 CM: 1.3778 .7

$0>[2.65 / 6.61 \mathrm{C} 0 / 1.5 / 4]$ $60.0 \mathrm{cM}: \quad \mathrm{RHO} \mathrm{PHI}$ $135.0 \mathrm{CM}: \quad 1.69$ 59.0

$0>[2.65 / 6.61 \mathrm{C} \quad 0 / 1.5 / 4]$

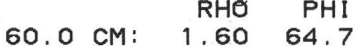
$135.0 \mathrm{CM}: 1.6064 .6$

$0>[2.65 / 6.61 \mathrm{C} 0 / 1.5 / 4]$

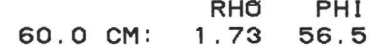
135.0 CM: $1.72 \quad 57.3$

$0>[2.65 / 6.61 \mathrm{C} \quad 0 / 1.5 / 4]$ $60.0 \mathrm{cM}: \quad \begin{array}{rr}\mathrm{RHO} & \mathrm{PHI} \\ 1.70 & 58.5\end{array}$ $135.0 \mathrm{CM}: 1.95 \quad 42.8$

$0>[2.65 / 6.61 \mathrm{C} \quad 0 / 1.5 / 4]$

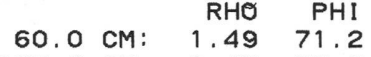
135.0 CM: 1.4573 .9

$0>[2.65 / 6.61 \mathrm{C} \quad 0 / 1.5 / 4]$ 60.0 CM: $1.44 \quad 74.2$ $[0.0 \%$ RHO PHI 135.0 CM: 1.5368 .6 


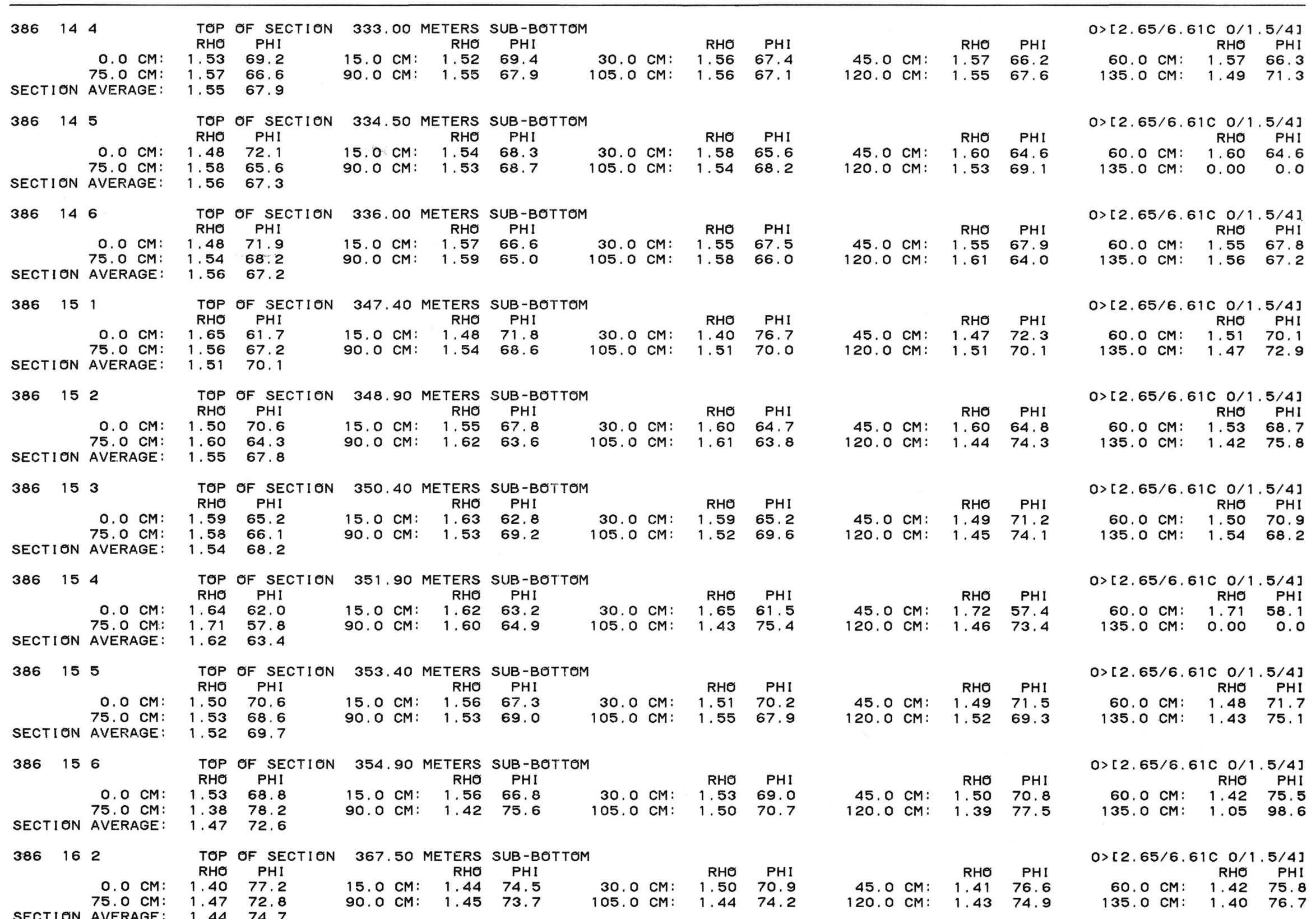


$386 \quad 163$

TOP OF SECTION 369.00 METERS SUB-BOTTOM

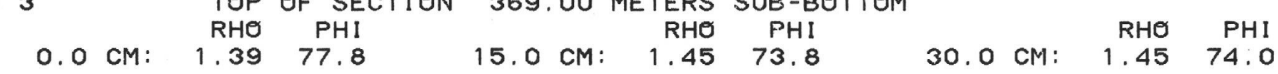

CM: $\quad 1.46 \quad 73.5$

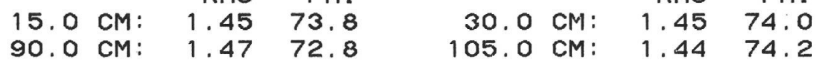

SECTION AVERAGE: 1.4871 .7

$386 \quad 16 \quad 4$

TOP OF SECTION 370.50 METERS SUB-BOTTOM

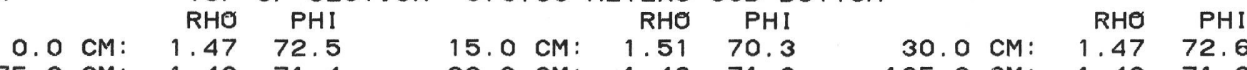

SECTION AVERAGE: 1.4971 .4

$386 \quad 16 \quad 5$

TOP OF SECTION 372.00 METERS SUB-BOTTOM

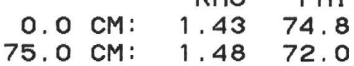

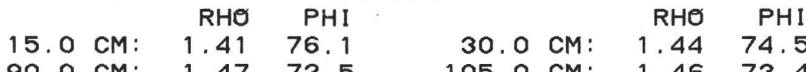

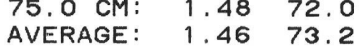

$386 \quad 171$

TOP OF SECTION $\mathbf{3 8 5 . 5 0}$ METERS SUB-BOTTOM

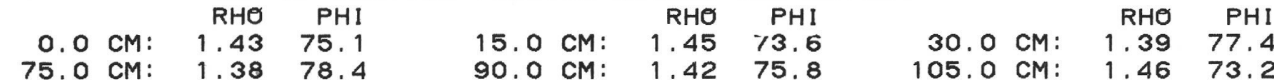

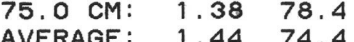

SECTION AVERAGE

$\begin{array}{lll}386 & 172\end{array}$

'o. C CM: \begin{tabular}{rr} 
RHO & $\mathrm{PHI}$ \\
\hline .78 & 53.7
\end{tabular}

75.0 CM: $\quad 1.4574 .0$

SECTION AVERAGE: 1.5667 .1

$\begin{array}{lll}386 & 173\end{array}$

\section{TOP OF SECTION 388.50 METERS SUB-BOTTOM}

$\begin{array}{rrr}0.0 \mathrm{CM}: & \mathrm{RHO} & \mathrm{PHI} \\ & 1.53 & 68.7\end{array}$

75.0 CM: 1.5269 .6

SECTION AVERAGE: 1.5369 .1

$\begin{array}{rrrrrr}15.0 \mathrm{CM}: & 1.55 & 67.7 & 30.0 \mathrm{CM}: & 1.52 & 69.4 \\ 90.0 \mathrm{CM}: & 1.54 & 68.4 & 105.0 \mathrm{CM}: & 1.54 & 68.4\end{array}$

$386 \quad 174$

TOP OF SECTION 390.00 METERS SUB-BOTTOM

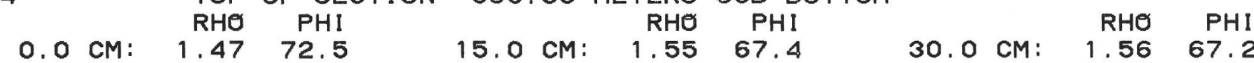

$\begin{array}{llllrlll}0.0 & & & \end{array}$

SECTION AVERAGE: 1.53 68.7

$386 \quad 175$

TOP OF SECTION 391.50 METERS SUB-BOTTOM

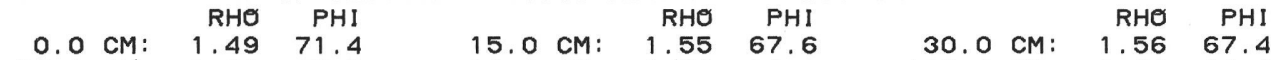

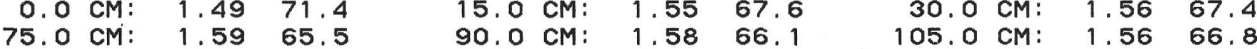

SECTION AVERAGE: 1.55 67.6

$386 \quad 176$

TOP OF SECTION 393.00 METERS SUB-BOTTOM

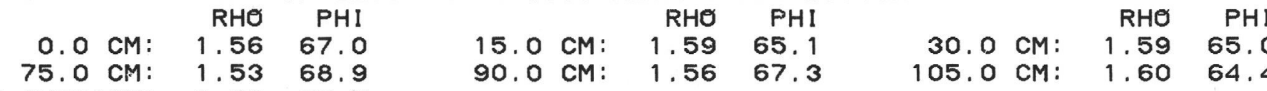

SECTION AVERAGE: 1.5368 .9

$386 \quad 18$

TOP OF SECTION 404.15 METERS SUB-BOTTOM

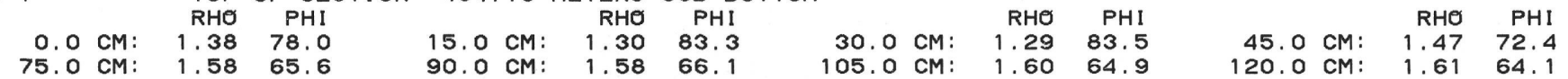

$0>[2.65 / 6.61 \mathrm{C} 0 / 1.5 / 4]$ $60.0 \mathrm{cM}: \quad 1.46 \quad 73.5$ $135.0 \mathrm{CM}: 1.62 \quad 63.4$

45. O CM: RHO 72 $120.0 \mathrm{CM}: 1.66$ 60.9

45. 0 CM: $\quad \begin{array}{rr}\text { RHO } & \text { PHI } \\ 1.48 & 72.0\end{array}$

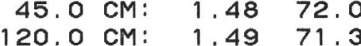

$0>[2.65 / 6.61 \mathrm{C} 0 / 1.5 / 4]$

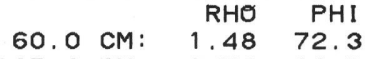
$135.0 \mathrm{CM}: \quad 1.5269 .5$

$0>[2.65 / 6.61 \mathrm{C} 0 / 1.5 / 4]$ $60.0 \mathrm{cM}: \quad \begin{array}{rr}\mathrm{RHO} & \mathrm{PHI} \\ 1.46 & 73.2\end{array}$ 135.0 CM: 1.5071 .0

$0>[2.65 / 6.61 \mathrm{C} 0 / 1.5 / 4]$ $\begin{array}{rrr}60.0 \mathrm{CM}: & \text { RHO } & \text { PHI } \\ 1.39 & 77.7\end{array}$ $135.0 \mathrm{CM}: \quad 1.70 \quad 58.2$

$0>[2.65 / 6.61 \mathrm{C} 0 / 1.5 / 4]$ $60.0 \mathrm{CM}: \quad$ RHO PHI $135.0 \mathrm{CM}: \quad 1.5169 .9$

$0>[2.65 / 6.61 \mathrm{C} 0 / 1.5 / 4]$ $60.0 \mathrm{cM}: \quad$ RHO 5 PHI

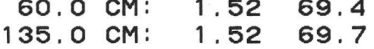

45. O CM: 1.4971. $120.0 \mathrm{CM}: 1.5468$.

45. O CM: $\quad \begin{array}{r}\text { RHO } \\ \text { PHI }\end{array}$ $\begin{array}{rrr}45.0 \mathrm{CM}: & 1.54 & 68.4 \\ 120.0 \mathrm{CM}: & 1.53 & 68.6\end{array}$

$0>[2.65 / 6.61 \mathrm{C} 0 / 1.5 / 4]$ $60.0 \mathrm{cM}: \quad \begin{array}{r}\mathrm{RHO} \\ \mathrm{PHI}\end{array}$ 135.0 CM: 1.50 70.6

$0>[2.65 / 6.61 \mathrm{C} 0 / 1.5 / 4]$ $60.0 \mathrm{cM}: \quad \begin{array}{rr}\text { RHO } & \mathrm{PHI} \\ 1.52 & 69.3\end{array}$ $135.0 \mathrm{CM}: 1.5567 .6$

$0>[2.65 / 6.61 \mathrm{C} 0 / 1.5 / 4]$ $60.0 \mathrm{cM}: \quad \begin{array}{rr}\mathrm{RHO} & \mathrm{PHI} \\ 1.56 & 67.4\end{array}$ $0>[2.65 / 6.61 \mathrm{C} 0 / 1.5 / 4]$ RHO PHI $135.0 \mathrm{CM}: 1.5567 .8$

SECTION AVERAGE: 1.5170 .3 
$\begin{array}{lll}386 & 18 \quad 4\end{array}$

TOP OF SECTION 408.65 METERS SUB-BOTTOM

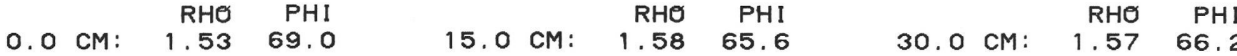

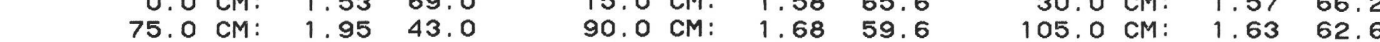
SECTION AVERAGE: 1.64 62.4

$386 \quad 18 \quad 5$

\section{TOP OF SECTION 410.15 METERS SUB-BOTTOM}

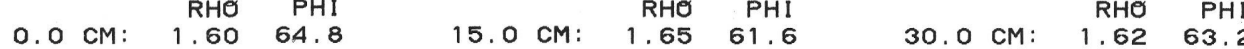

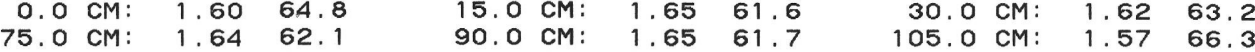

SECTION AVERAGE: 1.6263.
TOP OF SECTION 411.65 METERS SUB-BOTTOM

$\begin{array}{rrrr}0.0 & \text { CM: } & \text { RHO } & \text { PHI } \\ 75.0 & \text { CM: } & 1.77 & 77.0 \\ 73.9\end{array}$

$\begin{array}{lll}75.0 \mathrm{CM}: & 1.77 & 53.9 \\ \text { AVERAGE: } & 1.72 & 57.3\end{array}$

SECTIION AVERAGE:

$386 \quad 19$

TOP OF SECTION 415.10 METERS SUB-BOTTOM

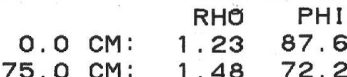

SECTION AVERAGE: $1.55 \quad 67.8$

$386 \quad 193$

TOP OF SECTION 416.60 METERS SUB-BOTTOM

O.O CM:

$\mathrm{RHO} \mathrm{PH}$

75.0 CM: $\quad 1.6263 .5$

$15.0 \mathrm{cM}: \quad \begin{array}{rrr}\mathrm{RHO} & \mathrm{PHI} \\ & 1.68 & 59.5\end{array}$

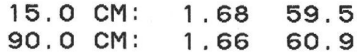

30.0 CM: $\quad 1.64 \quad 62.2$

SECTION AVERAGE:

TOP OF SECTION 424.60 METERS SUB-BOTTOM

$386 \quad 202$

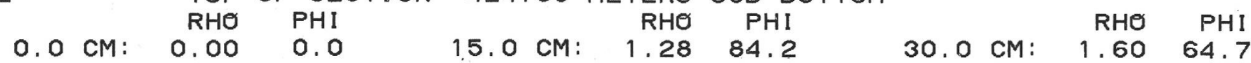

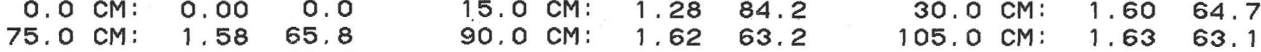

SECTION AVERAGE: 1.5766 .4

386203

TOP OF SECTION 426.10 METERS SUB-BOTTOM

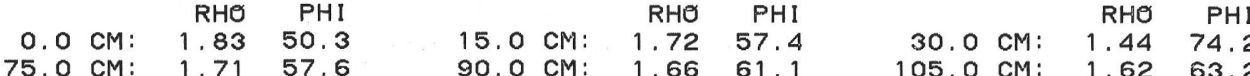

SECTION AVERAGE: $\quad 1.6064 .5$

386204

TOP OF SECTION 427.60 METERS SUB-BOTTOM

$\begin{array}{rrr}0.0 \mathrm{CM}: & 1.57 & \mathrm{RHI} \\ 75.0 \mathrm{CM}: & 1.55 & 67.8\end{array}$

15. CM: RHO PHI 158 RHO PHI

$90.0 \mathrm{CM}: \quad 1.61$ 64.3

105. $\mathrm{CM}: \quad 1.5965 .3$
45.0 CM: $\begin{array}{rrr}\text { RHO } & \text { PHI } \\ 1.62 & 63.6\end{array}$

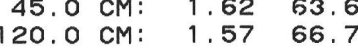

$45.0 \mathrm{CM}: 1.50 \mathrm{PHI}$ $120.0 \mathrm{CM}: 1.606564$

RHO PHI 45. $\mathrm{CM}: \quad 1.5766 .2$ RHO PHI

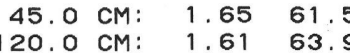
45.0 cM: RHO PHI $120.0 \mathrm{CM}: 1.73 \quad 56.5$

RHO PHI

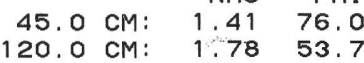

RHO PHI $\begin{array}{rll}\text { 45.0 CM: } & 1.57 & 66.2 \\ 120.0 \mathrm{CM}: & 1.65 & 61.7\end{array}$

RHO PHI 45.0 CM: $1.64 \quad 62.2$ RHO PHI 45.0 CM: 1.3977 .7

RHO PHI

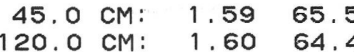

$0>[2.65 / 6,61 \mathrm{C} 0 / 1.5 / 4]$ $60.0 \mathrm{CM}: 1.6263$. . 1.5567.

$0>[2.65 / 6.61 \mathrm{C} 0 / 1.5 / 4]$ $60.0 \mathrm{cM}: \quad{ }^{\mathrm{RHO}} \mathrm{rH}$ 135. CM: 1.5965.

$0>[2.65 / 6.61 \mathrm{C} 0 / 1.5 / 4]$ $60.0 \mathrm{CM}: 1.5965$. 135. 0 CM: 1.5766 .3

$0>[2.65 / 6.61 \mathrm{C} 0 / 1.5 / 4]$ $\begin{array}{rrr}60.0 \mathrm{cM}: & 1.61 & 63.8 \\ 135.0 \mathrm{cM}: & 0.00 & 0.0\end{array}$

$0>[2.65 / 6.61 \mathrm{C} 0 / 1.5 / 4]$ 60.0 CM: $\quad 1.77 \quad 54$ $135.0 \mathrm{CM}: 1.7555 .3$

$0>[2.65 / 6.61 \mathrm{C} 0 / 1.5 / 4$ $60.0 \mathrm{cM}: \quad \begin{array}{r}\mathrm{RHO} \\ \mathrm{PHI}\end{array}$ $135.0 \mathrm{CM}: \quad 1.83 \quad 50.5$

$0>[2.65 / 6.61 \mathrm{C} 0 / 1.5 / 4]$ $60.0 \mathrm{cM}: \quad \begin{array}{rr}\mathrm{RHO} & \mathrm{PHI} \\ 1.58 & 65.7\end{array}$ 135.0 CM: 1.4772 .5

$0>[2.65 / 6.61 \mathrm{C} 0 / 1.5 / 4]$ $60.0 \mathrm{CM}: \quad \begin{array}{rr}\mathrm{RHO} & \mathrm{PHI} \\ 1.57 & 66.7\end{array}$ 135. O CM: $1.49 \quad 71.4$

$0>[2.65 / 6.61 \mathrm{C} 0 / 1.5 / 4]$ 60.0 CM: $\quad$ RHO PHI 135. O CM: 1.60 64.9

$0>[2.65 / 6.61 \mathrm{C} \quad 0 / 1.5 / 4]$ 60.0 CM: $\quad \begin{array}{rl}\mathrm{RHO} & \mathrm{PH} \\ 1.59 & 65.5\end{array}$ $135.0 \mathrm{cM}: \quad 1.57 \quad 66.3$ SECTION AVERAGE: 1.5865 .6 


\section{O. $0 \mathrm{CM}:$ \\ SECTION AVERAGE:}

$\begin{array}{lll}386 & 21 \quad 3\end{array}$

$0.0 \mathrm{CM}$
$75.0 \mathrm{CM}$ SECTION AVERAGE:

$386 \quad 21 \quad 4$

TOP OF SECTION

$75.0 \mathrm{CM}$

$\begin{array}{lll}386 & 21 \quad 5\end{array}$

O.O CM:

SECTI ON AV.O CM:

386216

$\begin{array}{llll} & \text { TOP OF SECTION } & 440.20 & \text { METERS SUB-BOTTOM } \\ 0.0 \mathrm{CM}: & \text { RHO PHI } & & \end{array}$

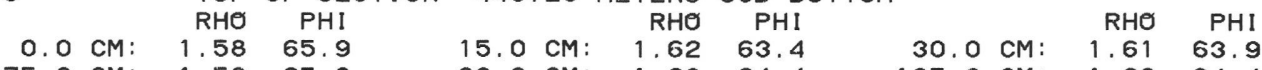

$\begin{array}{rll}75.0 \mathrm{CM}: & 1.59 & 65.3 \\ \text { SECTION AVERAGE: } & 1.60 & 64.8\end{array}$

386222

TOP OF SECTION 443.60 METERS SUB-BOTTOM

\begin{tabular}{rrr}
0.0 CM: & RHO & PHI \\
75.50 & 70.8 \\
\hline & 1.58 & 66.1
\end{tabular}

$\begin{array}{rrr}15.0 \mathrm{CM}: \quad 1.60 & 64.5\end{array}$

90.0 CM: $\quad 1.55 \quad 67.8$

30.0 CM: $\begin{array}{rr}\mathrm{RHO} & \mathrm{PHI} \\ 1.57 & 66.4\end{array}$

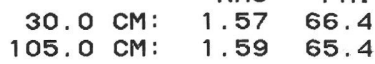
SECTION AVERAGE: 1.5766 .6

$386 \quad 22 \quad 3$

\section{TOP OF SECTION 445.10 METERS SUB-BOTTOM}

RHO PHI RHO PHI RHO PHI $0.0 \mathrm{CM}:$

SECTI ON AVERAGE:

386224

$1.56 \quad 66.9$

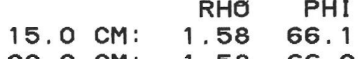

$\begin{array}{rrr}30.0 \mathrm{CM}: & \text { RHO } & \text { PHI } \\ 105.0 \mathrm{CM}: & 1.54 & 68.3 \\ & 1.56 .3\end{array}$

TOP OF SECTION 446.60 METERS SUB-BOTTOM

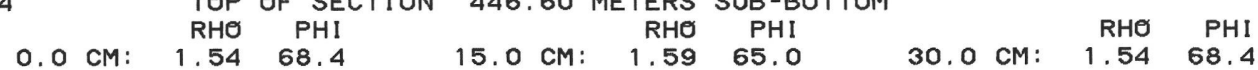

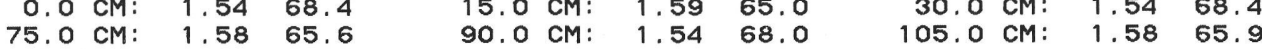

SECTION AVERAGE: 1.62 63.6

TOP OF SECTION 448.10 METERS SUB-BOTTOM

$386 \quad 225$

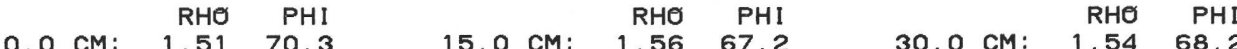

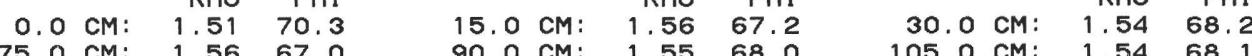

SECTION AVERAGE: 1.5567 .9

TOP OF SECTION 453.20 METERS SUB-BOTTOM

$\begin{array}{lll}386 & 23 \quad 2\end{array}$

\begin{tabular}{rrr}
$0.0 \mathrm{CM}:$ & RHO & PHI \\
$75.0 \mathrm{CM}:$ & 1.65 & 65.4 \\
\hline AVERAG & 1.61 .5
\end{tabular}

15.0 CM: $\quad \begin{array}{rr}\text { RHO } & \text { PHI } \\ 1.45 & 73.8\end{array}$

30. 0 CM: $\quad 1.50$ RHO 70.5

$90.0 \mathrm{CM}: 1.64 \quad 62.3$

$105.0 \mathrm{CM}: 1.63 \quad 62.9$
45.0 CM: $\begin{array}{rr}\text { RHO } & \text { PHI } \\ 1.67 & 60.6\end{array}$ $120.0 \mathrm{CM}: 1.34$ 80.6

45. 0 CM: $\quad \begin{array}{r}\mathrm{RHO} \\ \mathrm{PHI}\end{array}$ $\begin{array}{rrr}45.0 \mathrm{CM}: & 1.65 & 61.7 \\ 120.0 \mathrm{CM}: & 1.64 & 62.4\end{array}$

45. O CM: $\quad \begin{array}{r}\text { RHO } \\ \text { PHI }\end{array}$ $\begin{array}{rll}45.0 \mathrm{CM}: & 1.62 & 63.6 \\ 120.0 \mathrm{CM}: & 1.62 & 63.3\end{array}$

45. $\mathrm{CM}: \begin{array}{lll}\text { RHO } & \mathrm{PHI} \\ 0.00 & 0.0\end{array}$ $120.0 \mathrm{CM}: 1.6164$.

45. 0 CM: $\quad$ RHO PHI

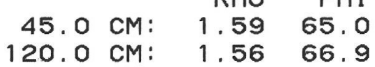

RHO PHI

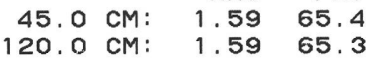

45. $\mathrm{CM}$ RHO PHI

45.0 CM: 1.5866 .0

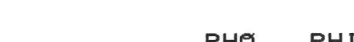

45.0 cM: $2.07 \quad \begin{aligned} \text { PHI } \\ \text { PHI }\end{aligned}$

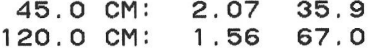

RHO PHI

$\begin{array}{rrr}45.0 \mathrm{CM}: & 1.55 & 67.4 \\ 120.0 \mathrm{CM}: & 1.54 & 68.2\end{array}$

45.0 CM: $\begin{array}{rr}\text { RHO } & \text { PHI } \\ 1.67 & 60.4\end{array}$

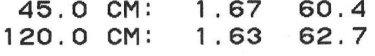

O) $[2.65 / 6.61 \mathrm{C} 0 / 1.5 / 4]$ 60.0 CM: 1.6362 .5 $135.0 \mathrm{CM}$ : $1.55 \mathrm{~s}$. 67.8

$0>[2.65 / 6.61 \mathrm{C} 0 / 1.5 / 4]$

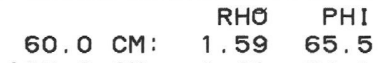

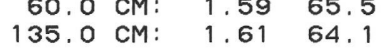

$0>[2.65 / 6.61 \mathrm{C} 0 / 1.5 / 4]$

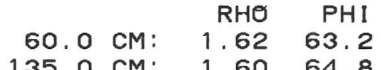
135.0 CM: 1.6064 .8

$0>[2.65 / 6.61 \mathrm{C} 0 / 1.5 / 4]$ 60.0 CM: $0.00 \quad 0.0$ $135.0 \mathrm{cM}: 1.57$ 66.6

$0>[2.65 / 6.61 \mathrm{C} 0 / 1.5 / 4]$ 60.0 CM: $\begin{array}{rrr}\mathrm{RHO} & \mathrm{PHI} \\ 1.60 & 64.5\end{array}$ $135.0 \mathrm{~cm}: 0.00 \quad 0.0$

$0>[2.65 / 6.61 \mathrm{C} 0 / 1.5 / 4]$ $60.0 \mathrm{~cm}: \quad \begin{array}{rr}\mathrm{RHO} & \mathrm{PHI} \\ 68.1 & 1.54\end{array}$ $135.0 \mathrm{CM}: 1.56 \quad 66.8$

o) $[2.65 / 6.61 \mathrm{C} 0 / 1.5 / 4]$

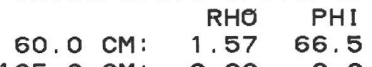

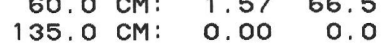

$0>[2.65 / 6.61 \mathrm{C} 0 / 1.5 / 4]$

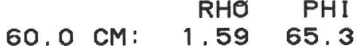
$135.0 \mathrm{cM}: 1.56$ 66.8

$0>[2.65 / 6.61 \mathrm{C} 0 / 1.5 / 4]$ $60.0 \mathrm{CM}: \quad \begin{array}{rr}\mathrm{RHO} \\ 1.54 & \mathrm{PHI}\end{array}$ $135.0 \mathrm{CM}: 1.59$ 65.3

$0>[2.65 / 6.61 \mathrm{C} 0 / 1.5 / 4]$ $60.0 \mathrm{CM}: \quad \begin{array}{r}\text { RHO } \\ 1.65\end{array}$ $135.0 \mathrm{CM}: 1.5567 .8$ 


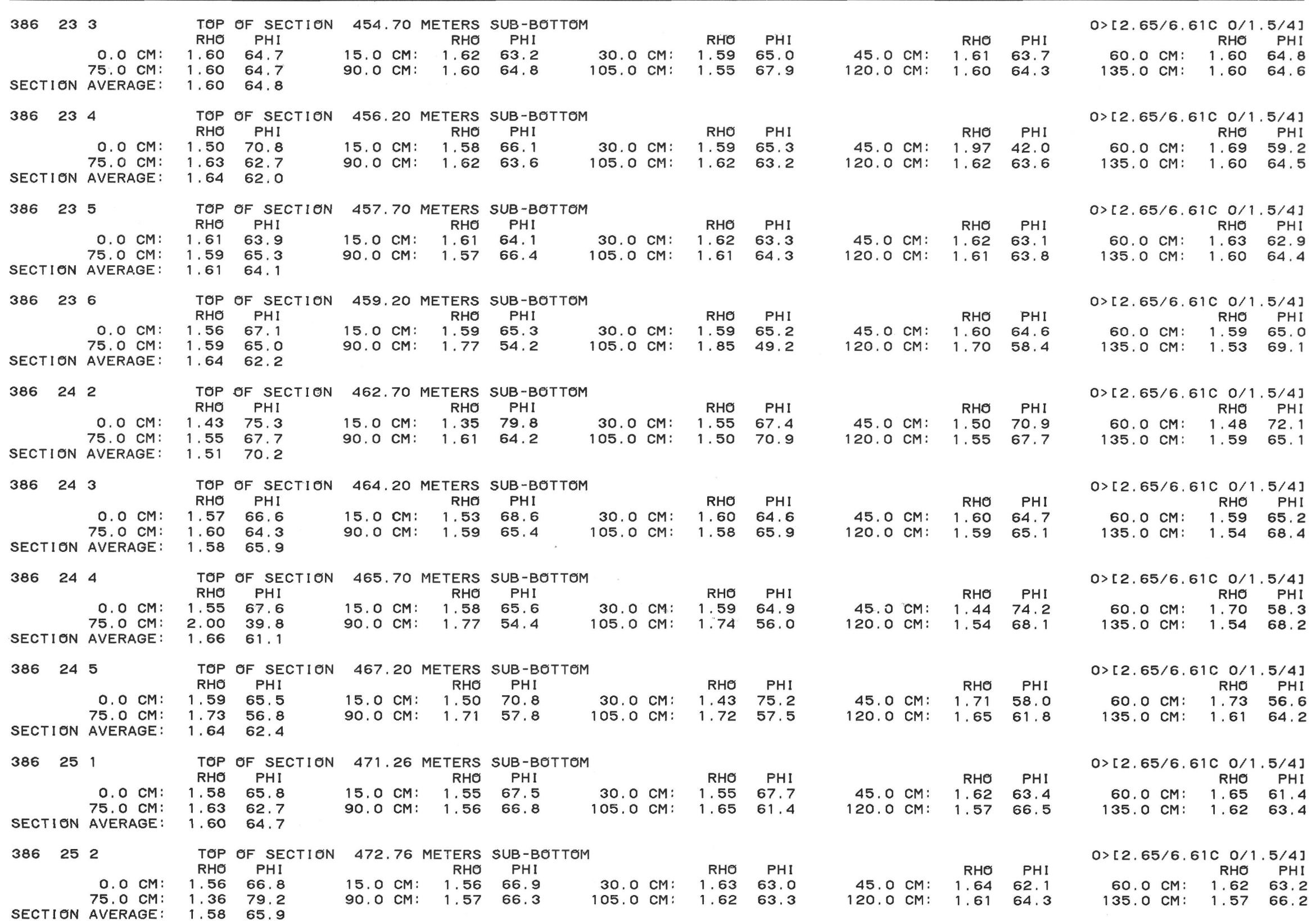


$\begin{array}{lll}386 & 25 & 3\end{array}$

TOP OF SECTION 474.26 METERS SUB-BOTTOM

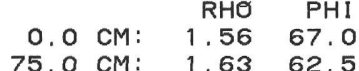

$\begin{array}{rrr}15.0 \mathrm{CM}: & \text { RHO } & \text { PHI } \\ 1.61 & 64.2\end{array}$

30.0 CM: $\begin{array}{rrr}\text { RHO } & \mathrm{PHI} \\ 1.65 & 61.4\end{array}$

$90.0 \mathrm{CM}: 1.6561 .8 \quad 105.0 \mathrm{CM}: 1.6362 .9$

SECTION AVERAGE: $1.63 \quad 62.6$

386254

TOP OF SECTION 475.76 METERS SUB-BOTTOM

$\begin{array}{rrr}0.0 \mathrm{CM}: & \text { RHO } & \mathrm{PHI} \\ 75.59 & 65.4\end{array}$

15. $\mathrm{cM}$ : RHO PHI

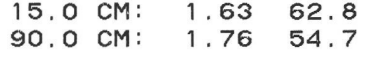

30. $\mathrm{CM}: \mathrm{RHO} \mathrm{PHI}$

$\begin{array}{rrr}30.0 \mathrm{CM}: & 1.63 & 63.0 \\ 105.0 \mathrm{CM}: & 1.74 & 55.9\end{array}$

$\begin{array}{ll}1.65 & 61.3 \\ 1.69 & 59.1\end{array}$

TOP OF SECTION 477.26 METERS SUB-BOTTOM

$386 \quad 25 \quad 5$

0.O CM: 1.76 RH.

15.0 CM: $\quad \begin{array}{rrr}\text { RH } & \text { PHI } \\ 1.57 & 66.5\end{array}$

30.0 CM: $\begin{array}{rr}\mathrm{RHO} & \mathrm{PHI} \\ 1.59 & 65.2\end{array}$

.5965 .2

90.0 CM: 1.5965 .3

105. $\mathrm{CM}: \quad 1.58$ 65.8

SECTION AVERAGE:

TOP OF SECTION 478.76 METERS SUB-BOTTOM

386256

$\mathrm{RHO} \mathrm{PHI}$

0.O CM: 1.51 70.3

15.0 CM: $\quad \begin{array}{rr}\text { RHO } & \text { PHI } \\ 70.50 & 70.5\end{array}$

30. $\mathrm{CM}: \quad \begin{array}{r}\mathrm{RHO} \\ \mathrm{PHI}\end{array}$

.68 59.4

$90.0 \mathrm{CM}: 1.68 \quad 59.9$

105.0 CM: 1.6760 .3

SECTI ON AVERAGE

TOP OF SECTION 481.80 METERS SUB-BOTTOM

386262

$0.0 \mathrm{CM}: \quad \mathrm{RHO} \mathrm{PHI} \quad \mathrm{RHO} \mathrm{PHI}$ RHO PHI

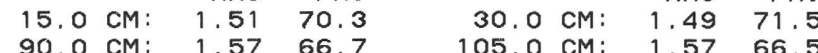

SECTION AVERAGE: 1.5468 .1

$386 \quad 263$

TOP OF SECTION 483.30 METERS SUB-BOTTOM

$\begin{array}{rrr}0.0 \text { CM: } & \text { RHO } & \text { PHI } \\ 1.54 & 68.5\end{array}$

15.0 1 RHO PHI

75. O CM: $\quad 1.55$ 68.0

SECTION AVERAGE: 1.5567 .6

$386 \quad 264$

TOP OF SECTION 484,80 METERS SUB-BOTTOM

$\begin{array}{rrr}0.0 \mathrm{CM}: & 1.57 & 66.4\end{array}$

$\begin{array}{rrr} & \text { RHO } & \text { PHI } \\ 15.0 \mathrm{CM}: & 1.55 & 67.5\end{array}$

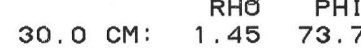

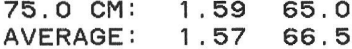

$386 \quad 26 \quad 5$

TOP OF SECTION 486.30 METERS SUB-BOTTOM

$\mathrm{RHO} \mathrm{PHI}$

0.0 CM: 1.6064 .9

15.0 CM: RHO PHI

$\begin{array}{lll}75.0 \mathrm{CM}: & 1.59 & 65.3 \\ \text { AVERAGE: } & 1.61 & 63.9\end{array}$

$90.0 \mathrm{CM}: 1.5965 .2$

RHO $\mathrm{PHI}$

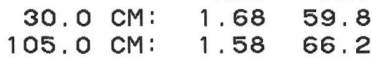

SECTION AVERAGE

TOP OF SECTION 491.30 METERS SUB-BOTTOM

$386 \quad 272$

$\begin{array}{rr}\text { RHO } & \text { PHI } \\ 1.58 & 65.7\end{array}$

15.0 CM: $\quad \begin{array}{r}\text { RHO } \\ 1.51 \text { PHI }\end{array}$

30.0 $\mathrm{CM}: \mathrm{RHO} \mathrm{PHI}$

$\begin{array}{rrr}0.0 \mathrm{CM}: & 1.58 & 65.7 \\ 75.0 \mathrm{CM}: & 1.59 & 65.2\end{array}$

90. $\mathrm{CM}: \quad 1.6064 .5$

$\begin{array}{rrr}105.0 \mathrm{CM}: & 1.56 & 66.8 \\ & 1.52 & 69.6\end{array}$

SECTION AVERAGE: 1.57 66.6

TOP OF SECTION 492.80 METERS SUB-BOTTOM

$386 \quad 27 \quad 3$

RHO PHI

O. 0 CM: $\quad 1.7158 .0$

$\begin{array}{rrr}15.0 \mathrm{CM}: & 1.64 & 62.0 \\ 90.0 \mathrm{CM}: & 1.56 & 66.8\end{array}$

$30 . \mathrm{CM}: \mathrm{RHO} \quad \mathrm{PHI}$

1.5269 .8

45.0 CM: $\begin{array}{rr}\mathrm{RHO} & \mathrm{PHI} \\ 1.76 & 54.8\end{array}$ $120.0 \mathrm{CM}: 1.64 \mathrm{62}$.

45. O CM: $\quad 1.6760$.

45. $\mathrm{CM}: \quad \begin{array}{r}\mathrm{RHO} \\ \mathrm{PHI}\end{array}$

SECTION AVERAGE
45.0 CM: $\begin{array}{rr}\text { RHO } & \mathrm{PHI} \\ 1.61 & 63.7\end{array}$

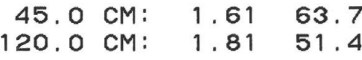

45. O CM: $\begin{array}{rrr}\text { RHO } & \text { PHI } \\ 1.61 & 64.3\end{array}$ 120.0 CM: 1.4672 .9

45. $\mathrm{CM}: \mathrm{RHO} \mathrm{PHI}$

RHO PHI

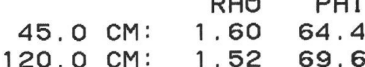

C.

45.0 cM: $\begin{array}{rr}\mathrm{RHO} & \mathrm{PH} \\ 1.53 & 68.8\end{array}$

$120.0 \mathrm{CM}: 1.6263 .6$

120.0 CM: 1.68 59.8

4HO PHI $\begin{array}{rrr}45.0 \mathrm{CM}: & 1.63 & 63.0 \\ 120.0 \mathrm{CM}: & 1.55 & 67.4\end{array}$

$0>[2.65 / 6.61 \mathrm{C} 0 / 1.5 / 4]$ $60.0 \mathrm{cM}: \quad \begin{array}{r}\mathrm{RHO} \\ 1.59\end{array}$ $135.0 \mathrm{cM}: 1.60$ 64.3

$0>[2.65 / 6.61 \mathrm{C} \quad 0 / 1.5 / 4]$ $60.0 \mathrm{cM}: \quad \begin{array}{rr}\mathrm{RHO} & \mathrm{PHI} \\ 1.67 & 60.0\end{array}$ 135.0 CM: 1.8251 .1

$0>[2.65 / 6.61 \mathrm{C} \quad 0 / 1.5 / 4]$ $60.0 \mathrm{CM}: \quad \begin{array}{r}\mathrm{RHO} \\ 1.60\end{array}$ $135.0 \mathrm{~cm}: \quad 1.1293 .9$

$0>[2.65 / 6.61 \mathrm{C} 0 / 1.5 / 4]$ 60.0 CM: 1.63 R3. $135.0 \mathrm{CM}: \quad 1.70 \quad 58.3$

$0>[2.65 / 6.61 \mathrm{C} \quad 0 / 1.5 / 4]$ 60.0 cM: $\quad$ RHO PHI $135.0 \mathrm{cM}: 1.57 \quad 66.4$

$0>[2.65 / 6.61 \mathrm{C} 0 / 1.5 / 4]$

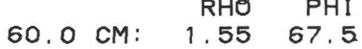
$135.0 \mathrm{CM}: 1.5567 .7$

$0>[2.65 / 6.61 \mathrm{C} 0 / 1.5 / 4]$ $60.0 \mathrm{CM}: \quad \begin{array}{r}\mathrm{RHO} \\ 1.61\end{array}$ $135.0 \mathrm{cM}: 1.6064 .7$

$0>[2.65 / 6.61 \mathrm{C} \quad 0 / 1.5 / 4]$ $60.0 \mathrm{CM}: \quad \begin{array}{r}\text { RHO } \\ 1.59\end{array}$ $\begin{array}{lll}135.0 \mathrm{CM}: & 1.57 & 66.7\end{array}$

$0>[2.65 / 6.61 \mathrm{C} 0 / 1.5 / 4]$ 45.0 cM: $\quad \begin{array}{rr}\text { RHO } & \text { PHI } \\ 1.60 & 64.9\end{array}$ $\begin{array}{rrr}45.0 \mathrm{CM}: & 1.60 & 64.9 \\ 120.0 \mathrm{CM}: & 1.60 & 64.6\end{array}$ $\begin{array}{rrr}60.0 \mathrm{cM}: & \text { RHO } & \mathrm{PHI} \\ 1.54 & 68.3\end{array}$ 135. 0 CM: 1.5766 .4

$0>[2.65 / 6.61 \mathrm{C} \mathrm{O/1.5/4]}$

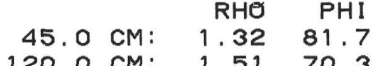

60.0 CM: 1.5468 .3 


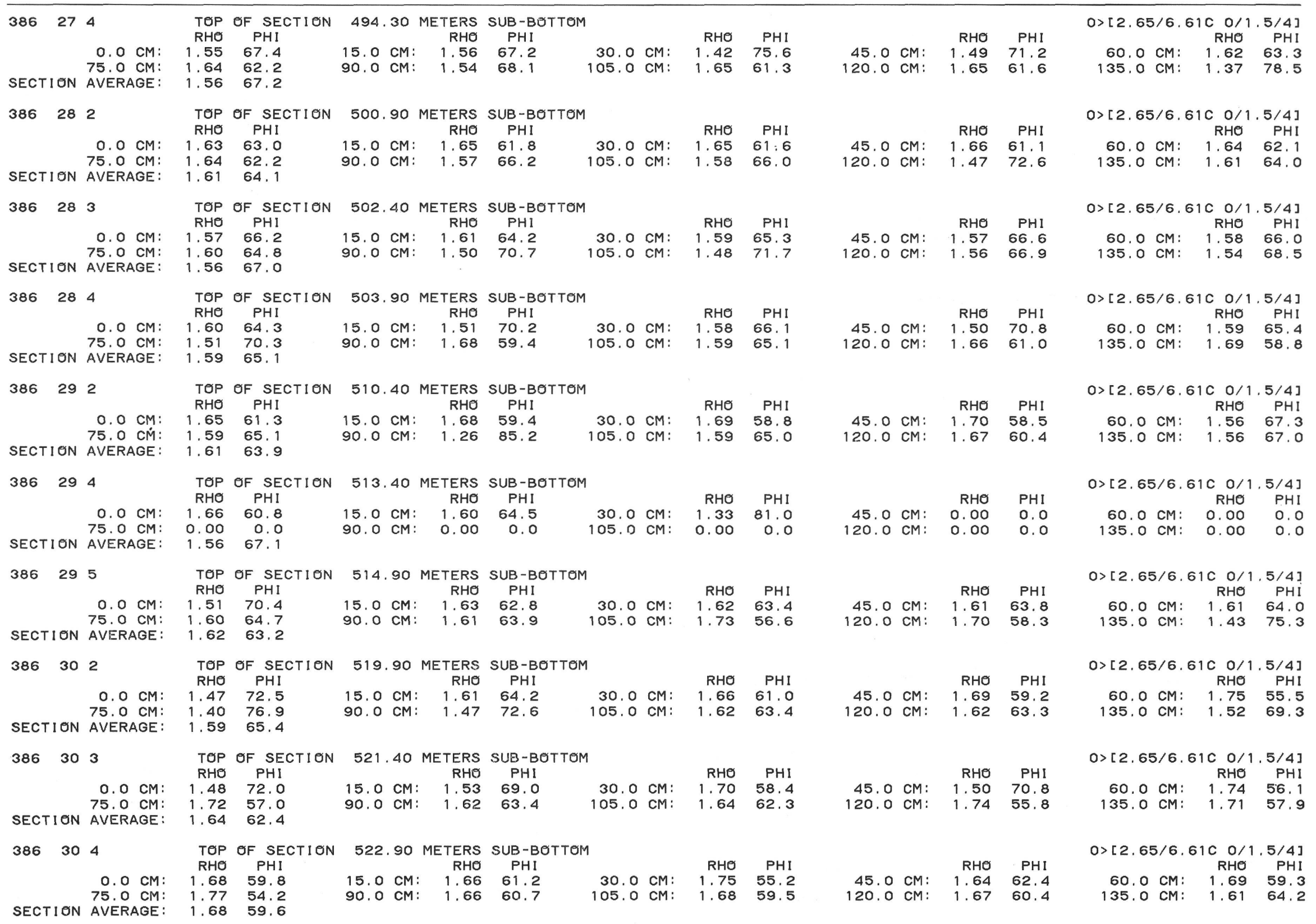


$38630 \quad 5$

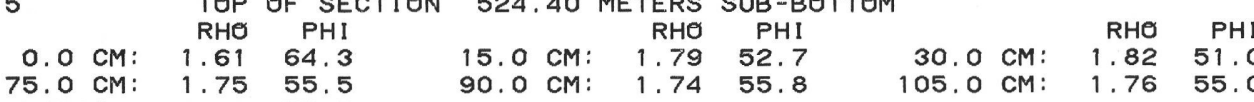
SECTION AVERAGE: $1.75 \quad 55.5$

386306

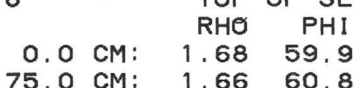
SECTION AVERAGE: 1.66 61.1

$\begin{array}{lll}386 & 31 & 1\end{array}$

\section{TOP OF SECTION $\mathbf{5 3 7 . 7 8}$ METERS SUB-BOTTOM}

RHO PHI

$0.0 \mathrm{CM}: 1.5070 .6$

SECTION AVERAGE: $\begin{array}{lll}7.65 & 61.4 \\ 1.63 & 62.9\end{array}$

RHO PHI

90.0 CM: $\quad 1.60$ 64.9

30. 0 CM:

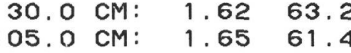

TOP OF SECTION 539.28 METERS SUB-BOTTOM

$386 \quad 312$

O.O CM:

RHO PHI

$\begin{array}{ll}.61 & 64.0 \\ .67 & 60.5\end{array}$

$15.0 \mathrm{CM}: \quad 1.65 \quad 61.3$

$30.0 \mathrm{CM}: \mathrm{RHO} \quad \mathrm{PHI}$

$\begin{array}{lll}75.0 \mathrm{CM}: & 1.67 & 60.5 \\ \text { AVERAGE: } & 1.66 & 61.0\end{array}$

$386 \quad 31 \quad 3$

\section{TOP OF SECTION 540.78 METERS SUB-BOTTOM}

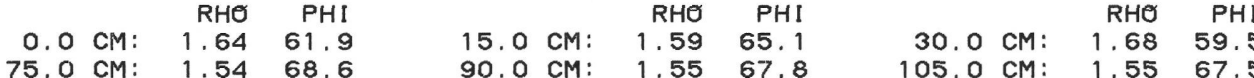

SECTION AVERAGE: 1.59 . 65.5

10

TOP OF SECTION 542,28 METERS SUB-BOTTOM

$386 \quad 314$

0. $\mathrm{CM}: \mathrm{RHO}$ PHI

75.0 CM: $\quad 1.50 \quad 71.0$

15.0 CM: 1.61 PHI

30. $\mathrm{CM}: \quad \mathrm{RHO} \mathrm{PHI}$

$1.64 \quad 62.4$

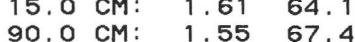

105.0 CM: 1.6064 .8

SECTION AVERAGE

TOP OF SECTION 543.78 METERS SUB-BOTTOM

$386 \quad 31 \quad 5$

0.0 CM: 1.5269 .3

15.0 CM: 1.5965

$30.0 \mathrm{CM}: 1.74$ PHI

$\begin{array}{lll}75.0 \mathrm{CM}: & 1.77 & 54.4 \\ \text { AVERAGE: } & 1.71 & 58.1\end{array}$

$386 \quad 31 \quad 6$

TOP OF SECTION 545.28 METERS SUB-BOTTOM

RHO PHI

0.0 CM: 1.6561 .8

$\begin{array}{lll}75.0 \mathrm{CM}: & 1.83 & 50.2 \\ \text { AVERAGE: } & 1.73 & 56.8\end{array}$

15. $\mathrm{CM}$ : RHO PHI

$\begin{array}{lll} & & \\ 90.0 \mathrm{CM}: & 1.67 & 60.6 \\ & 1.83 & 50.3\end{array}$

30. $\mathrm{CM}$ : $\mathrm{RHO} \mathrm{PHI}$

$\begin{array}{rrr}105.0 \mathrm{CM}: & 1.70 & 58.7 \\ & 1.74 & 56.2\end{array}$

SECTIION AVERAGE:

TOP OF SECTION 556.55 METERS SUB-BOTTOM

$38632 \quad 1$

0.0 CM: $\begin{array}{rr}\text { RHO } & \text { PHI } \\ 07.7 & 57.7\end{array}$

75.0 CM: 1.68 60.0

15.0 CM: $1.73 \quad 56.7$

30.0 CM: \begin{tabular}{rrr} 
RHO & PHI \\
\hline & 1.69 & 59.3
\end{tabular}

0.0 CM: 1.7455 .9

$45.0 \mathrm{CM}: \quad \mathrm{RHO} \quad \mathrm{PH}$

$386 \quad 32 \quad 2$

TOP OF SECTION 558.05 METERS SUB-BOTTOM

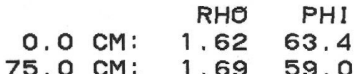
SECTION AVERAGE: 1.65 61.3 $\begin{array}{lllll}15.0 \mathrm{~cm}: & \begin{array}{r}\mathrm{RHO} \\ 1.66\end{array} 60.9 & \mathrm{PHI} & \mathrm{RHO} & \mathrm{PHI}\end{array}$

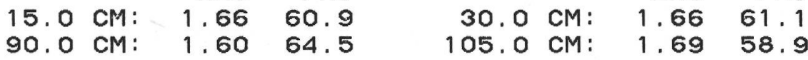

45. O cM: RHO PHI 120.0 CM: 1.78 53.8

RHO PHI $120.0 \mathrm{CM}: 1.6263$.

RHO PHI

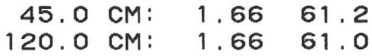
$\begin{array}{rrr}45.0 \mathrm{CM}: & 1.66 & 60.9 \\ 120.0 \mathrm{CM}: & 1.67 & 60.3\end{array}$

45.0 CM: $\begin{array}{rrr}\mathrm{RHO} & \mathrm{PHI} \\ 1.58 & 66.0\end{array}$ $\begin{array}{rrr}\text { 45.0 CM: } & 1.58 & 66.0 \\ 120.0 \mathrm{CM}: & 1.55 & 67.5\end{array}$

45. $\mathrm{CM}: \quad$ RHO $\mathrm{PHI}$ 45.0 CM: $1.54 \quad 68.2$

\section{RHO $\mathrm{PHI}$} $\begin{array}{rrr}45.0 \mathrm{CM}: & 1.80 & 52.5 \\ 120.0 \mathrm{CM}: & 1.66 & 60.7\end{array}$

45. 0 CM: $\quad \begin{array}{rlr}\text { RHO } & \text { PHI } \\ 1.71 & 57.9\end{array}$ $120.0 \mathrm{CM}: 1.7456 .1$

45. O CM: $\begin{array}{rr}\text { RHO } & \text { PHI } \\ 1.71 & 57.6\end{array}$ $120.0 \mathrm{CM}: \quad 1.75 \quad 55.1$

$0>[2.65 / 6.61 \mathrm{C} 0 / 1.5 / 4]$ 60.0 cM: $\quad \begin{array}{rr}\mathrm{RHO} & \mathrm{PHI}\end{array}$ 135.0 CM: $1.77 \quad 54.2$

$0>[2.65 / 6.61 \mathrm{C} 0 / 1.5 / 4]$ 60.0 CM: $1.74 \quad 55.9$ 135.0 CM: $1.45 \quad 73.7$

$0>[2.65 / 6.61 \mathrm{C} \quad 0 / 1.5 / 4]$ 60.0 CM: 1.65 ( $135.0 \mathrm{cM}: \quad 1.64$ 62.2

$0>[2.65 / 6.61 \mathrm{C} \quad 0 / 1.5 / 4]$ $60.0 \mathrm{cM}: \quad \begin{array}{rr}\mathrm{RHO} & \mathrm{PHI} \\ 1.66 & 60.8\end{array}$ $135.0 \mathrm{cM}: 1.6760 .6$

$0>[2.65 / 6.61 \mathrm{C} 0 / 1.5 / 4]$ $60.0 \mathrm{~cm}: \quad \begin{array}{rr}\mathrm{RHO} & \mathrm{PHI} \\ 1.58 & 66.1\end{array}$ $135.0 \mathrm{CM}: 0.00$ 0.

$0>[2.65 / 6.61 \mathrm{C} 0 / 1,5 / 4]$

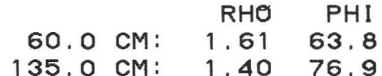

$0>[2.65 / 6.61 \mathrm{C} 0 / 1.5 / 4]$ $60.0 \mathrm{CM}: \quad \begin{array}{r}\text { RHO } \\ 1.79\end{array}$ $135.0 \mathrm{~cm}: 1.64$ 62.0

$0>[2.65 / 6.61 \mathrm{C} \quad 0 / 1.5 / 4]$ $60.0 \mathrm{cM}: \quad 1.71 \quad 57.9$ 135.0 CM: 1.6959 .0

$0>[2.65 / 6.61 \mathrm{C} \quad 0 / 1.5 / 4]$

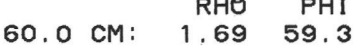
$135.0 \mathrm{CM}: 1.6263 .5$ $0>[2.65 / 6.61 \mathrm{C} \quad 0 / 1.5 / 4]$ $\begin{array}{rrrrrr} & \text { RHO } & \text { PHI } & & \text { RHO } & \text { PHI } \\ 15.0 \mathrm{CM}: & 1.68 & 59.6 & 60.0 \mathrm{cM}: & 1.67 & 60.1 \\ 120.0 \mathrm{CM}: & 1.64 & 61.9 & 135.0 \mathrm{cM}: & 1.61 & 64.1\end{array}$

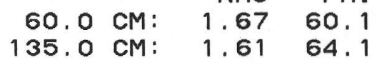




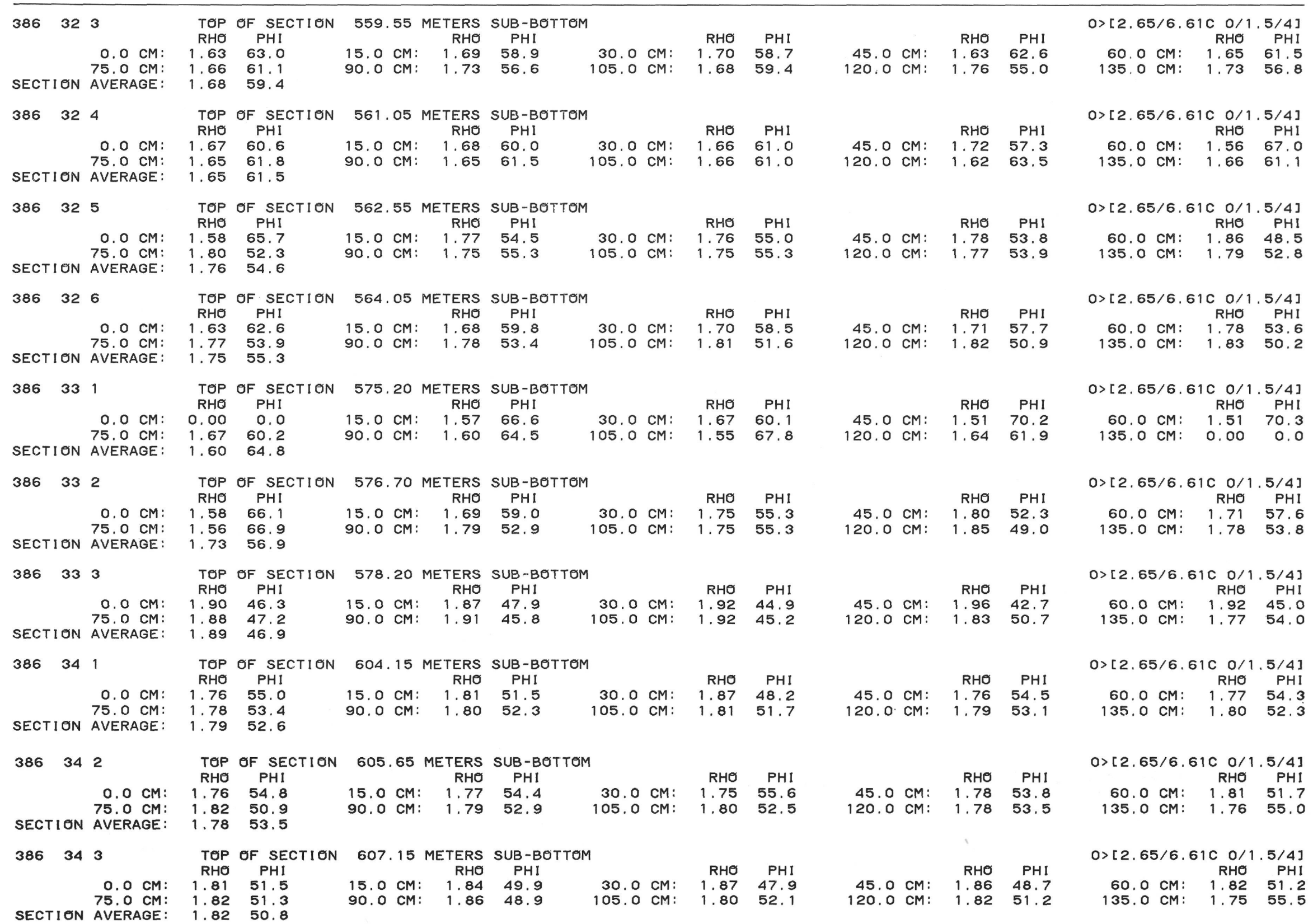


TOP OF SECTION 608.65 METERS SUB-BOTTOM

RHO PHI

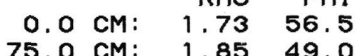
$\begin{array}{rll}75.0 \text { CM: } & 1.85 & 49.0 \\ \text { SECTION AVERAGE: } & 1.82 & 50.8\end{array}$

$\begin{array}{rrr} & \text { RHO } & \text { PHI } \\ 15.0 \mathrm{CM} & 1.77 & 54.5\end{array}$

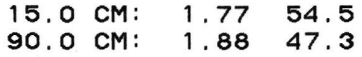

$30.0 \mathrm{CM}: \quad \begin{array}{rr}\mathrm{RHO} & \mathrm{PHI} \\ 1.80 & 52.1\end{array}$

$105.0 \mathrm{CM}: \quad 1.93 \mathrm{44}$.

TOP OF SECTION 610.15 METERS SUB-BOTTOM

386345

\section{RHO $\mathrm{PHI}$ \\ $15.0 \mathrm{CM}$ RHO PHI}

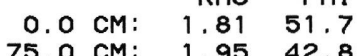

90.0 CM: $\quad 1.86$ 48.5

30.0 CM: $\quad \begin{array}{rr}\mathrm{RHO} & \mathrm{PHI}\end{array}$

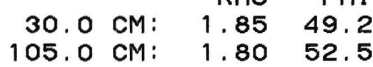
SECTION AVERAGE: $1.86 \quad 48.6$

386346

\section{TOP OF SECTION 611.65 METERS SUB-BOTTOM}

$0.0 \mathrm{CM}:$
$75.0 \mathrm{CM}:$

15. $\mathrm{CM}: \quad \mathrm{RHO} \quad \mathrm{PHI}$

20.0 $\mathrm{PHI}$

$1.82 \quad 51.0$

15.0 CM: $1.83 \quad 50.6$

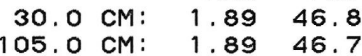

SECTION AVERAGE: 1.8946 .7

$386 \quad 352$

TOP OF SECTION 633.50 METERS SUB-BOTTOM

RHO PHI

$\begin{array}{lll}75.0 \mathrm{CM}: & 1.92 & 44.9 \\ \text { AVERAGE: } & 1.92 & 44.6\end{array}$

$15.0 \mathrm{cM}: \quad 1.94 \quad 43.7$

30.0 CM: $\quad \begin{array}{rrr}\text { RHO } & \text { PHI } \\ 1.94 & 43.6\end{array}$

90.0 CM: 1.9145 .5

386353

TOP OF SECTION 635.00 METERS SUB-BOTTOM

RHO PHI

$\begin{array}{lll}0.0 \mathrm{CM}: & 1.88 & 47.3 \\ 75.0 \mathrm{CM}: & 1.93 & 44.5\end{array}$

RHO PHI

$1.94 \quad 43.7$

30. $\mathrm{CM}: \quad \begin{array}{r}\mathrm{RHO} \\ \mathrm{PHI}\end{array}$

SECTION AVERAGE: $1.93 \quad 44.4$

$90.0 \mathrm{CM}: 1.93 \quad 44.5 \quad 105.0 \mathrm{cM}: 1.96 \quad 42.7$

$386 \quad 354$

TOP OF SECTION 636.50 METERS SUB-BOTTOM

RHO PHI RH: PHI RHO PHI

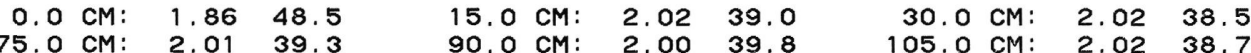

SECTION AVERAGE: $1.97 \quad 41.6$

$386 \quad 35 \quad 5$

TOP OF SECTION 638.00 METERS SUB-BOTTOM

$\mathrm{RHO} \mathrm{PHI}$

O. $0 \mathrm{CM}: \quad 1.83 \quad 50.4$

SECTION AVERAGE: $1.93 \quad 44.1$

$\begin{array}{rrr}15.0 \mathrm{~cm}: & \text { RHO } & \text { PHI } \\ 1.94 & 43.7\end{array}$

30. $\mathrm{OM}: \mathrm{RHO} \mathrm{PHI}$

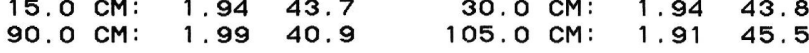

TOP OF SECTION 639.50 METERS SUB-BOTTOM

386356

O. $0 \mathrm{CM}:$

$\begin{array}{rrr} & \text { RHO } & \text { PHI } \\ 15.0 \mathrm{CM}: & 1.94 & 43.8\end{array}$

RHO PHI

1.9046 .1

90. CM: 1.9344 .3

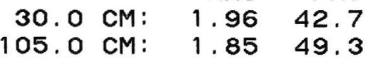

SECTION AVERAGE: 1.9145 .7

$\begin{array}{lll}386 & 36 & 1\end{array}$

TOP OF SECTION 641.50 METERS SUB-BOTTOM

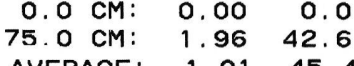

$\begin{array}{lll}15.0 \mathrm{CM}: & \mathrm{RHO} & \mathrm{PHI} \\ & 0.00 & 0.0\end{array}$

30.0 CM: $\begin{array}{rr}\text { RHO } & \text { PHI } \\ 1.22 & 87.7\end{array}$

$90.0 \mathrm{CM}: 1.96 \quad 42.3$

105.0 CM: $1.94 \quad 43.9$

SECTI ON AVERAGE:

TOP OF SECTION 643.00 METERS SUB-BOTTOM

386362

RHO PHI

$\begin{array}{rrr}0.0 \mathrm{CM}: & 1.81 & 51.8 \\ 75.0 \mathrm{CM}: & 1.96 & 42.5\end{array}$

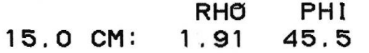

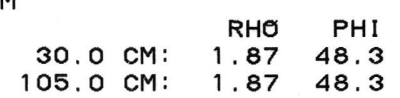

45. 0 CM: $\quad \begin{array}{rr}\text { RHO } & \text { PHI } \\ 1.79 & 53.1\end{array}$ 120.0 CM: 1.94 44.0

45. $\mathrm{CM}: \mathrm{RHO} \quad \mathrm{PHI}$ $\begin{array}{rrr}45.0 \mathrm{CM}: & 1.90 & 46.0 \\ 120.0 \mathrm{CM}: & 1.80 & 52.4\end{array}$

$\mathrm{RHO} \quad \mathrm{PHI}$

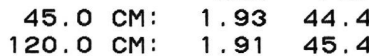

45. $0 \mathrm{cM}: \quad$ RHO PHI $\begin{array}{rrr}45.0 \mathrm{CM}: & 1.96 & 42.6 \\ 120.0 \mathrm{CM}: & 1.92 & 45.0\end{array}$

45. 0 CM: $\quad \begin{array}{rr}\text { RHO } & \text { PHI } \\ 1.94 & 43.8\end{array}$ 120.0 CM: 1.9344 .0

45. O cM: 2 RHO PHI $120.0 \mathrm{CM}: \quad 1.94$ 43.8

45. C CM: $\quad$ RHO PHI $\begin{array}{rrr}45.0 \mathrm{CM}: & 1.93 & 44.0 \\ 120.0 \mathrm{CM}: & 1.96 & 42.5\end{array}$

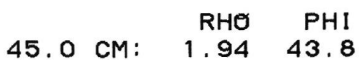
$\begin{array}{rrr}45.0 \mathrm{CM}: & 1.94 & 43.8 \\ 120.0 \mathrm{CM}: & 1.86 & 48.7\end{array}$

45.0 CM: $\begin{array}{rr}\text { RHO } & \text { PHI } \\ 1.82 & 51.2\end{array}$ $\begin{array}{rrr}45.0 \mathrm{CM}: & 1.82 & 51.2 \\ 120.0 \mathrm{CM}: & 1.98 & 41.4\end{array}$

RHO PHI

45. $\mathrm{CM}: \quad 1.80 \quad 52.5$
$0>[2.65 / 6.61 \mathrm{C} 0 / 1.5 / 4]$ $60.0 \mathrm{cM}: \quad \begin{array}{r}\mathrm{RHO} \\ \mathrm{PHI}\end{array}$ $\begin{array}{rrr}135.0 \mathrm{CM}: & 1.77 & 53.9 \\ & & \end{array}$

$0>[2.65 / 6.61 \mathrm{C} 0 / 1.5 / 4]$

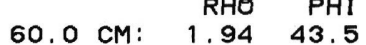
$135.0 \mathrm{CM}: 1.76 \quad 54.5$

$0>[2.65 / 6.61 \mathrm{C} 0 / 1.5 / 4]$ $\begin{array}{rrr}60.0 \mathrm{CM}: & \mathrm{RHO} & \mathrm{PHI} \\ & 1.91 & 45.8\end{array}$

$0>[2.65 / 6.61 \mathrm{C} 0 / 1.5 / 4]$

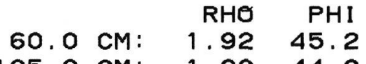
$135.0 \mathrm{CM}: 1.93 \quad 44.3$

$0>[2,65 / 6,61 \mathrm{C} 0 / 1.5 / 4]$

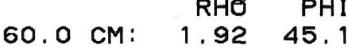
$135.0 \mathrm{CM}: 1.91 \quad 45.7$

$0>[2.65 / 6.61 \mathrm{C} 0 / 1.5 / 4]$ $60.0 \mathrm{CM}: \quad 2.00 \quad 39.9$ $135.0 \mathrm{CM}: 1.86 \quad 48.7$

$0>[2.65 / 6.61 \mathrm{C} 0 / 1.5 / 4]$ $60.0 \mathrm{cM}: 1.95 \quad 42.8$ 135.0 CM: $1.94 \quad 43.5$

$0>[2.65 / 6.61 \mathrm{C} 0 / 1.5 / 4]$ $60.0 \mathrm{~cm}: \begin{array}{rr}\mathrm{RHO} & \mathrm{PHI} \\ 1.94 & 43.4\end{array}$ 135.0 CM: 1.8847 .4

$0>[2.65 / 6.61 \mathrm{C} \quad 0 / 1.5 / 4]$ $60.0 \mathrm{cM}: \quad 1.91 \quad 45.6$ : $1.95-43,3$ $0>[2.65 / 6.61 \mathrm{C} 0 / 1.5 / 4]$ $60.0 \mathrm{cM}: \begin{array}{rrr}\text { RHO } & \text { PHI } \\ 1.86 & 48.6\end{array}$ 135.0 CM: $1.87 \quad 48.1$ 


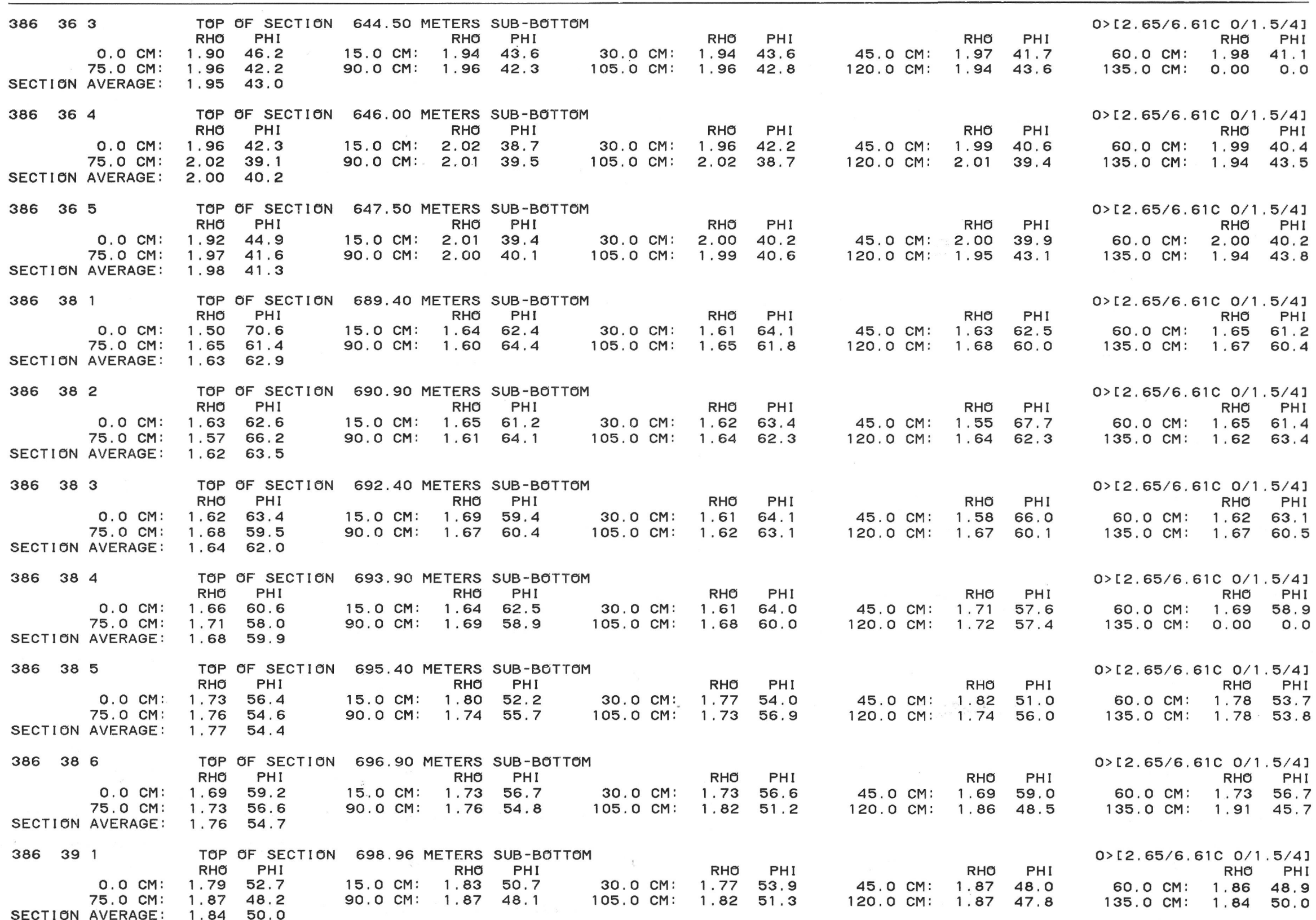




\section{$0.0 \mathrm{CM}:$
$75.0 \mathrm{CM}:$ \\ SECTI ON AVERAGE:}

RHO PH

$\begin{array}{ll}1.79 & 53.0 \\ 1.89 & 46.6\end{array}$

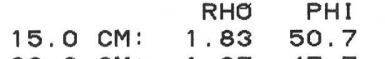

\begin{tabular}{rrr}
$30.0 \mathrm{CM}:$ & $\mathrm{RHO}$ & $\mathrm{PHI}$ \\
$105.0 \mathrm{CM}:$ & 1.83 & 50.5 \\
\hline & 1.84 & 49.6
\end{tabular}

$1.84 \quad 49.7$

90.0 CM: $1.87 \quad 47.7$

TOP OF SECTION 701.96 METERS SUB-BOTTOM

$\begin{array}{lll}386 & 39 & 3\end{array}$

O. 0 CM:

$1.74 \quad 56.0$

$\begin{array}{ll}1.85 & 49.4 \\ 1.84 & 49.7\end{array}$

15.0 CM: 1.86 PHI

90.0 CM: 1.84 50.1

RHO PHI

TOP OF SECTION 703.46 METERS SUB-BOTTOM

$\begin{array}{lll}386 & 39 & 4\end{array}$

O. $0 \mathrm{CM}:$

RHO
1.70
PHI

$\begin{array}{lll}75.0 \mathrm{CM}: & 1.57 & 66.6 \\ \text { AVERAGE: } & 1.70 & 58.4\end{array}$

15.0 CM: $\quad 1.77$ PHI

90.0 CM: $1.77 \quad 54.4$

30.0 CM: $\begin{array}{rr}\text { RHO } & \text { PHI } \\ 105 & 57.5\end{array}$

704.96 METERS SUB-BOTTOM

TOP OF SECTION 704.96 METERS SUB-BOTTOM
RHO PHI

$\begin{array}{lll}386 & 39 \quad 5\end{array}$

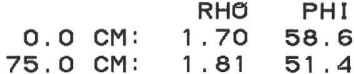

30. $\mathrm{CM}: \quad \begin{array}{rr}\text { RHO } & \mathrm{PH} \\ 1.75 & 55.4\end{array}$

1.7953 .2

SECTION AVERAGE:

TOP OF SECTION 706.46 METERS SUB-BOTTOM

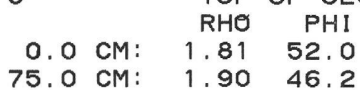

15.0 $\mathrm{CM}$ RHO PHI

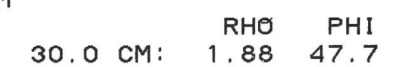
$90.0 \mathrm{CM}: \quad 1.89$ 46.9

105.0 CM: 1.89 46.9

SECTION AVERAGE: 1.8450 .1

$386 \quad 40 \quad 1$

TOP OF SECTION 708.00 METERS SUB-BOTTOM

$\mathrm{RHO} \mathrm{PHI}$

0.0 CM: $\quad 1.70 \quad 58.8$

$\begin{array}{lll}75.0 \text { CM: } & 1.76 & 54.8 \\ \text { SECTION AVERAGE: } & 1.74 & 56.2\end{array}$

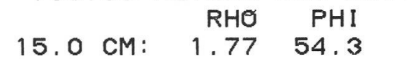

30.0 $\mathrm{RHO}$ PHI 90.0 CM: $1.76 \quad 54.8$ $\begin{array}{rrr}30.0 \mathrm{CM}: & 1.66 & 60.8 \\ 105.0 \mathrm{CM}: & 1.78 & 53.7\end{array}$

TOP OF SECTION 709.50 METERS SUB-BOTTOM

$386 \quad 402$ RHO PHI \begin{tabular}{rrr}
$0.0 \mathrm{CM}:$ & RHO & PHI \\
$75.0 \mathrm{CM}:$ & 1.74 & 55.9 \\
\hline & 1.74 .4
\end{tabular} $\begin{array}{rrr}15.0 \mathrm{CM}: & \begin{array}{r}\mathrm{RHO} \\ \mathrm{9}\end{array} \mathbf{\mathrm { PHI }} \\ \mathbf{5 8} 8.4\end{array}$ $30.0 \mathrm{CM} \quad \mathrm{RHO} \mathrm{PHI}$ 90.0 $\mathrm{CM}: \quad 1.7157 .7$ $105.0 \mathrm{CM}: \quad 1.68 \quad 59.5$ SECTION AVERAGE:

TOP OF SECTION 711.00 METERS SUB-BOTTOM

$386 \quad 403$

O CM: RHO PHI RHO PHI RHO PHI

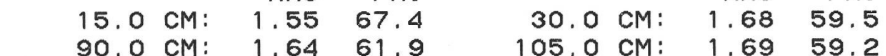

SECTION AVERAGE: 1.7157 .9

$\begin{array}{lll}386 & 41 & 1\end{array}$

PHI RHO PHI RHO PHI

$\begin{array}{rrr}0.0 & & \\ 75 & 0.00 & 0.0\end{array}$

SECTION AVERAGE: $1.76 \quad 54$.

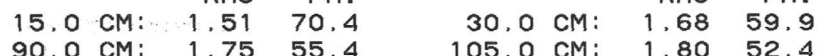

TOP OF SECTION 719.00 METERS SUB-BOTTOM

$386 \quad 412$

$0.0 \mathrm{CM}: \quad 1.83 \quad 50.7$

$75.0 \mathrm{CM}: \quad 1.89 \quad 46.5$

SECTION AVERAGE: $1.86 \quad 48.4$

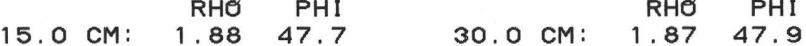
90.0 CM: $1.87 \quad 48.1$
45. O CM: $\quad \begin{array}{rrr}\text { RHO } & \text { PHI } \\ 1.82 & 51.1\end{array}$

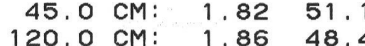

45. $\mathrm{RHO}$ PHI

45.0 CM: $\quad 1.83 \quad 50.7$

45. O CM: $\quad \begin{array}{rr}\text { RHO } & \text { PHI } \\ 55.75 & 57.8\end{array}$

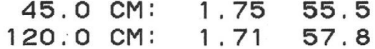

$45.0 \mathrm{CM}: \quad \mathrm{RHO} \quad \mathrm{PHI}$

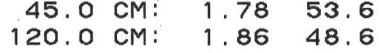

45. O CM: $\begin{array}{rr}\text { RHO } & \text { PHI } \\ 1.88 & 47.3\end{array}$ 120.0 CM: 1.83 50.6

45.0 CM: $\begin{array}{rr}\text { RHO } & \text { PHI } \\ 1.76 & 54.7\end{array}$

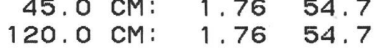

45.0 CM: $\quad \begin{array}{rr}\text { RHO } & \text { PHI } \\ 1.75 & 55.4\end{array}$ $120.0 \mathrm{cM}: 1.6959 .2$

45. O CM: $\quad \begin{array}{rr}\text { RHO } & \text { PHI }\end{array}$ $120.0 \mathrm{CM}: \quad 1.78 \quad 53.4$

45. O CM: $\quad \begin{array}{r}\text { RHO PHI } \\ \text { P. }\end{array}$

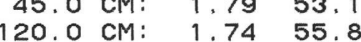

45. $\mathrm{CM}: \quad \mathrm{RHO} \quad \mathrm{PHI}$ $\begin{array}{rlll}45.0 & \text { CM: } & 1.87 & 48.3 \\ 120.0 & \text { CM: } & 1.85 & 49.1\end{array}$
$0>[2.65 / 6.61 \mathrm{C} 0 / 1.5 / 4]$ $60.0 \mathrm{CM}: 1.94 \quad 44.0$ $135.0 \mathrm{CM}: 1.7257 .5$

$0>[2.65 / 6.61 \mathrm{C} 0 / 1.5 / 4]$ $60.0 \mathrm{cM}: \quad \mathrm{RHO} \quad \mathrm{PHI}$ 135. O CM: 1.8548 .9

$0>[2.65 / 6.61 \mathrm{C} \quad 0 / 1.5 / 4]$ $60.0 \mathrm{cM}: \quad \begin{array}{rr}\mathrm{RHO} & \mathrm{PHI} \\ 1.56 & 67.1\end{array}$ $135.0 \mathrm{CM}: 1.76 \quad 54.7$

$0>[2,65 / 6.61 \mathrm{C} \quad 0 / 1.5 / 4]$ $60.0 \mathrm{CM}: \quad 1.8350 .6$ 135.0 CM: 1.80 52.0

$0>[2.65 / 6.61 \mathrm{C} 0 / 1.5 / 4]$

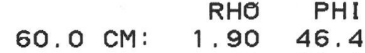
135.0 CM: $1.48 \quad 71.9$

$0>[2.65 / 6.61 \mathrm{C} \quad 0 / 1.5 / 4]$

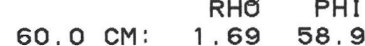
$135.0 \mathrm{cM}: 1.62$ 63.3

$0>[2.65 / 6.61 \mathrm{C} \quad 0 / 1.5 / 4]$ $\begin{array}{rrr}60.0 \mathrm{CM}: & \begin{array}{r}\text { RHO } \\ \text { PHI }\end{array} \\ 1353.0\end{array}$

$0>[2.65 / 6.61 \mathrm{C} 0 / 1.5 / 4]$ $60.0 \mathrm{cM}: \quad \begin{array}{r}\mathrm{RHO} \\ \mathrm{PHI}\end{array}$ $135.0 \mathrm{CM}: 1.90 \quad 45.9$

$0>[2.65 / 6.61 \mathrm{C} 0 / 1.5 / 4]$ $60.0 \mathrm{cM}: \quad \mathrm{RHO} \mathrm{PHI}$ 135. O CM: 1.60 64.6

$0>[2.65 / 6.61 \mathrm{C} 0 / 1.5 / 4]$ $60 . \mathrm{cM}: \quad \mathrm{RHO} \mathrm{PHI}$ $135.0 \mathrm{CM}: \quad 1.81 \quad 51.6$ 


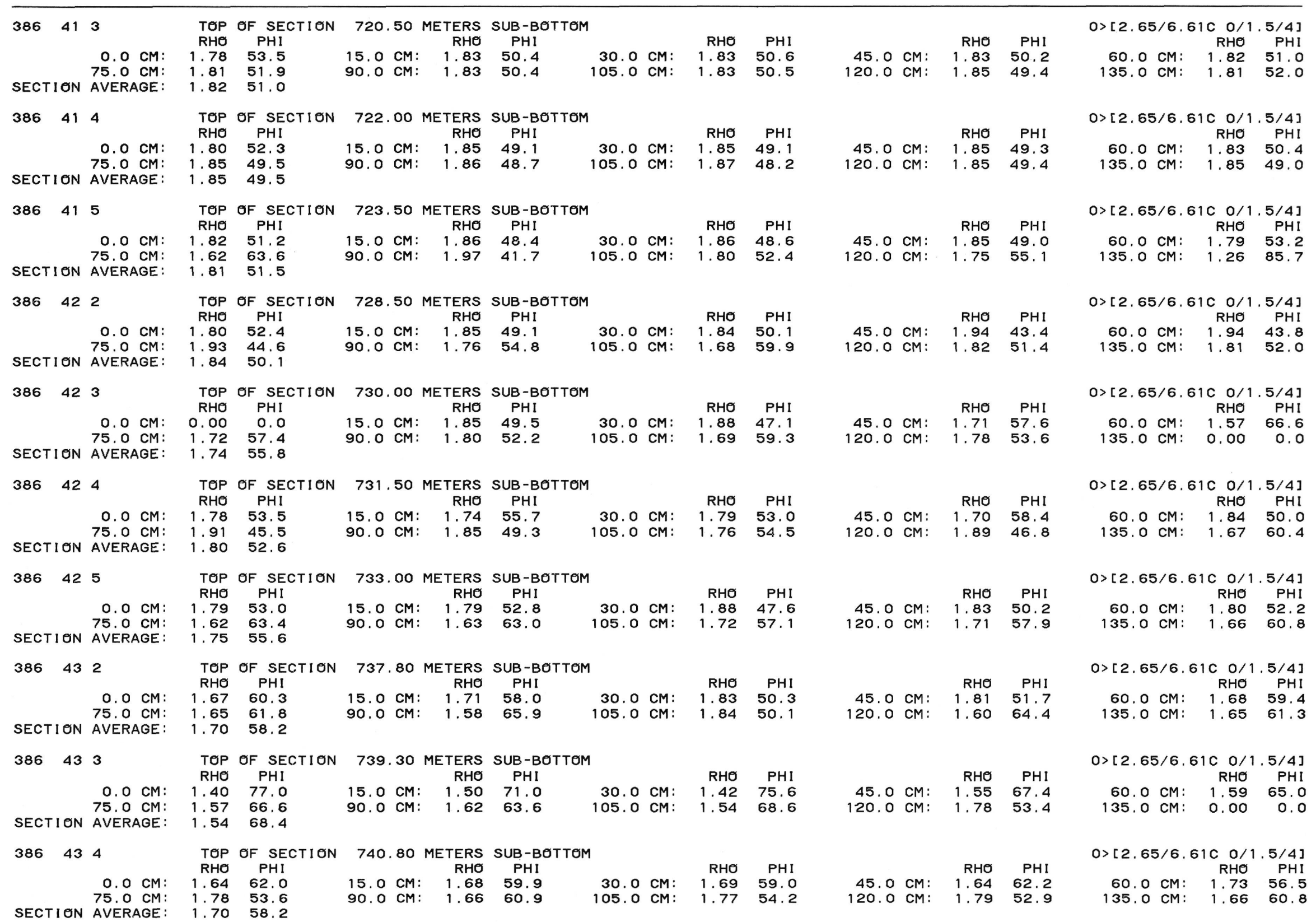


TOP OF SECTION 745.80 METERS SUB-BOTTOM

75.0 CM: $1.87 \quad 56.3$

15.0 CM: RHO PHI

90.0 CM: $1.74 \quad 55.9$

30.0 cM: $\quad$ RHO PHI

$105.0 \mathrm{CM}: \quad 1.7952 .8$

SECTION AVERAGE: $1.68 \quad 61.5$

386442

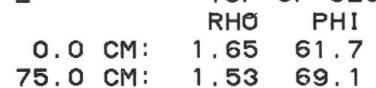

747.30 METERS SUB-BOTTOM

SECTION AVERAGE: $1.72 \quad 57.3$

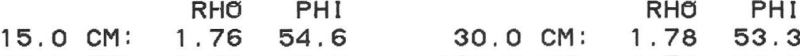

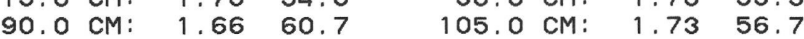

$386 \quad 443$

TOP OF SECTION 748.80 METERS SUB-BOTTOM

RHO PHI

0.0 CM: $\quad 1.69 \quad 59.2$

SECTION AVERAGE: 1.7953 .0

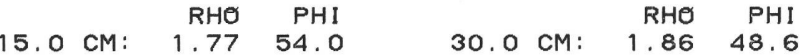

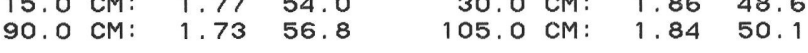

$386 \quad 44 \quad 4$

TOP OF SECTION 750.30 METERS SUB-BOTTOM

$\begin{array}{rrr}0.0 \mathrm{CM}: & 1.77 & \mathrm{RH} \\ 75.4 & \end{array}$

SECTION AVERAGE: $1.78 \quad 53.5$

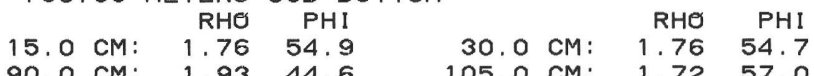

$\begin{array}{lll}386 & 45 & 1\end{array}$

TOP OF SECTION 765.40 METERS SUB-BOTTOM

$\mathrm{RHO} \quad \mathrm{PHI}$

$\begin{array}{rrr}0.0 \mathrm{CM}: & 1.64 & 62.4 \\ 75.0 \mathrm{CM}: & 1.79 & 52.8\end{array}$

15.0 cM: RHO PHI 1.67 RHO PHI

75. CM: $\quad 1.7952 .8$

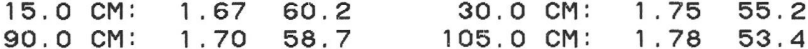

SECTI ON AVERAGE:

TOP OF SECTION 766.90 METERS SUB-BOTTOM

$386 \quad 45 \quad 2$

$0.0 \mathrm{CM}: \quad 1.62 \quad 63.7$

75. 0 CM: 1.60 64.9

15.0 $\mathrm{CM} \quad \mathrm{RHO} \mathrm{PHI} \quad \mathrm{RHO} \mathrm{PHI}$

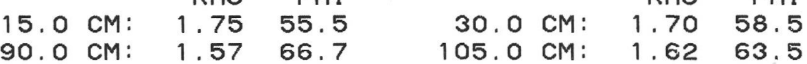

SECTION AVERAGE: 1.6561 .3

$386 \quad 45 \quad 3$

TOP OF SECTION 768.40 METERS SUB-BOTTOM

$0.0 \mathrm{CM}$ RHO PHI RHO PHI RHO PHI

$\begin{array}{lll}0.0 & 1.67 & 60.3\end{array}$

SECTION AVERAGE: $1.74 \quad 56.2$

$90.0 \mathrm{CM}: \quad 1.79$ 53.0 $105.0 \mathrm{CM}: 1.6959 .3$

$386 \quad 45 \quad 4$

TOP OF SECTION 769.90 METERS SUB-BOTTOM

$\begin{array}{rr}\mathrm{RHO} & \mathrm{PHI} \\ 1.76 & 55.0\end{array}$

O.O CM: $\quad 1.76 \quad 55.0$

SECTION AVERAGE: $\begin{array}{lll}1.80 & 52.3\end{array}$

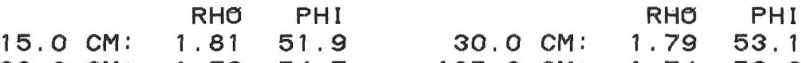

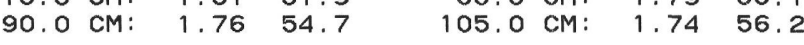

$\begin{array}{lll}386 & 45 \quad 5\end{array}$

TOP OF SECTION 771.40 METERS SUB-BOTTOM

$\mathrm{RHO}$
1.77

$0.0 \mathrm{CM}: \quad 1.77 \quad 53.9$

RHO PHI

$\begin{array}{lll}75.0 \mathrm{CM}: & 1.92 & 45.2 \\ & 1.91 & 45.5\end{array}$

90.0 CM: $1.94 \quad 43.8$

30. 0 CM: RHO PHI

305.

TOP OF SECTION 772.90 METERS SUB-BOTTOM

$386 \quad 456$

\begin{tabular}{rr}
$\mathrm{RHO}$ & $\mathrm{PHI}$ \\
.83 & 50.6 \\
\hline .96 & 4.96
\end{tabular}

0.0 CM: $\quad 1.83 \quad 50.6$

SECTION AVERAGE: $1.94 \quad 43.7$

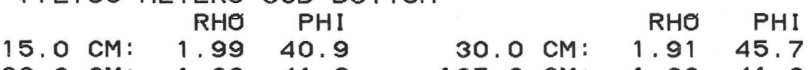
90.0 CM: 1.98 41.2 $105.0 \mathrm{cM}: 1.9841 .0$
$45.0 \mathrm{cM}: \quad \begin{array}{r}\mathrm{RHO} \\ \mathrm{PHI}\end{array}$ 120.0 CM: 1.6064 .8

45. O CM: $\quad$\begin{tabular}{rr} 
RHO & PHI \\
\hline &
\end{tabular} $120.0 \mathrm{cM}: 1.80 \quad 52.5$

O CM: RHO PHI 120.0 CM: 1.75 55.5

45. O CM: $\quad \begin{array}{rr}\text { RHO } & \text { PHI } \\ 1.76 & 54.8\end{array}$ $120.0 \mathrm{CM}: 1.7158 .0$

45. O CM: $\quad$ RHO PHI $45.0 \mathrm{CM}: \quad 1.63 \quad 63.0$

RHO PHI 45.0 CM: $1.46 \quad 73.3$

45. $\mathrm{CM}: \quad \mathrm{RHO} \quad \mathrm{PHI}$ $\begin{array}{lll}120.0 \mathrm{CM}: & 1.79 & 52.0 \\ & & \end{array}$

45. 0 CM: $\quad \begin{array}{rr}\text { RHO } & \mathrm{PHI}\end{array}$ $120.0 \mathrm{CM}: \quad 1.80$ 52.6

45. O CM: RHO PHI 20.0 CM: $1.92 \quad 44.9$

RHO PHI 45. O CM: 1.93 44.1
$0>[2.65 / 6.61 \mathrm{C} 0 / 1.5 / 4]$ 60.0 CM: $1.72 \quad 65.0$ 135.0 CM: 1.6363.

$0>[2.65 / 6.61 \mathrm{C} 0 / 1.5 / 4]$ $60.0 \mathrm{cM}: \quad 1.76 \quad 55$. $135.0 \mathrm{CM}: \quad 1.7952 .9$

$0>[2.65 / 6.61 \mathrm{C} 0 / 1.5 / 4]$ 60.0 cM: $\quad \begin{array}{rr}\text { RHO } & \text { PHI } \\ 1.86 & 48.6\end{array}$ $135.0 \mathrm{CM}: \quad 0.00 \quad 0.0$

$0>[2.65 / 6.61 \mathrm{C} \quad 0 / 1.5 / 4]$ $60.0 \mathrm{~cm}: \begin{array}{r}\mathrm{RHO} \\ \mathrm{PHI}\end{array}$ $135.0 \mathrm{CM}: \quad 1.77 \quad 54.2$

$0>[2.65 / 6.61 \mathrm{C} 0 / 1,5 / 4]$ 60 RHO PH 60.0 CM: 1.64 62.

$0>[2.65 / 6.61 \mathrm{C} 0 / 1.5 / 4]$ 60 RHO PH 35 O CM: 1.7754 .4

$0>[2.65 / 6.61 \mathrm{C} \quad 0 / 1.5 / 4]$

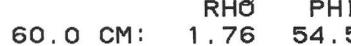
$135.0 \mathrm{CM}: 1.7455 .7$

$0>[2.65 / 6.61 \mathrm{C} 0 / 1.5 / 4]$ $60.0 \mathrm{CM}: 1.84 \quad 49$. $135.0 \mathrm{CM}: 1.8350 .6$

$0>[2.65 / 6.61 \mathrm{C} 0 / 1.5 / 4]$ 60.0 CM: $1.94 \quad 43.6$ 135.0 CM: 1.9344 .6

$0>[2.65 / 6.61 \mathrm{C} 0 / 1.5 / 4]$ $60.0 \mathrm{~cm}: \quad \begin{array}{rr}\mathrm{RHO} & \mathrm{PHI} \\ 1.95 & 43.2\end{array}$ 135. O CM: 1.9344 .2 
TABLE 1 - Continued

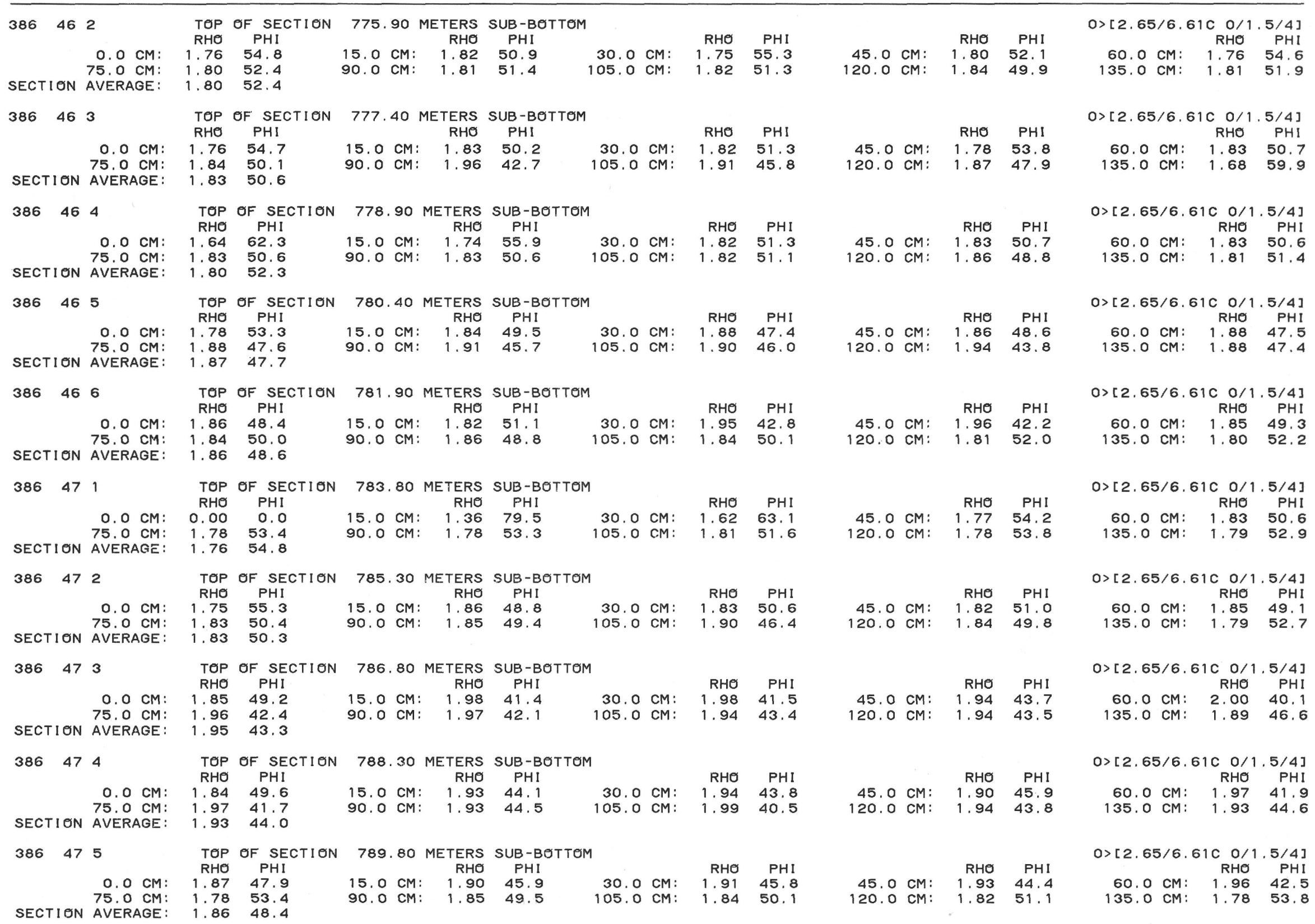


TOP OF SECTION 791.30 METERS SUB-BOTTOM

$\begin{array}{rrr}0.0 \mathrm{CM}: & \text { RHO } & \text { PHI } \\ 75.00 & 0.0\end{array}$

15. O CM: 1.80 PHI

90. $\mathrm{C}$ CM: $\quad 1.79 \quad 53.0$

30.0 CM: $\begin{array}{rr}\text { RHO } & \mathrm{PHI} \\ 1.82 & 51.2\end{array}$

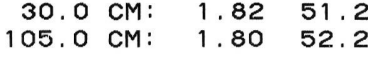
SECTION AVERAGE: 1.8151 .7

$386 \quad 48 \quad 2$

TOP OF SECTION 794.80 METERS SUB-BOTTOM

$\begin{array}{rrrr}0.0 & \mathrm{CM}: & 1.73 & 56.7 \\ 75.0 & \mathrm{CM}: & 1.72 & 57.3\end{array}$

SECTION AVERAGE: $1.78 \quad 53.4$

$386 \quad 483$

TOP OF SECTION 796.30 METERS SUB-BOTTOM

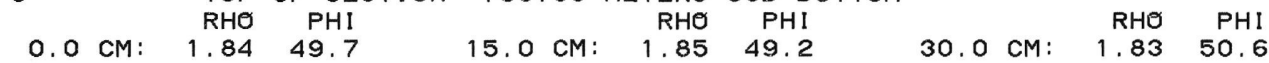

$\begin{array}{llllrlrr}0.0 & & \end{array}$

SECTION AVERAGE: $1.81 \quad 51.9$

$386 \quad 492$

TOP OF SECTION 804.30 METERS SUB-BOTTOM

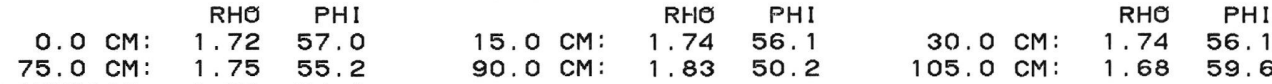

SECTION AVERAGE: $1.76 \quad 54.6$

$386 \quad 49$

TOP OF SECTION $\mathbf{8 0 5 . 8 0}$ METERS SUE-BOTTOM

RHO PHI RHO PHI RHO PHI

$\begin{array}{rrrrrrrrr}0.0 \mathrm{CM}: & 1.70 & 58.8 & 15.0 \mathrm{CM}: & 1.80 & 52.4 & 30.0 \mathrm{CM}: & 1.81 & 51.9 \\ 75.0 \mathrm{CM}: & 1.89 & 47.0 & 90.0 \mathrm{CM}: & 1.84 & 50.0 & 105.0 \mathrm{CM}: & 1.88 & 47.4\end{array}$

SECTION AVERAGE: 1.80 52.4

05. $0 \mathrm{CM}: 1.88 \quad 47.4$

$386 \quad 494$

TOP OF SECTION 807.30 METERS SUB-BOTTOM

$\begin{array}{rrrrrrrrr}0.0 \mathrm{CM}: & 1.82 & 50.9 & 15.0 \mathrm{CM}: & 1.76 & 54.9 & 30.0 \mathrm{CM}: & 1.87 & 48.0 \\ 75.0 \mathrm{CM}: & 1.92 & 45.0 & 90.0 \mathrm{CM}: & 1.89 & 46.5 & 105.0 \mathrm{CM}: & 1.88 & 47.4\end{array}$

SECTION 75.0 CM: $\quad 1.92 \quad 45.0$

$386 \quad 50 \quad 1$

TOP OF SECTION 812.30 METERS SUB-BOTTOM

$0.0 \mathrm{~cm}: \begin{array}{rr}\text { RHO } & \text { PHI } \\ 0.00 & 0.0\end{array}$

$75.0 \mathrm{CM}: \quad 1.84 \quad 49.6$

SECTION AVERAGE: $1.82 \quad 51.1$

15. $\mathrm{CM}: \mathrm{RHO} \quad \mathrm{PHI}$

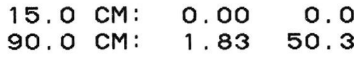

30.0 CM: $\quad$\begin{tabular}{rr}
$\mathrm{RHO}$ & $\mathrm{PHI}$ \\
\hline & 60.2
\end{tabular}

105. $\mathrm{CM}: \quad 1.87$ 47.9

$386 \quad 502$

TOP OF SECTION 813.80 METERS SUB-BOTTOM

RHO PHI

$\begin{array}{lll}0.0 \mathrm{CM}: & 1.65 & 61.7 \\ 75.0 \mathrm{CM}: & 1.81 & 51.6\end{array}$

SECTION AVERAGE: 1.8251 .3

RHO PHI

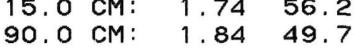

30.0 CM: $\quad 1.87 \quad 48.3$ 05.0 CM: 1.8648 .4

$386 \quad 503$

TOP OF SECTION 815.30 METERS SUB-BOTTOM

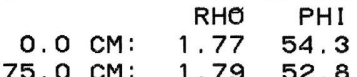

15.0 CM: 1.74 RH

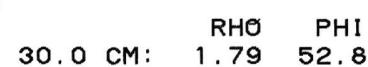

90.0 CM: $\quad 1.84 \quad 50.1$

105.0 CM: 1.8251 .3

SECTION AVERAGE: $\quad 1.8151 .8$

386504

TOP OF SECTION 816.80 METERS SUB-BOTTOM

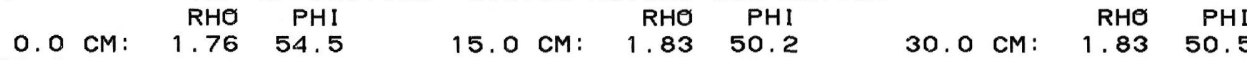

$1.83 \quad 50.4$
45.0 CM: $\quad \begin{array}{r}\mathrm{RHO} \\ \mathrm{PHI}\end{array}$

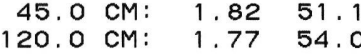

45. O CM: RHO PHI 120.0 CM: 1.88 47.4

45.0 CM: $\begin{array}{rr}\text { RHO } & \text { PHI } \\ 1.72 & 57.2\end{array}$

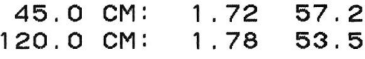

45. $\mathrm{RHO} \mathrm{PHI}$

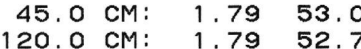

45.0 CM: $\quad \begin{array}{r}\text { RHO } \\ \text { PHI }\end{array}$

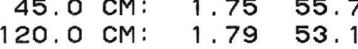

RHO PHI 120.0 CM: 1.88 47.3

45. $\mathrm{CM}: \quad \mathrm{RHO} \mathrm{PHI}$ $\begin{array}{rll}45.0 \mathrm{CM}: & 1.79 & 53.2 \\ 120.0 \mathrm{CM}: & 1.81 & 51.7\end{array}$

45.0 CM: $\begin{array}{rr}\text { RHO } & \text { PHI } \\ 1.88 & 47.6\end{array}$

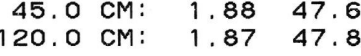

45. O CM: $\begin{array}{rr}\text { RHO } & \text { PHI } \\ 1.84 & 49.6\end{array}$

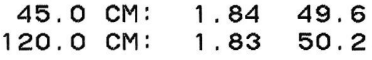

45. CM: RHO PHI 120.0 CM: 1.93 44.5
$0>[2.65 / 6.61 \mathrm{C} 0 / 1.5 / 4]$ $60.0 \mathrm{cM}: \quad \begin{array}{rr}\mathrm{RHO} & \mathrm{PHI} \\ 1.83 & 50.7\end{array}$ 135.0 CM: 1.8250 .9

$0>[2.65 / 6.61 \mathrm{C} 0 / 1.5 / 4]$ $60.0 \mathrm{cM}: \quad 1.82 \quad 50.8$ $135.0 \mathrm{CM}: \quad 1.81 \quad 51.5$

$0>[2.65 / 6.61 \mathrm{C} \quad 0 / 1.5 / 4]$ $60.0 \mathrm{~cm}: \quad \begin{array}{r}\mathrm{RHO} \\ 1.84\end{array}$ $135.0 \mathrm{CM}: 0.00 \quad 0.0$

O) $[2.65 / 6.61 \mathrm{C} \quad 0 / 1.5 / 4]$ $60.0 \mathrm{CM}: 1.8251 .3$

$0>[2.65 / 6.61 \mathrm{C} \quad 0 / 1.5 / 4]$ 60.0 CM: $\quad$ RHO PHI 135.0 CM: $1.75 \quad 55.3$

$0>[2.65 / 6.61 \mathrm{C} \quad 0 / 1.5 / 4]$ $60.0 \mathrm{cM}: \quad 1.85$ P8. 135.0 CM: $1.93 \quad 44.1$

$0>[2.65 / 6.61 \mathrm{C} 0 / 1.5 / 4]$ 60.0 CM: 1.85 49.4 $135.0 \mathrm{CM}: \quad 1.7456 .1$

$0>[2.65 / 6.61 \mathrm{C} \quad 0 / 1.5 / 4]$ $60.0 \mathrm{cM}: \quad \begin{array}{rr}\mathrm{RHO} & \mathrm{PHI} \\ 1.76 & 54.7\end{array}$ 135.0 CM: 1.90 46.1

$0>[2.65 / 6.61 \mathrm{C} \quad 0 / 1.5 / 4]$

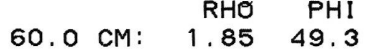
135.0 CM: $1.82 \quad 51.3$

$0>[2.65 / 6.61 \mathrm{C} 0 / 1.5 / 4]$ RHO PHI

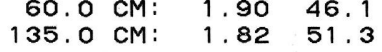


$386 \quad 505$ TOP OF SECTION $\mathbf{8 1 8 . 3 0}$ METERS SUB-BOTTOM $0.0 \mathrm{CM}:$
$75.0 \mathrm{CM}:$ RHO PHI $\begin{array}{lll}75.0 \mathrm{CM}: & 1.88 & 47.2 \\ \text { AVERAGE: } & 1.84 & 49.9\end{array}$ $\begin{array}{rrr}15.0 \mathrm{CM}: & 1.82 & 51.2 \\ 90.0 \mathrm{CM}: & 1.87 & 47.8\end{array}$ $30.0 \mathrm{cM}$
$105.0 \mathrm{cM}$

\section{RHO $\mathrm{PHI}$} SECTION AVERAGE:

TOP OF SECTION 822.34 METERS SUB-BOTTOM $\begin{array}{lll}386 & 51 & 1\end{array}$

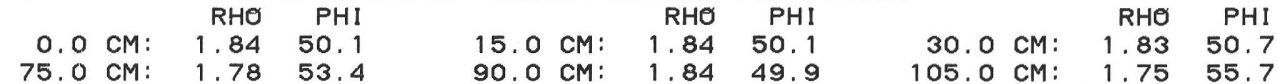
SECTIEN AVERAGE: 1.8053 .4

$\begin{array}{lll}386 & 512\end{array}$

TOP OF SECTION 823.84 METERS SUB-BOTTOM

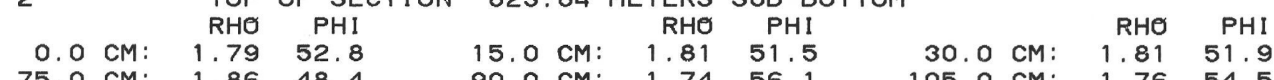
SECTION 75.0 CM: $\quad 1.86 \quad 48.4$

$\begin{array}{lll}386 & 513\end{array}$

\section{TOP OF SECTION 825.34 METERS SUB-BOTTOM}

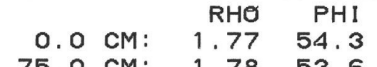
15. $\mathrm{CM}$ RHO PHI $\begin{array}{lll}75.0 \mathrm{CM}: & 1.78 & 53.6 \\ \text { AVERAGE: } & 1.81 & 51.6\end{array}$ SECTION AVERAGE:

$\begin{array}{lll}386 & 514\end{array}$

TOP OF SECTION 826.84 METERS SUB-BOTTOM

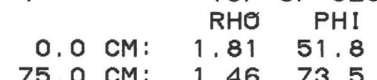
$\begin{array}{lll}75.0 \mathrm{CM}: & 1.46 & 73.5 \\ \text { AVERAGE: } & 1.79 & 52.7\end{array}$ SECTION AVERAGE: $1.79 \quad 52.7$

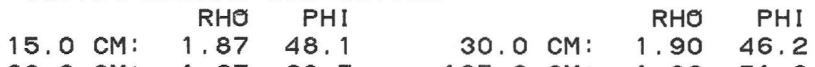
90.0 CM: 1.67 60.5 105.0 cM: $1.82 \quad 51.0$

$386 \quad 515$

TOP OF SECTION 828.34 METERS SUB-BOTTOM

$\begin{array}{rr}\mathrm{RHO} & \mathrm{PHI} \\ 1.78 & 53.4\end{array}$

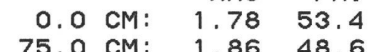
15.0 $\mathrm{RHO}$ PHI $\begin{array}{lll}75.0 \mathrm{CM}: & 1.86 & 48.6 \\ \text { SECTION AVERAGE: } & 1.81 & 51.5\end{array}$ 90.0 CM: 1.75 55.2 $105.0 \mathrm{CM}: 1.8052 .2$ $386 \quad 52$

TOP OF SECTION 831.40 METERS SUB-BOTTOM

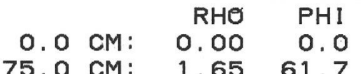
$\begin{array}{lll}75.0 \text { CM: } & 1.65 & 61.7 \\ \text { SECTION AVERAGE: } & 1.78 & 53.8\end{array}$

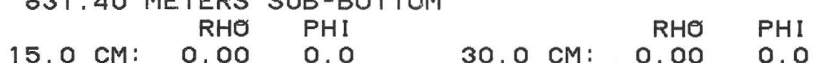

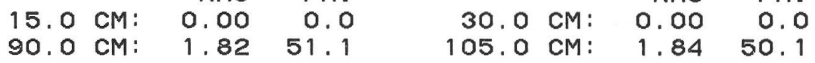
$386 \quad 522$

TOP OF SECTION 832.90 METERS SUB-BOTTOM

$\begin{array}{rrr}0.0 \mathrm{CM}: & \text { RHO } & \mathrm{PHI} \\ 75.0 \mathrm{CM} & 1.51 & 53.2 \\ 70.2\end{array}$

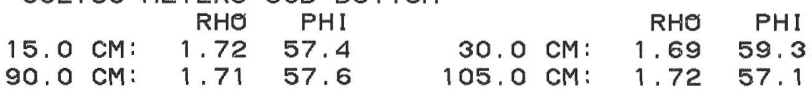
$386 \quad 523$

TOP OF SECTION 834.40 METERS SUB-BOTTOM

386523

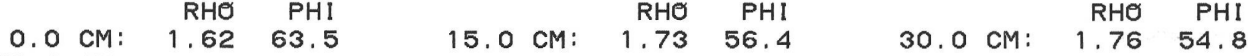
SECTION $75.0 \mathrm{CM}: \quad 1.7953 .1$

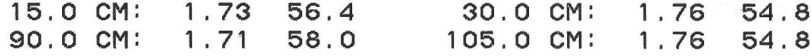
$386 \quad 524$

TOP OF SECTION 835.90 METERS SUB-BOTTOM

386524

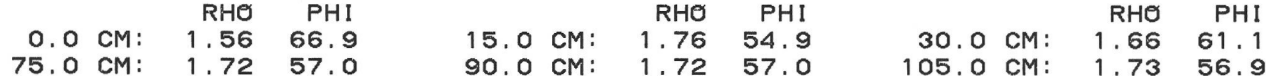
SECTION AVERAGE: $1.72 \quad 57.0$
45. $\mathrm{CM}: \quad \begin{array}{r}\mathrm{RHO} \\ \mathrm{PHI}\end{array}$

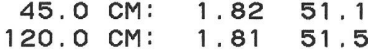
45. $\mathrm{CM}:$ RHO PHI

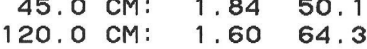
45. $\mathrm{RHO} \mathrm{PHI}$

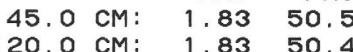
45. $\mathrm{CM}: \mathrm{RHO} \mathrm{PHI}$

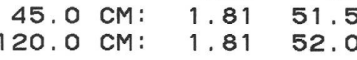

45. O CM: $\begin{array}{rr}\text { RHO } & \text { PHI } \\ 1.92 & 44.9\end{array}$ $120.0 \mathrm{CM}: \quad 1.83$ 50.3

45. O CM: \begin{tabular}{rr} 
RHO & PHI \\
\hline & 030.2
\end{tabular} 120.0 CM: 1.7952 .8

45. $\mathrm{CM}: \mathrm{RHO} \quad \mathrm{PHI}$

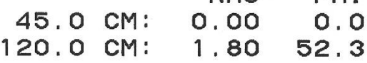

45. $\mathrm{RHO}$ PHI

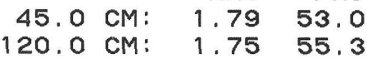

$45.0 \mathrm{CM}$ RHO PHI

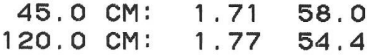

45.0 CM: $\quad \begin{array}{r}\text { RHO } \\ \text { PHI }\end{array}$

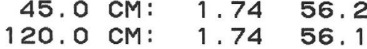

$0>[2.65 / 6.61 \mathrm{C} 0 / 1.5 / 4]$ 60.0 CM: 1.87 R7.9

$0>[2.65 / 6,61 \mathrm{C} 0 / 1,5 / 4]$ $60 \mathrm{RHO} P \mathrm{PHI}$

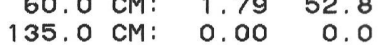

$0>[2.65 / 6.61 \mathrm{C} 0 / 1.5 / 4]$

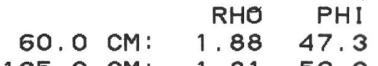
$135.0 \mathrm{CM}: 1.8152 .0$

o) $[2.65 / 6.61 \mathrm{C} 0 / 1.5 / 4]$

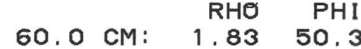
$135.0 \mathrm{cM}: 1.8251 .0$

$0>[2.65 / 6.61 \mathrm{C} 0 / 1.5 / 4]$ $60.0 \mathrm{cM}: \quad 1.85$ RH $135.0 \mathrm{cM}: 1.8251 .1$

$0>[2.65 / 6.61 \mathrm{C} \quad 0 / 1.5 / 4]$

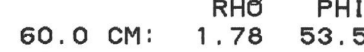
135.0 CM: $1.80 \quad 52.5$

$0>[2.65 / 6.61 \mathrm{C} 0 / 1.5 / 4]$ $60.0 \mathrm{~cm}: \quad \begin{array}{r}\mathrm{RHO} \\ \quad \mathrm{PHI}\end{array}$ $135.0 \mathrm{CM}: 1.84 \quad 49.5$

$0>[2.65 / 6.61 \mathrm{C} 0 / 1.5 / 4]$ 60.0 CM: $1.72 \quad 57.0$

$0>[2.65 / 6.61 \mathrm{C} 0 / 1.5 / 4]$ 60.0 CM: $1.74 \quad 55.8$ $0>[2.65 / 6.61 \mathrm{C} \quad 0 / 1.5 / 4]$ 60.0 CM: $1.66 \quad 61.0$ 
$386 \quad 525$

TOP OF SECTION 837.40 METERS SUB-BOTTOM

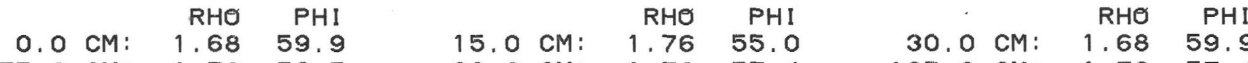

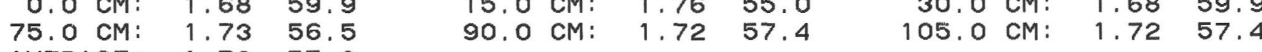

SECTION AVERAGE: $1.72 \quad 57.3$

TOP OF SECTION 838.90 METERS SUB-BOTTOM

386526

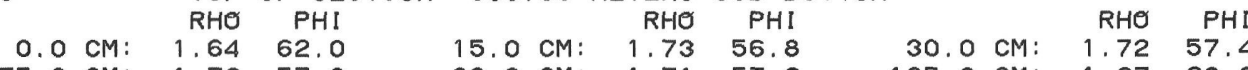

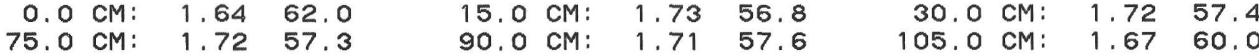

SECTION AVERAGE: 1.6959 .3

TOP OF SECTION $\mathbf{8 4 0 . 9 0}$ METERS SUB-BOTTOM

$\begin{array}{lll}386 & 53 & 1\end{array}$

\begin{tabular}{|c|c|c|c|c|c|c|c|c|c|c|}
\hline & RHO & $\mathrm{PHI}$ & & & RHO & $\mathrm{PHI}$ & & & RHO & \\
\hline $0.0 \mathrm{C}$ & $\begin{array}{l}0.00 \\
1.78\end{array}$ & $\begin{array}{r}0.0 \\
53.3\end{array}$ & 15.0 & CM: & 0.00 & 0.0 & $\begin{array}{r}30.0 \\
05\end{array}$ & CM: & 0.00 & 54 \\
\hline
\end{tabular}

SECTION AVERAGE: $1.77 \quad 54.4$

$386 \quad 532$

TOP OF SECTION $\mathbf{8 4 2 . 4 0}$ METERS SUB-BOTTOM

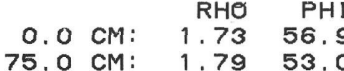

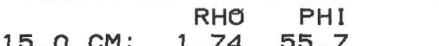

$\begin{array}{lll}75.0 \mathrm{CM}: & 1.79 & 53.0 \\ \text { AVERAGE: } & 1.78 & 53.5\end{array}$

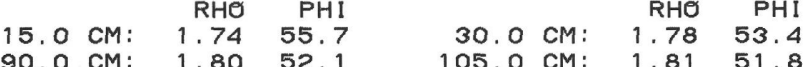

TOP OF SECTION 843.90 METERS SUB-BOTTOM

$386 \quad 53 \quad 3$

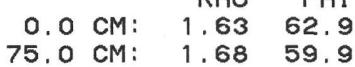

$\begin{array}{rrr}15.0 \mathrm{CM}: & \text { RHO } & \text { PHI } \\ & 1.72 & 57.0\end{array}$

30.0 CM: 1.62 PHI

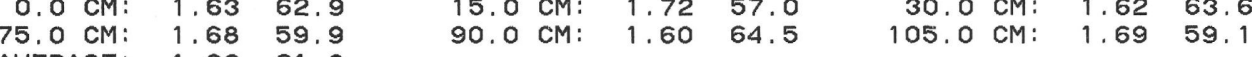

SECTION AVERAGE: 1.66 61.0

$\begin{array}{lll}386 & 53 \quad 4\end{array}$

$0.0 \mathrm{CM}:$
$75.0 \mathrm{CM}:$

TOP OF SECTION 845.40 METERS SUB-BOTTOM

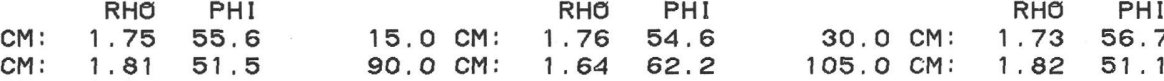
SECTION AVERAGE: $1.77 \quad 54.3$

$386 \quad 54 \quad 1$

$$
\begin{array}{lll}
\text { TOP OF SECTION } 850.65 \text { METERS SUB-BOTTOM } \\
\text { RHO PHI }
\end{array}
$$

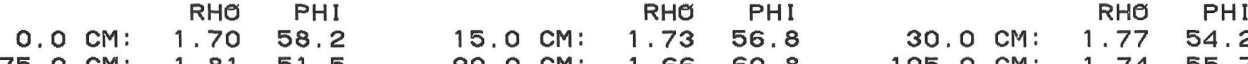

SECTION AVERAGE: $\quad 1.8151 .5$

$\begin{array}{lll}386 & 542\end{array}$

TOP OF SECTION $\mathbf{8 5 2 . 1 5}$ METERS SUB-BOTTOM

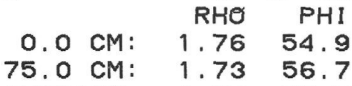

15. cM: RHO PHI 1 30.0 cM: RHO PHI

$90.0 \mathrm{CM}: 1.75$ 55.3 $105.0 \mathrm{CM}: 1.66$ 60.8

SECTION AVERAGE:

$386 \quad 543$

TOP OF SECTION 853.65 METERS SUB-BOTTOM

$\mathrm{RHO} \mathrm{PHI}$

0.0 CM: $\quad 1.73 \quad 56.6$

$\begin{array}{rrr}15.0 \mathrm{CM}: & \mathrm{RHO} & \mathrm{PHI} \\ 1.68 & 59.6\end{array}$

$\begin{array}{lll}75.0 \mathrm{CM}: & 1.65 & 61.5 \\ \text { AVERAGE: } & 1.72 & 57.2\end{array}$

$90.0 \mathrm{CM}: 1.75 \quad 55.2$

$30.0 \mathrm{~cm}: 1.74$ PHI

30.0 CM: $1.74 \quad 55.8$

TOP OF SECTION 855.15 METERS SUB-BOTTOM

$386 \quad 54 \quad 4$

$\begin{array}{rrr}0.0 \mathrm{CM}: & \text { RHO } & \mathrm{PHI} \\ & 1.65 & 61.4\end{array}$

75.0 CM: $\quad 1.72 \quad 57.4$

$\begin{array}{lll}15.0 & \mathrm{CM}: \quad \mathrm{RHO} & \mathrm{PHI} \\ & 1.74 & 55.7\end{array}$

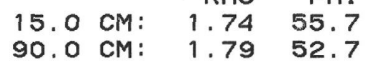

30.0 CM: $\quad \begin{array}{rr}\text { RHO } & \mathrm{PHI}\end{array}$

$\begin{array}{rrr}30.0 \mathrm{CM}: & 1.76 & 55.0 \\ 105.0 \mathrm{CM}: & 1.66 & 60.7\end{array}$

45. O CM: $\begin{array}{rrr}\text { RHO } & \text { PHI } \\ 1.71 & 57.6\end{array}$

RHO PHI

45. O CM: $\quad$ RHO PHI SECTION AVERAGE: $1.72 \quad 57.5$ 120.0 CM: 1.7654 .8

45. $\mathrm{RHO} \quad \mathrm{PHI}$ $120.0 \mathrm{CM}: 1.6859 .9$

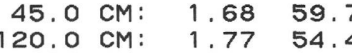

45. $\mathrm{RH}: \mathrm{PH}$

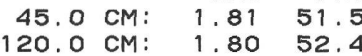

1.80 .02

45. O CM: RHO PHI

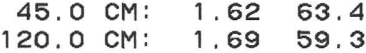

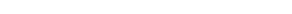

45.0 CM: RHO PHI

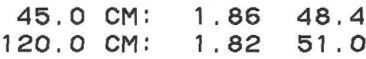

45. O CM: $\quad \begin{array}{rr}\text { RHO } & \text { PHI } \\ 1.77 & 54.2\end{array}$ $\begin{array}{rlll}\text { 45. O CM: } & 1.77 & 54.2 \\ 120: 0 & \text { CM: } & 1.77 & 54.4\end{array}$ 120.0 CM: 1.7256 .6

45.0 CM: $\quad \begin{array}{rr}\text { RHO } & \text { PHI } \\ 1.75 & 55.3\end{array}$ $120.0 \mathrm{cM}: \quad 1.70 \mathrm{58} .7$

$0>[2.65 / 6.61 \mathrm{C} 0 / 1.5 / 4]$ $\begin{array}{rrrr}60.0 & \mathrm{CM}: & \mathrm{RHO} & \mathrm{PHI} \\ 1351 & 57.7\end{array}$ 135.0 CM: $1.72 \quad 57.2$

$0>[2,65 / 6,61 \mathrm{C} 0 / 1,5 / 4]$ $60.0 \mathrm{cM}: \quad \begin{array}{r}\mathrm{RHO} \\ 1.69\end{array}$ $135.0 \mathrm{CM}: \quad 1.56 \quad 66.8$

$0>[2.65 / 6.61 \mathrm{C} \quad 0 / 1.5 / 4]$ $60.0 \mathrm{CM}: 1.78 \quad 53.5$ 135.0 CM: $1.77 \quad 54.0$

$0>[2.65 / 6.61 \mathrm{C} \quad 0 / 1.5 / 4]$

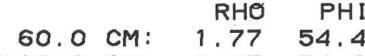
$135.0 \mathrm{CM}: 1.7754$.

$0>[2.65 / 6.61 \mathrm{C} 0 / 1.5 / 4]$ 60.0 CM: 1.63 62.6 $0>[2.65 / 6.61 \mathrm{C} 0 / 1.5 / 4]$

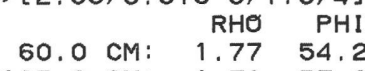
135.0 CM: 1.7157 .8

$0>[2.65 / 6.61 \mathrm{C} 0 / 1.5 / 4]$

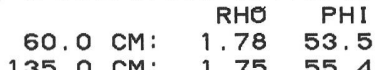
$0>[2.65 / 6.61 \mathrm{C} \quad 0 / 1.5 / 4]$

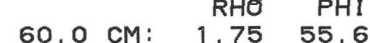
135.0 CM: $1.78 \quad 53.4$

$0>[2.65 / 6.61 \mathrm{C} 0 / 1.5 / 4]$ 60.0 CM: RHO PHI 135.0 CM: 1.7257 .0

$0>[2.65 / 6.61 \mathrm{C} \quad 0 / 1.5 / 4]$

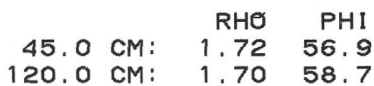




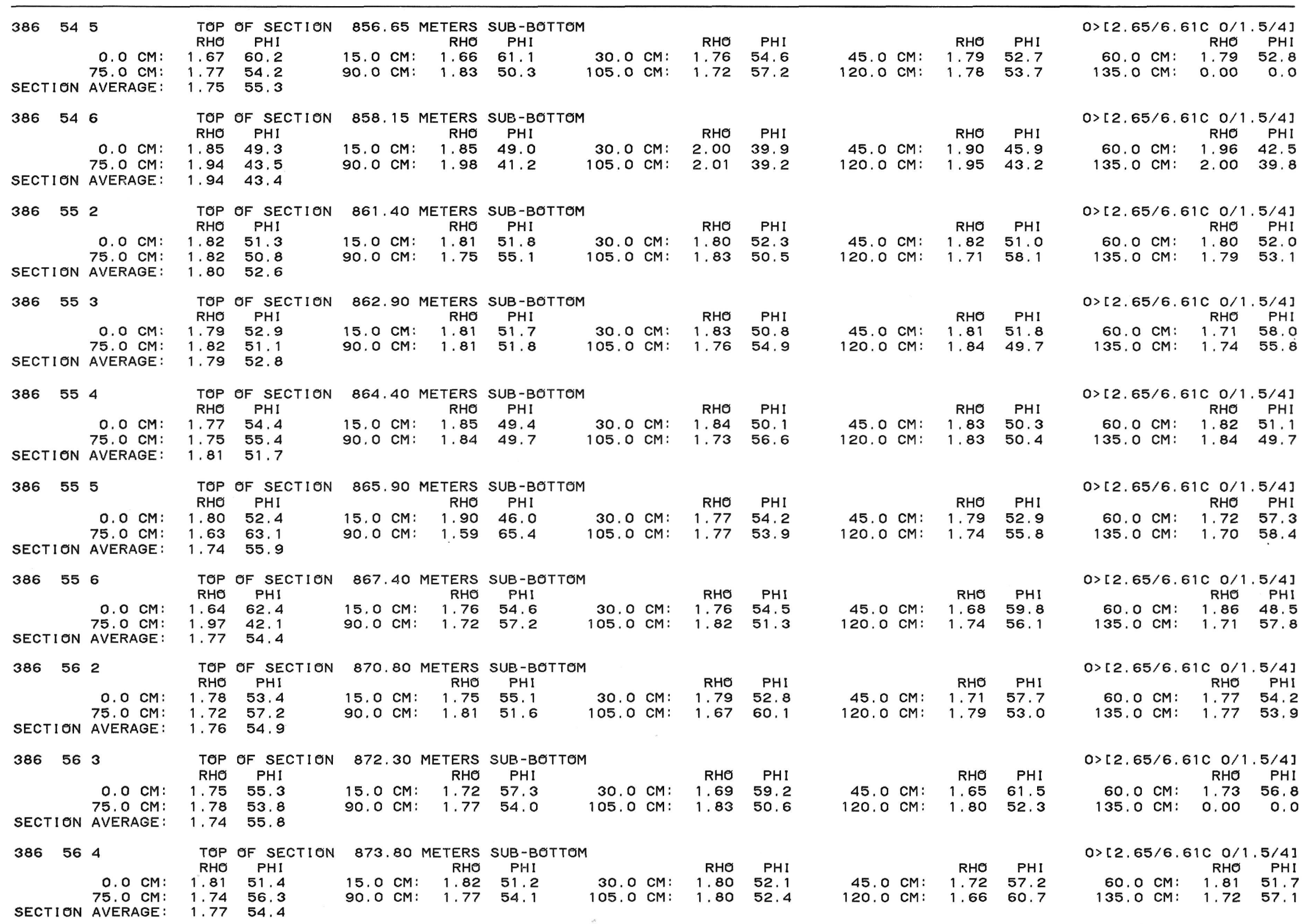


TOP OF SECTION 875.30 METERS SUB-BOTTOM

0.0 CM: $\begin{array}{rr}\text { RHO } & \text { PHI } \\ 7.67 & 60.0\end{array}$

$75.0 \mathrm{CM}: 1.6164 .1$

SECTION AVERAGE: 1.6959 .1

RHO PHI

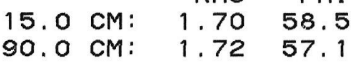

30. O CM: $\begin{array}{rr}\text { RHO } & \text { PHI } \\ 1.69 & 59.0\end{array}$

$105.0 \mathrm{CM}: 1.70 \quad 58.5$

TOP OF SECTION 880.40 METERS SUB-BOTTOM

$386 \quad 572$

RHO PHI

$\begin{array}{rrr}0.0 \mathrm{CM}: & 1.76 & 54.6 \\ 75.0 \mathrm{CM}: & 1.78 & 53.5\end{array}$

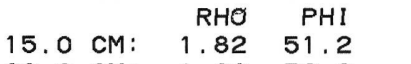

30.0 CM: $\begin{array}{rr}\text { RHO } & \text { PHI } \\ 1.80 & 52.5\end{array}$ 90.0 CM: 1.80 52.0 $105.0 \mathrm{CM}: 1.7157 .9$

SECTION AVERAGE: 1.7753 .9

$386 \quad 573$

TOP OF SECTION 881.90 METERS SUB-BOTTOM

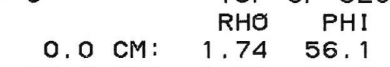

$\begin{array}{lll}75.0 \mathrm{CM}: & 1.82 & 51.2 \\ \text { SECTION AVERAGE: } & 1.80 & 52.5\end{array}$

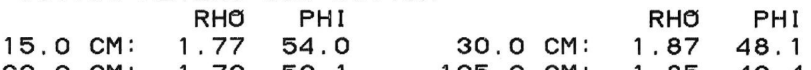

90.0 CM: 1.79 53.1 $105.0 \mathrm{CM}: 1.85 \quad 49.4$

$386 \quad 57 \quad 4$

TOP OF SECTION 883.40 METERS SUB-BOTTOM

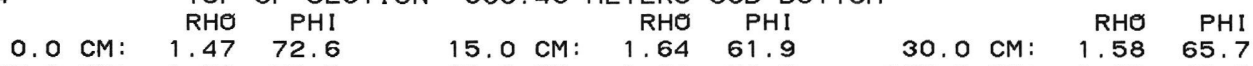

$\begin{array}{lllllll} & & \end{array}$

SECTION AVERAGE: 1.7157 .6

$386 \quad 575$

TOP OF SECTION 884.90 METERS SUB-BOTTOM

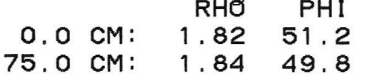

15.0 CM: 1.86 PHI

$\begin{array}{lll}15.0 \mathrm{CM}: & 1.86 & 48.5 \\ 90.0 \mathrm{CM}: & 1.85 & 49.2\end{array}$

30.0 CM: $\begin{array}{rrr}\mathrm{RHO} & \mathrm{PHI} \\ 105.0 \mathrm{CM} & 1.83 & 50.5\end{array}$

$1.84 \quad 49.8$

SECTION AVERAGE:

TOP OF SECTION 886.40 METERS SUB-BOTTOM

$386 \quad 57 \quad 6$

$\begin{array}{rrr}0.0 \mathrm{CM}: & 1.78 & 53.8 \\ 75.0 \mathrm{CM}: & 1.75 & 55.6\end{array}$

$\begin{array}{rrr}15.0 \mathrm{CM}: & \mathrm{RHO} & \mathrm{PHI} \\ 1.74 & 55.7\end{array}$

30.0 CM: $\quad \begin{array}{r}\mathrm{RHO} \\ \mathrm{PHI}\end{array}$

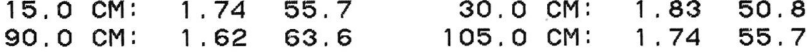

SECTION AVERAGE: $1.75 \quad 55.6$

$\begin{array}{lll}386 & 58 & 1\end{array}$

TOP OF SECTION 888.52 METERS SUB-BOTTOM

$0.0 \mathrm{cM}: \mathrm{RHO} \mathrm{PHI}$

$\begin{array}{lll}0.0 \mathrm{CM}: & 1.80 & \mathbf{5 2 . 5} \\ 75.0 \mathrm{CM}: & 1.61 & 63.8\end{array}$

SECTION AVERAGE: 1.6959 .4

$15 \mathrm{~cm}$ RHO PHI $1.8 \mathrm{~cm}$ RHO PHI

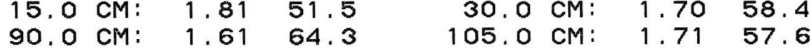

$386 \quad 582$

TOP OF SECTION $\mathbf{8 9 0 . 0 2}$ METERS SUB-BOTTOM

$\begin{array}{rrr}0.0 \mathrm{CM}: & \text { RHO } & \text { PHI } \\ 75.71 & 57.9\end{array}$

$\begin{array}{lll}75.0 \mathrm{CM}: & 1.69 & \mathbf{5 8 . 8} \\ \text { SECTION AVERAGE: } & 1.68 & 59.7\end{array}$

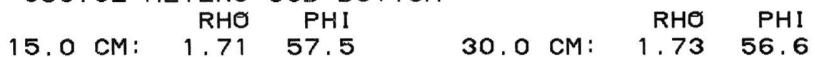

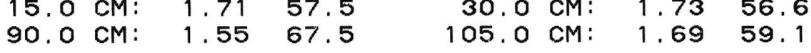

$386 \quad 58 \quad 3$

\section{TOP OF SECTION 891.52 METERS SUB-BOTTOM}

RHO PHI

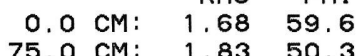

15.0 CM: 1.77 PHI

$\begin{array}{lll}\text { 75. O CM: } & 1.83 & 50.3 \\ \text { AVERAGE: } & 1.79 & 52.8\end{array}$

386584

TOP OF SECTION 893.02 METERS SUB-BOTTOM

RHO PHI

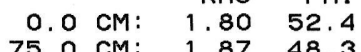

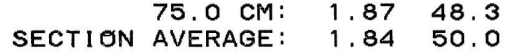

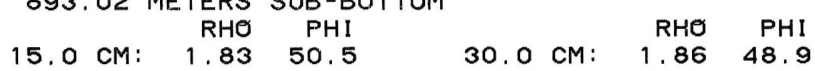

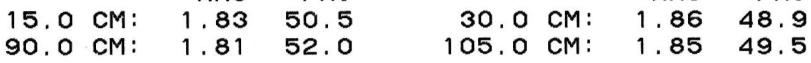

45. $\mathrm{CM}: \mathrm{RHO} \mathrm{PHI}$ $\begin{array}{lll}420.0 \mathrm{CM}: & 1.67 & 60.2 \\ & 1.73 & 56.6\end{array}$

45. O CM: $\begin{array}{rrr}\text { RHO } & \mathrm{PHI} \\ 1.80 & 52.2\end{array}$ 120.0 CM: 1.6760 .1

45. O cM: $\begin{array}{rr}\text { RHO } & \mathrm{PHI} \\ 1.82 & 51.4\end{array}$ $120.0 \mathrm{cM}: \quad 1.67$ 60.5

$45.0 \mathrm{CM}, \mathrm{RHO} \quad \mathrm{PHI}$

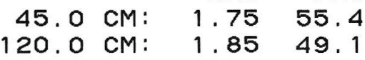

$45.0 \mathrm{cM}: \quad \mathrm{RHO} \mathrm{PHI}$

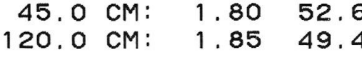

45.0 CM: $\begin{array}{rrr}\text { RHO } & \text { PHI } \\ 1.71 & 57.9\end{array}$ $120.0 \mathrm{CM}: 1.69 \quad 59.4$

45.0 $\mathrm{CM}: \quad \mathrm{RHO} \quad \mathrm{PHI}$ $\begin{array}{lll}\text { 45.0 CM: } & 1.56 & 67.1 \\ 20.0 \mathrm{CM}: & 1.78 & 53.8\end{array}$

45. $\mathrm{CM} \quad \mathrm{RHO} \mathrm{PHI}$

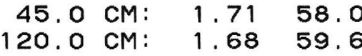

45.0 CM: $\begin{array}{rr}\text { RHO } & \text { PHI } \\ 1.75 & 55.6\end{array}$ 120.0 CM: 1.85 49.2

45. $\mathrm{RMO}$ PHI $\begin{array}{rrr}45.0 \mathrm{CM}: & 1.86 & 48.5 \\ 120.0 \mathrm{CM}: & 1.86 & 48.8\end{array}$
$0>[2.65 / 6.61 \mathrm{C} 0 / 1.5 / 4]$ $60.0 \mathrm{cM}: \quad \begin{array}{rr}\text { RHO } \\ 1.64 & \mathrm{PHI}\end{array}$ $135.0 \mathrm{~cm}: 1.7754 .0$

$0>[2.65 / 6.61 \mathrm{C} \quad 0 / 1.5 / 4]$ $60.0 \mathrm{cM}: \quad 1.84 \quad 50.0$ $135.0 \mathrm{CM}: 1.76 \quad 54.8$

O) $[2.65 / 6.61 \mathrm{C} \quad 0 / 1.5 / 4]$

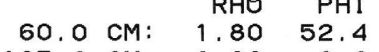
$135.0 \mathrm{CM}: \quad 0.00 \quad 0.0$

$0>[2.65 / 6.61 \mathrm{C} 0 / 1.5 / 4]$

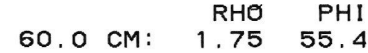
135. O CM: $1.71 \quad 57.7$

$0>[2.65 / 6.61 \mathrm{C} 0 / 1.5 / 4]$

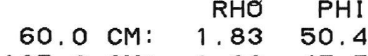
135. O CM: $1.88 \quad 47.5$

$0>[2.65 / 6.61 \mathrm{C} 0 / 1.5 / 4]$

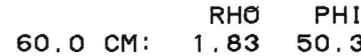
$135.0 \mathrm{CM}: \quad 1.8251 .1$

$0>[2.65 / 6.61 \mathrm{C} \quad 0 / 1.5 / 4]$ 60.0 CM: $\begin{array}{rr}\text { RHO } & \text { PHI } \\ 1.62 & 63.5\end{array}$ $0>[2.65 / 6.61 \mathrm{C} 0 / 1.5 / 4]$ $60.0 \mathrm{~cm}: \quad \begin{array}{r}\mathrm{RHO} \\ \mathrm{PH} 1\end{array}$ 135.0 CM: 1.64 61.9

$0>[2.65 / 6.61 \mathrm{C} 0 / 1.5 / 4]$

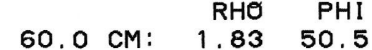
$135.0 \mathrm{CM}: 1.84 \quad 49.9$ $0>[2.65 / 6.61 \mathrm{C} 0 / 1.5 / 4]$ $60.0 \mathrm{cM}: \quad \begin{array}{r}\mathrm{RHO} \\ \mathrm{PHI}\end{array}$ 135.0 CM: $1.86 \quad 48.5$ 


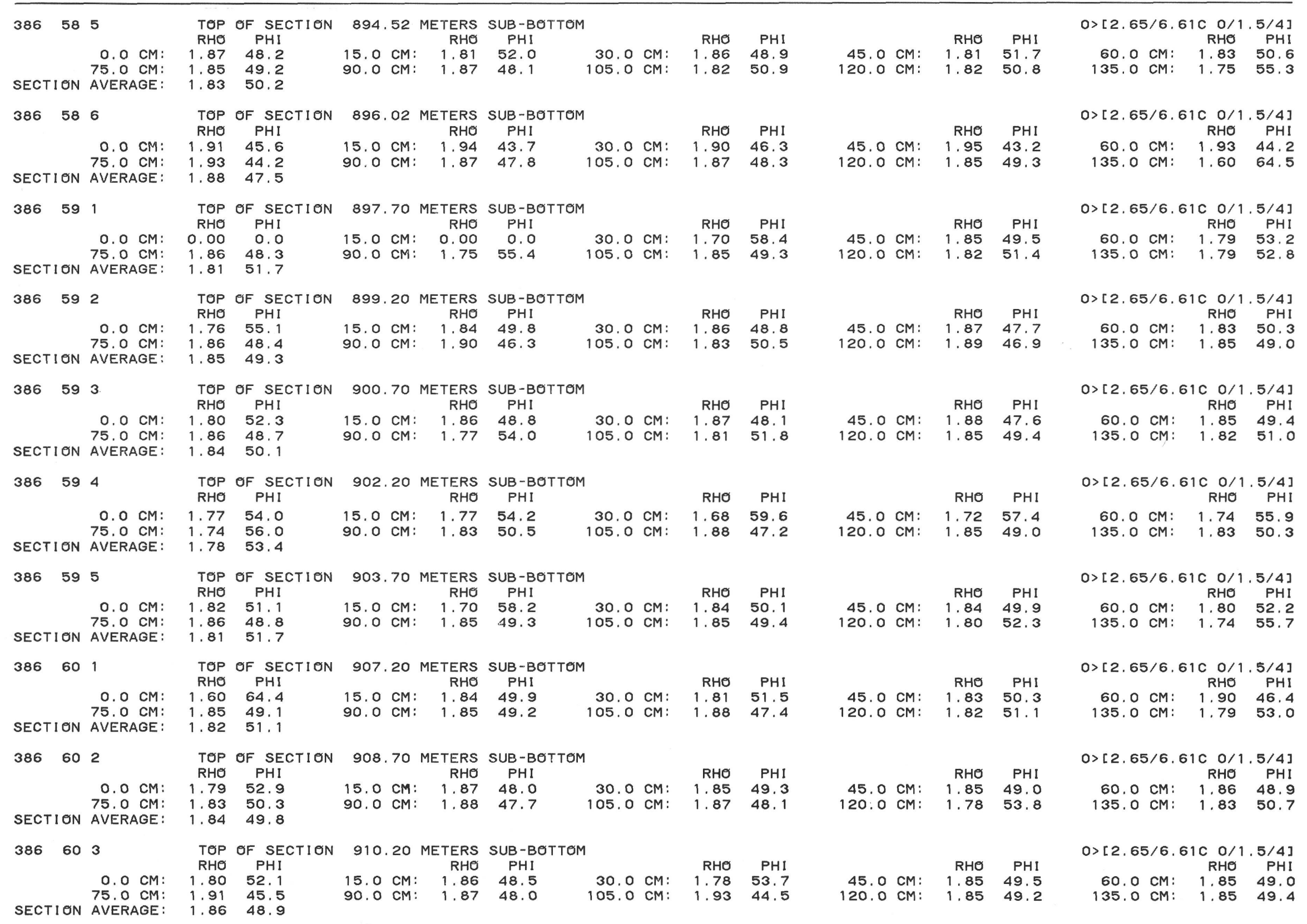


$386 \quad 60 \quad 4$

OF SECTION 911.70 METERS SUB-BOTTOM

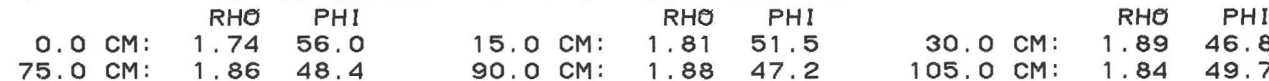

SECTION AVERAGE: 1.85 . 49.1

$386 \quad 605$

TOP OF SECTION 913.20 METERS SUB-BOTTOM

$0.0 \mathrm{CM}: \quad 1.77,54.4$

SECTION AVERAGE: 1.8549 .0

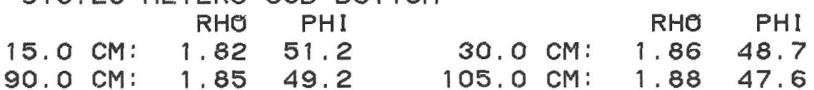

05.0 CM: $1.88 \quad 47.6$

386606

TOP OF SECTION 914.70 METERS SUB-BOTTOM

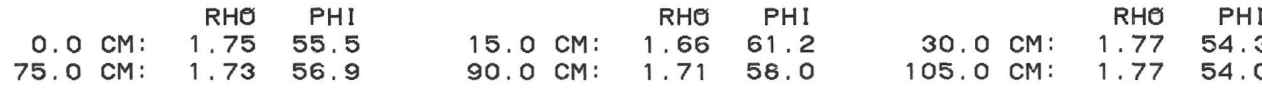

SECTION AVERAGE: $1.73 \quad 56.5$

$\begin{array}{lll}386 & 61 & 1\end{array}$

TOP OF SECTION 916.80 METERS SUB-BOTTOM

RHO PHI

$0.0 \mathrm{CM}: \quad 1.48 \quad 72.0$

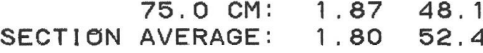

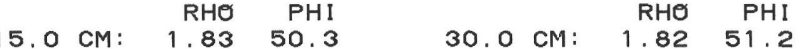
90.0 CM: $1.86 \quad 48.5 \quad 105.0 \mathrm{CM}: 1.7158 .0$

$386 \quad 612$

TOP OF SECTION 918.30 METERS SUB-BOTTOM

918.30 METERS SUB-BOTTOM
RHO PHI

O.O CM: $\quad 1.64 \quad 62.1$

SECTION AVERAGE: 1.7555 .2

$386 \quad 613$

TOP OF SECTION 919.80 METERS SUB-BOTTOM

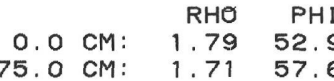

SECTION AVERAGE: 1.7157 .8

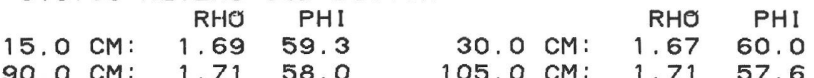

105.0 CM: 1.7157 .6

$386 \quad 61 \quad 4$

TOP OF SECTION 921.30 METERS SUB-BOTTOM

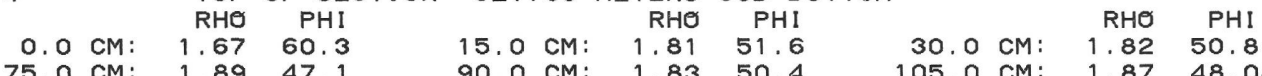

SECTION AVERAGE: 1.85 49.1

$\begin{array}{lll}386 & 615\end{array}$

TOP OF SECTION 922.80 METERS SUB-BOTTOM

RHO PHI RHO PHI RHO PHI

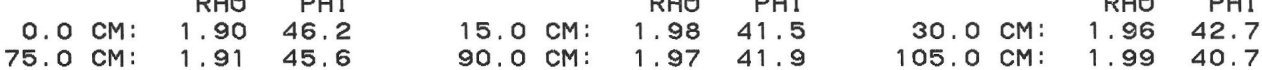

SECTION AVERAGE: $1.94 \quad 43.8$

$386 \quad 622$

TOP OF SECTION 927.80 METERS SUB-BOTTOM

$0.0 \mathrm{CM}: \quad 1.75 \quad 55.1$

RHO PHI

$30.0 \mathrm{cM}: \quad \mathrm{RHO} \mathrm{PHI}$

75. O CM: $\quad 1.80 \quad 52.4$

90. $0 \mathrm{CM}: 1.75 \quad 55.3$

$\begin{array}{lll}105.0 \mathrm{CM}: & 1.75 & 55.4 \\ & 1.67 & 60.5\end{array}$

SECTION AVERAGE: $1.78 \quad 53.7$

$386 \quad 623$

TOP OF SECTION 929.30 METERS SUB-BOTTOM

0.0 CM: $\quad \begin{array}{rr}\text { RHO } & \text { PHI } \\ 75.73 & 56.4\end{array}$

$75.0 \mathrm{CM}: \quad \begin{array}{lll}1.85 & 49.3\end{array}$

SECTION AVERAGE: 1.8151 .6 $\begin{array}{llllll}15.0 \mathrm{cM}: & 1.81 & 51.5 & 30.0 \mathrm{CM}: & 1.83 & 50.5\end{array}$ 90.0 CM: 1.77 53.9 $105.0 \mathrm{cM}: \quad 1.85$ 49.1

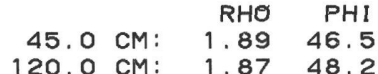

45. $\mathrm{CM}:$ RHO PHI

$20.0 \mathrm{CM} \quad 1.82 \quad 45.5$

$\begin{array}{rrr} & \text { RHO } & \text { PHI } \\ \text { 45. } & \text { CM: } \quad 1.80 & 52.6\end{array}$

120.0 CM: $1.70 \quad 58.5$

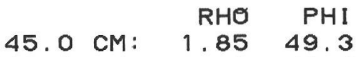
120.0 CM: 1.8648 .4

45. $\mathrm{CM}$ RHO PHI $\begin{array}{rll}420.0 \mathrm{CM}: & 1.70 & 58.6 \\ & & \end{array}$

45.0 CM: $\begin{array}{rr}\text { RHO } & \text { PHI } \\ 1.71 & 58.1\end{array}$ 20.0 CM: 1.7157 .8

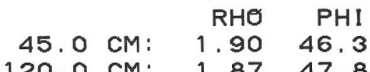

45. $\mathrm{CM}: \mathrm{RHO} \mathrm{PHI}$

120.0 CM: 1.9145 .4

45. O CM: $\quad \begin{array}{r}\text { RHO PHI } \\ \text { PH }\end{array}$ $120.0 \mathrm{cM}: \quad 1.80 \quad 52.1$

45. $\mathrm{CM}: \quad \begin{array}{r}\mathrm{RHO} \\ 1.81\end{array}$ 120.0 CM: 1.8250 .9
$0>[2.65 / 6.61 \mathrm{C} 0 / 1.5 / 4]$ 60.0 CM: 1.9046 .3 35. $\mathrm{O} \mathrm{CM}: 1.8350 .7$

$0>[2.65 / 6.61 \mathrm{C} 0 / 1.5 / 4]$ $60.0 \mathrm{cM}: \quad \begin{array}{rr}\mathrm{RHO} & \mathrm{PHI}\end{array}$ $135.0 \mathrm{CM}: 0.00 \quad 0.0$

$0>[2.65 / 6.61 \mathrm{C} 0 / 1.5 / 4]$

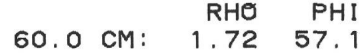
$135.0 \mathrm{CM}: \quad 1.72 \quad 57.1$

$0>[2.65 / 6.61 \mathrm{C} 0 / 1.5 / 4]$ $60.0 \mathrm{cM}: \quad \begin{array}{rr}\mathrm{RHO} & \mathrm{PHI}\end{array}$ 135.0 CM: 1.83 50.7

$0>[2.65 / 6.61 \mathrm{C} \quad 0 / 1.5 / 4]$

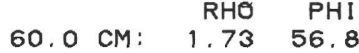
$135.0 \mathrm{CM}: \quad 1.82 \quad 51.4$

$0>[2.65 / 6.61 \mathrm{C} \quad 0 / 1.5 / 4]$ $60.0 \mathrm{CM}: \quad \begin{array}{rr}\text { RHO } & \mathrm{PHI} \\ 1.76 & 54.8\end{array}$ 35.0 CM: 1.6462 .4

$0>[2.65 / 6.61 \mathrm{C} 0 / 1.5 / 4]$

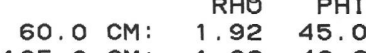
$96 \quad 42.2$

$0>[2.65 / 6.61 \mathrm{C} 0 / 1.5 / 4]$ 60.0 cM: $\quad \begin{array}{rrr}\mathrm{RHO} & \mathrm{PHI}\end{array}$ $135.0 \mathrm{CM}: 1.86 \quad 48.7$

$0>[2.65 / 6.61 \mathrm{C} \quad 0 / 1.5 / 4]$ 60.0 cM: $1.82 \quad 51.2$ $135.0 \mathrm{CM}: 1.76 \quad 54.7$

$0>[2.65 / 6.61 \mathrm{C} 0 / 1.5 / 4]$ $60.0 \mathrm{cM}: \quad \begin{array}{r}\mathrm{RHO} \\ \mathrm{PH}\end{array}$ $135.0 \mathrm{CM}: 1.7952 .8$ 


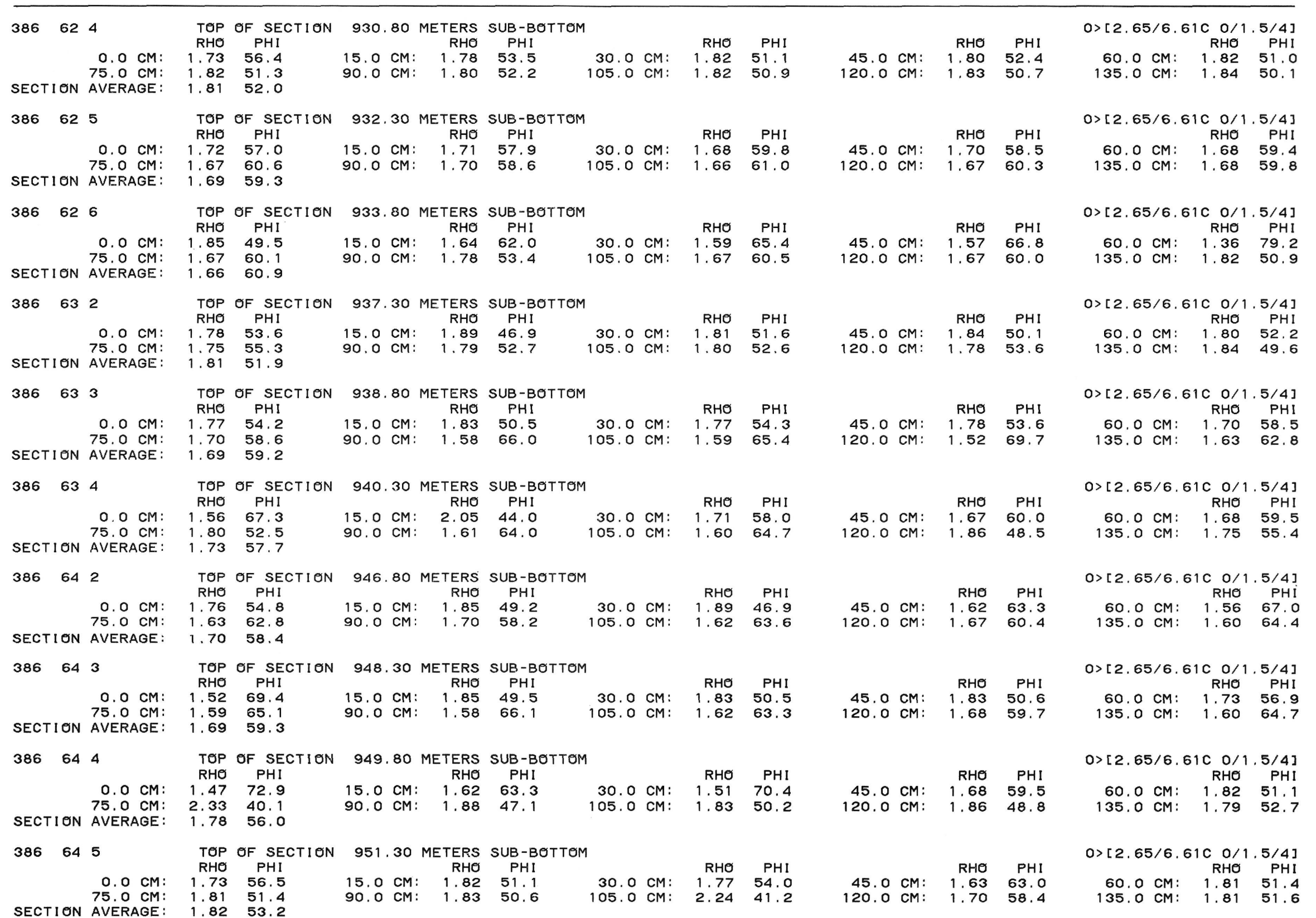


TOP OF SECTION 952.80 METERS SUB-BOTTOM

$\begin{array}{rrrrrrrrr}0.0 \mathrm{CM}: & 1.71 & \mathbf{5 7} .7 & 15.0 \mathrm{CM}: & 1.81 & 51.6 & 30.0 \mathrm{CM}: & 1.78 & \mathbf{P H} .7 \\ 75.0 \mathrm{CM}: & 1.96 & 42.7 & 90.0 \mathrm{CM}: & 2.03 & 38.0 & 105.0 \mathrm{CM}: & 1.97 & 41.6\end{array}$ SECTION AVERAGE: $\quad 1.96 \quad 42.7$

$\begin{array}{lll}386 & 65 & 1\end{array}$

\section{TOP OF SECTION 954.8O-METERS SUB-BOTTOM}

RHO PHI

15.0 $\mathrm{CM}: \quad$ RHO PHI

30.0 CM: $\quad$\begin{tabular}{rrr}
$\mathrm{RHO}$ & $\mathrm{PHI}$ \\
\hline & 1.72 & 57.2
\end{tabular}

$0.0 \mathrm{CM}: \quad 1.75 \quad 55.5$

90. $\mathrm{CM}: 2.82$ 40.2

$105.0 \mathrm{CM}: 1.91 \quad 45.8$

SECTION AVERAGE: $1.82 \quad 51.0$

$386 \quad 652$

TOP OF SECTION 956.30 METERS SUB-BOTTOM

RHO PHI

$\begin{array}{rrr}15.0 \mathrm{CM}: & \mathrm{RHO} & \mathrm{PHI} \\ & 1.54 & 68.2\end{array}$

30. $0 \mathrm{CM}:$ RHO PHI

0.0 CM: $\quad 1.67 \quad 60.1$

90.0 CM: 1.4375.

105.0 CM: $\quad 1.71 \quad 57.9$

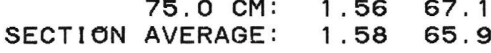

TOP OF SECTION 957.80 METERS SUB-BOTTOM

$386 \quad 653$

RHO PHI

15.0 CM: 1.77 PHI

30.0 $\mathrm{CM}: \mathrm{RHO} \mathrm{PHI}$

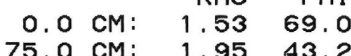

$90.0 \mathrm{CM}: 1.77 \quad 54.1$

105.0 CM: $\quad 1.73 \quad 56.4$

SECTION AVERAGE: $1.77 \quad 54.2$

$386 \quad 66 \quad 1$

TOP OF SECTION 964.20 METERS SUB-BOTTOM

$0=\left[\begin{array}{llllll}2.65 / 6.61 \mathrm{C} & 0 / 1.5 / 4] \quad 93=[2.65 / 6.02 \mathrm{C} & 0 / 1.5 / 4] & 106=[2.65 / 6.08 \mathrm{C} & 0 / 1.5 / 4]\end{array}\right.$ $31=[2.65 / 5.95 \mathrm{C} \quad 0 / 1.5 / 4] \quad 136=[2.65 / 5.95 \mathrm{C} 0 / 1.5 / 4] \quad 142=[2.65 / 6.61 \mathrm{C} 0 / 1.5 / 4]$

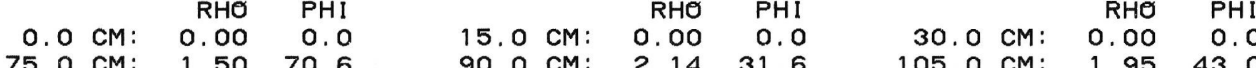

SECTION AVERAGE: $\quad 2.0437 .4$

TOP OF SECTION 965.70 METERS SUB-BOTTOM

386. $66 \quad 2$

RHO PHI RHO PHI RHO PHI

$15.0 \mathrm{CM} 2.02 \quad 38.6 \quad 30.0 \mathrm{CM}: 1.87 \quad 47.9$

SECTION AVERAGE: 1.89 47.0

$387 \quad 12$

TOP OF SECTION 33.30 METERS SUB-BOTTOM

RHO PHI

0.O CM: $1.36 \quad 79.3$

33.30 METERS SUB-BOTTOM
RHO PHI

\begin{tabular}{lll}
$75.0 \mathrm{CM}:$ & 1.44 & 74.6 \\
\hline
\end{tabular}

$\begin{array}{lll}15.0 \mathrm{CM}: & 1.42 & 75.7 \\ 90.0 \mathrm{CM}: & 1.43 & 74.9\end{array}$

30. $\mathrm{CM}$ : RHO $\mathrm{PHI}$

SECTION AVERAGE:

\section{TOP OF SECTION}

34.80 METERS SUB-BOTTOM

$\begin{array}{lll}387 & 1 & 3\end{array}$

RHO PHI

O. $\mathrm{C}$ CM: $1.40 \quad 77.2$

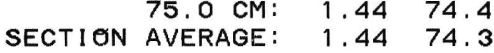

15. $\mathrm{RH}$ : $\mathrm{PHI}$

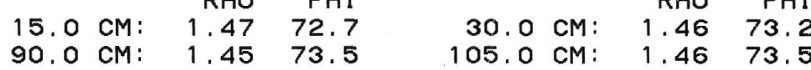

$387 \quad 14$

TOP OF SECTION 36.30 METERS SUB-BOTTOM

RHO PHI

$0.0 \mathrm{CM}: 1.44 \quad 74.8$

75.0 CM: 1.42 75.6

15.0 $\mathrm{CM}: \quad 1.45$ PHI

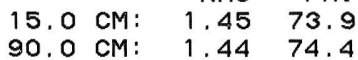

30. O $\mathrm{cm}$ RHO $\mathrm{PHI}$

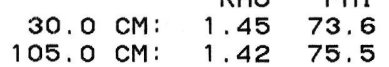

SECTION AVERAGE: $1.43 \quad 74.8$

TOP OF SECTION 37.80 METERS SUB-BOTTOM

$387 \quad 15$

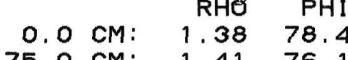

37.80 METERS SUB-BOTTOM
$15.0 \mathrm{cM}: \quad 1.40 \quad 76.9$

$\begin{array}{lll}15.0 \mathrm{CM}: & 1.40 & 76.9 \\ 90.0 \mathrm{CM}: & 1.40 & 77.1\end{array}$
$30.0 \mathrm{RHO} \quad \mathrm{PHI}$

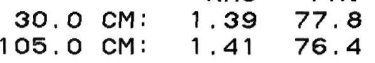

$45.0 \mathrm{~cm}: \quad \begin{array}{r}\mathrm{RHO} \\ 1.71 \quad 57.9\end{array}$ $120.0 \mathrm{cM}: \quad 2.06 \quad 36.4$ 120.0 CM: 1.8052 .1

45.0 CM: RHO PHI 120.0 CM: 1.66 60.7

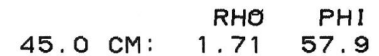
$\begin{array}{lll}120.0 \mathrm{CM}: & 1.84 & 57.9 \\ & 1.84 & 50.1\end{array}$
RHO PHI

$0>[2.65 / 6.61 \mathrm{C} 0 / 1.5 / 4]$ $60.0 \mathrm{cM}: 2.03 \quad 40.9$ 135. $\mathrm{CM}$ : $1.98 \quad 41.4$

$0>[2.65 / 6.61 \mathrm{C} 0 / 1.5 / 4]$ 60.0 CM: 1.64 62.1 135.0 CM: $1.87 \quad 47.9$

$0>[2.65 / 6.61 \mathrm{C} 0 / 1.5 / 4]$ $60.0 \mathrm{cM}: \quad \begin{array}{rr}\mathrm{RHO} & \mathrm{PHI} \\ 1.56 & 67.3\end{array}$ $135.0 \mathrm{CM}: 1.61$ 63.8

$0>[2.65 / 6.61 \mathrm{C} 0 / 1.5 / 4]$ $60.0 \mathrm{cM}: \quad \begin{array}{rr}\text { RHO } & \text { PHI } \\ 1.70 & 58.7\end{array}$ 35.0 CM: 1.7853 .6

$111=[2,65 / 6,02 \mathrm{C} 0 / 1,5 / 4]$ RHO PHI 45.0 CM: $0.00 \quad 0.0$

$118=[2,65 / 6,61 \mathrm{C} \quad 0 / 1,5 / 4]$ 60.0 cM: $\quad$ RHO PHI 135. $\mathrm{cM}: 1.00$ O.O

$0>[2.65 / 6.61 \mathrm{C} 0 / 1.5 / 4]$ 45.0 CM: RHO PHI

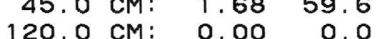
RHO PHI 35. O CM: 0.00 O. $0>[2.65 / 6.61 \mathrm{C} 0 / 1.5 / 4]$ 45. 0 cM: $\quad$ RHO PHI $120.0 \mathrm{CM}: 1.45 \quad 73.7$

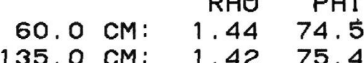

$0>[2.65 / 6.61 \mathrm{C} \quad 0 / 1.5 / 4]$ 45.0 CM: $\quad \begin{array}{r}\text { RHO } \\ \text { PHI }\end{array}$ 120.0 CM: $1.43 \quad 75.1$

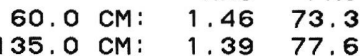

$0>[2.65 / 6.61 \mathrm{C} \quad 0 / 1.5 / 4]$

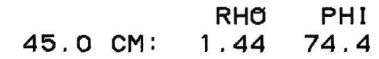
120.0 CM: $1.42 \quad 75.6$ $60.0 \mathrm{~cm}: \quad \begin{array}{rr}\mathrm{RHO} & \mathrm{PHI}\end{array}$ 135.0 CM: $1.41 \quad 76.4$

$0>[2.65 / 6.61 \mathrm{C} 0 / 1.5 / 4]$

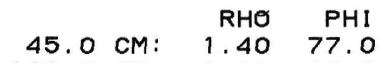
120.0 CM: $1.30 \quad 83.3$ 


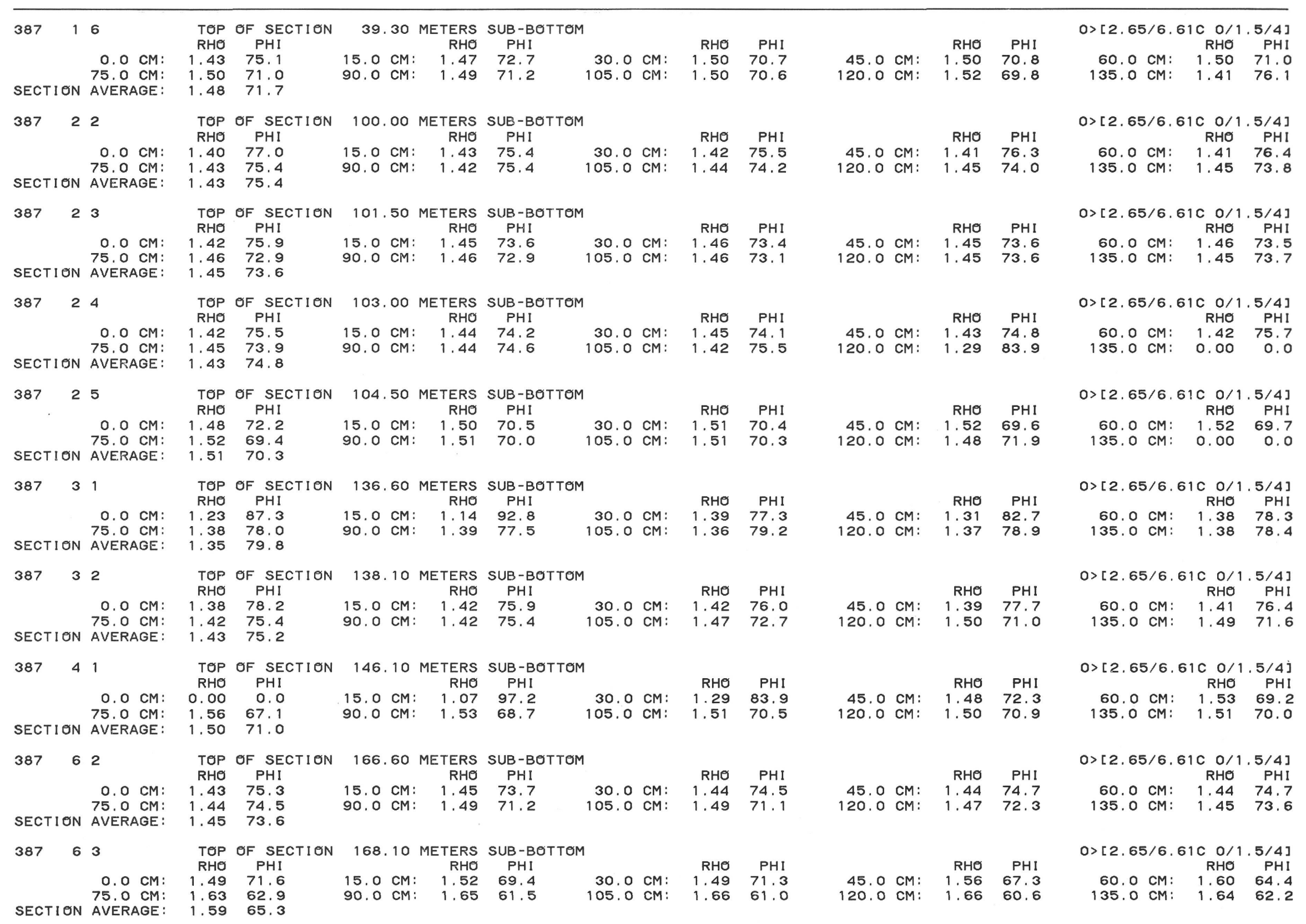


$387 \quad 7 \quad 1$

TOP OF SECTION 174.95 METERS SUB-BOTTOM

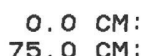

$\begin{array}{rr}\mathrm{RHO} & \mathrm{PHI} \\ 1.42 & 75.8\end{array}$

$\begin{array}{ll}1.42 & 75.8 \\ 1.40 & 77.0\end{array}$

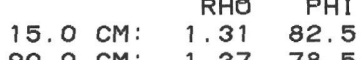

$\begin{array}{rrr}30.0 \mathrm{CM}: & 1.34 & 80.9 \\ 105.0 \mathrm{CM}: & 1.38 & 78.3\end{array}$

SECTI ON AVERAGE:

TOP OF SECTION 176.45 METERS SUB-BOTTOM

$387 \quad 72$

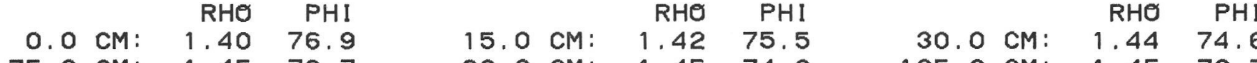

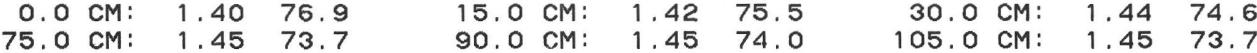

SECTION AVERAGE: 1.4474 .5

$\begin{array}{lll}387 & 7 & 3\end{array}$

TOP OF SECTION 177.95 METERS SUB-BOTTOM

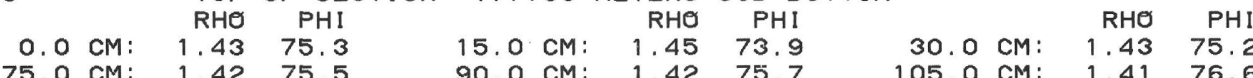

SECTION AVERAGE: $1,42 \quad 75,7$

$\begin{array}{lll}387 & 74\end{array}$

TOP OF SECTION 179.45 METERS SUB-BOTTOM

$\begin{array}{rrr}\text { 0.0 CM: } & \text { RHO } & \text { PHI } \\ 7.39 & 77.3\end{array}$

RHO PHI

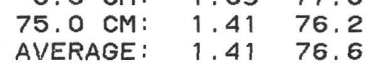

$90.0 \mathrm{CM}: \quad 1.42 \quad 75.5$

30. $0 \mathrm{CM}: \quad \mathrm{RHO} \mathrm{PHI}$

$\begin{array}{lll}105.0 \mathrm{CM}: & 1.42 \quad 75.7\end{array}$

SECTION AVERAGE:

$387 \quad 75$

TOP OF SECTION 180.95 METERS SUB-BOTTOM

$0.0 \mathrm{CM}$
$75.0 \mathrm{CM}$

RHO $\mathrm{PH}$

75.0 CM: 1.39 77.3

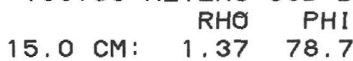

90.0 CM: 1.39 77.3

30. $\mathrm{CM}: \mathrm{RHO} \mathrm{PHI}$

30.0 CM: $\quad 1.36 \quad 79.5$

.3878 .3

TOP OF SECTION 182.45 METERS SUE-BOTTOM

$387 \quad 76$

$\begin{array}{rr}\text { RHO } & \text { PHI } \\ 1.35 & 79.9\end{array}$

$15.0 \mathrm{CM}: \quad 1.39 \quad 77.3$

30. $\mathrm{CM}: \mathrm{RHO} \mathrm{PHI}$

$0.0 \mathrm{CM}$

SECTION AVERAGE:

$\begin{array}{ll}1.41 & 76.6 \\ 1.38 & 78.1\end{array}$

15.0 CM: $1.41,76.6$

$\begin{array}{lll} & \\ 0 & & \\ \text { CM: } & 1.39 & 77.5 \\ & & \end{array}$

$\begin{array}{lll}387 & 8 & 1\end{array}$

TOP OF SECTION 184.20 METERS SUB-BOTTOM
RHO PHI

$0.0 \mathrm{CM}:$

O. 0 15.0 CM: 0.00 RHO PHI

$\begin{array}{lllllll}1.35 & 79.8 & & & & & \end{array}$

SECTION AVERAGE: $\quad 1.37 \quad 78,9$

$387 \quad 82$

TOP OF SECTION 185.70 METERS SUB-BOTTOM

RHO PHI RHO PHI RHO PHI

$0.0 \mathrm{CM}: \quad 1.3878 .0$

$\begin{array}{rrr}\text { OT.0 CM: } & 1.38 & 78.0 \\ \text { AVERAGE: } & 1.38 & 78.0 \\ \text { AVERAG } & 1.39 & 77.4\end{array}$

15.0 CM: $\quad 1.42 \quad 75.9$

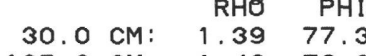

$90.0 \mathrm{CM}: \quad 1.38 \quad 78.4$

$105.0 \mathrm{CM}: 1.4076 .9$

SECTION AVERAGE:

TOP OF SECTION 194.10 METERS SUB-BOTTOM

. $\mathrm{CM}: \quad 1$ RO $\mathrm{PHI}$

CM: $\quad 1.39$ 77.5

SECTION AVERAGE: $1.42 \quad 75.7$

15.0 CM: $1.41 \quad 76.4$

$30.0 \mathrm{CM}:$ RHO $\mathrm{PHI}$

$\begin{array}{lll}105.0 \mathrm{CM}: & 1.45 & 73.7\end{array}$

38792

TOP OF SECTION 195.60 METERS SUB-BOTTOM

$\begin{array}{rrr}0.0 \mathrm{CM}: & 1.48 & \mathrm{PHI} \\ 75.0 \mathrm{CM}: & 1.45 & 73.7\end{array}$

SECTION AVERAGE: $1.47 \quad 72.7$ $\begin{array}{llllll}15.0 & \mathrm{CM}: & \mathrm{RHO} & \mathrm{PHI} & \mathrm{RHO} & \mathrm{PHI} \\ 1.49 & 71.2 & 30.0 \mathrm{~cm}: & 1.46 & 73.4\end{array}$

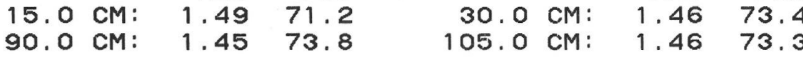

45. $\mathrm{CM}: \quad \mathrm{RHO} \quad \mathrm{PHI}$ 120.0 CM: 1.4574.

RHO PHI 45.0 CM: $1.45 \quad 74.1$

RHO PHI

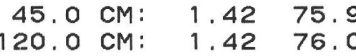

$45.0 \mathrm{cM}: \quad \begin{array}{r}\mathrm{RHO} \\ \mathrm{PHI}\end{array}$

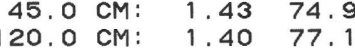

RHO $\mathrm{PHI}$ $\begin{array}{rrr}45.0 \mathrm{CM}: & 1.38 & 78.4 \\ 120.0 \mathrm{CM}: & 1.42 & 75.7\end{array}$

45. PHI

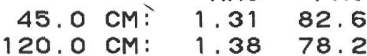

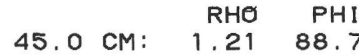
120.0 CM: 1.4088 .7

45. $\mathrm{CM}: \quad \mathrm{RHO} \quad \mathrm{PHI}$ 120.0 CM: $\quad 1.38$ 78.3

45. O CM: $\quad \begin{array}{r}\mathrm{RHO} \\ \mathrm{PHI}\end{array}$ 120.0 CM: 1.46 73.3

45. CM: RHO PHI 45. $\mathrm{CM}: 1.46 \quad 73.4$
$0>[2,65 / 6,61 \mathrm{C} 0 / 1,5 / 4]$ $60.0 \mathrm{cM}: \quad \begin{array}{r}\mathrm{RHO} \\ 1.41\end{array}$ 135. $\mathrm{CM}: 1.4275 .6$

$0>[2.65 / 6.61 \mathrm{C} \quad 0 / 1.5 / 4]$ 60. $\mathrm{cM}: \quad \mathrm{RHO} \mathrm{PH}$ 135. O CM: $1.43 \quad 75.3$

$0>[2.65 / 6.61 \mathrm{C} 0 / 1,5 / 4]$ $60.0 \mathrm{cM}: 1.4176 .3$ 135.0 CM: 1.4077.

$0>[2.65 / 6,61 \mathrm{C} 0 / 1,5 / 4]$ 60.0 CM: 1.42 PHO 135.0 CM: 1.38 78.3

$0>[2.65 / 6.61 \mathrm{C} \quad 0 / 1.5 / 4]$ $60.0 \mathrm{~cm}: \quad \begin{array}{rr}\mathrm{RHO} & \mathrm{PH} \\ 1.37 & 78.5\end{array}$ $135.0 \mathrm{CM}: 0.00 \quad 0.0$

$0>[2.65 / 6.61 \mathrm{C} \quad 0 / 1.5 / 4]$ 60.0 CM: $1.40 \quad 77.1$

$0>[2,65 / 6,61 \mathrm{C} 0 / 1.5 / 4]$ RHO PHI $135.0 \mathrm{cM}: 1.4275 .8$

$0>[2.65 / 6.61 \mathrm{C} 0 / 1.5 / 4]$ $\begin{array}{rrr}60.0 \mathrm{CM}: & 1.39 & 77.4 \\ 135.0 \mathrm{CM}: & 1.40 & 77.1\end{array}$

$0>[2.65 / 6.61 \mathrm{C} 0 / 1.5 / 4]$ 60.0 CM: $1.38 \quad 78.4$ $135.0 \mathrm{cM}: 1.4573 .5$

$0>[2.65 / 6.61 \mathrm{C} 0 / 1.5 / 4]$ $60.0 \mathrm{~cm}: \quad \begin{array}{rr}\mathrm{RHO} & \mathrm{PHI} \\ 1.46 & 73.2\end{array}$ $135.0 \mathrm{CM}: 1.5170 .1$ 
$387 \quad 9$

RHO PHI RHO PHI

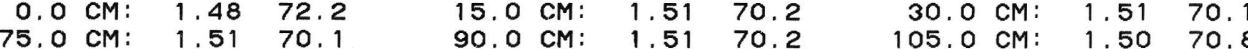

SECTION AVERAGE: 1.5170 .2

38794

TOP OF SECTION 198.60 METERS SUB-BOTTOM

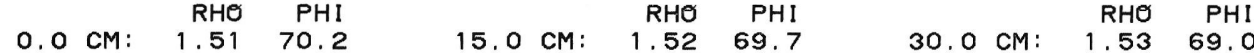

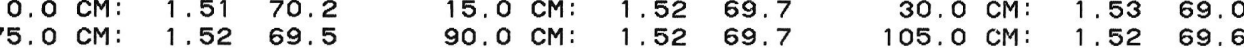

SECTION AVERAGE: 1.5269 .5

$387 \quad 95$

TOP OF SECTION 200.10 METERS SUB-BOTTOM

$0.0 \mathrm{cM}$ : RHO PHI RHO PHI RHO PHI $\begin{array}{rrrrrrrrr}0.0 \mathrm{CM}: & 1.48 & 71.7 & 15.0 \mathrm{CM}: & 1.51 & 69.9 & 30.0 \mathrm{CM}: & 1.51 & 69.9 \\ 75.0 \mathrm{CM}: & 1.52 & 69.8 & 90.0 \mathrm{CM}: & 1.52 & 69.3 & 105.0 \mathrm{CM}: & 1.51 & 70.2\end{array}$

SECTION AVERAGE: $\quad 1.5169 .8$

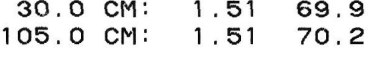

38796

TOP OF SECTION 201.60 METERS SUB-BOTTOM

0.0 CM: $\quad 1.43 \quad 75.4$

15. $\mathrm{CM}$ : RHO PHI

$\begin{array}{rrr}30.0 \mathrm{CM}: & \text { RHO } & \mathrm{PHI} \\ 105.0 \mathrm{CM}: & 1.45 & 71.5 \\ 74.1\end{array}$

90.0 CM: 1.4275 .7

SECTION AVERAGE: $\quad 1.46 \quad 73.4$

$387 \quad 10 \quad 1$

TOP OF SECTION 203.60 METERS SUB-BOTTOM

$$
\begin{array}{rrr}
0.0 \mathrm{CM}: & \begin{array}{r}
\mathrm{RHO} \\
\mathrm{PHI}
\end{array} \quad \mathrm{PH} \\
79.0
\end{array}
$$

75.0 CM: $\quad 1.3779 .0$

SECTION AVERAGE: $1.42 \quad 75.9$

\begin{abstract}
$\begin{array}{rrr}15.0 \mathrm{CM}: & \text { RHO } & \mathrm{PHI} \\ 1.38 & 78.1\end{array}$
\end{abstract}
$30.0 \mathrm{CM}: \quad \begin{array}{rrr}\mathrm{RHO} & \mathrm{PHI} \\ 1.44 & 74.6\end{array}$

$90.0 \mathrm{CM}: 1.4275 .8$

$\begin{array}{lll}387 & 102\end{array}$

$\begin{array}{rrr}0.0 \mathrm{CM}: & \begin{array}{r}\mathrm{RHO} \\ \mathrm{PH}\end{array} \quad \mathrm{PH} \\ 7.45 & 73.9\end{array}$

75. O CM: $\quad 1.43 \quad 75.1$

15.0 CM: $1.53 \quad \mathrm{PHI}$

90.0 CM: $1.44 \quad 74.6$

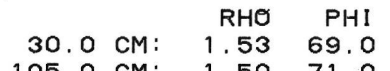

SECTION AVERAGE:

TOP OF SECT

TOP OF SECTION 206.60 METERS SUB-BOTTOM

$387 \quad 103$

$\begin{array}{rrr}0.0 \mathrm{CM}: & \text { RHO } & \text { PHI } \\ 75.0 \mathrm{CM}: & 1.48 & 71.8 \\ & 1.43 .0\end{array}$

SECTION AVERAGE: $\begin{array}{lll}1.47 & 72.7\end{array}$

$\begin{array}{rrr}15.0 \mathrm{cM}: & \mathrm{RHO} & \mathrm{PHI} \\ & 1.46 & 73.2\end{array}$

$\begin{array}{lll}15.0 \mathrm{CM}: & 1.46 & 73.2 \\ 90.0 \mathrm{CM}: & 1.41 & 76.1\end{array}$

30.0 cM: $\quad$ RHO PHI

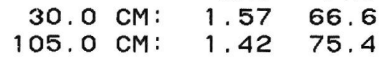

$387 \quad 104$

TOP OF SECTION 208.10 METERS SUB-BOTTOM

RHO PHI

O.O CM: $\quad 1.40 \quad 77.2$

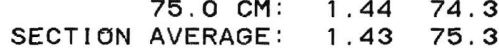

$\begin{array}{rrr}15.0 \mathrm{CM}: & \mathrm{RHO} & \mathrm{PHI} \\ & 1.41 & 76.2\end{array}$

30.0 CM: $\quad$\begin{tabular}{rrr} 
RHO & PHI \\
\hline & 1.44 & 74.7
\end{tabular}

90.0 CM: $1.5269 .5 \quad 105.0 \mathrm{cM}: 1.44 \quad 74.5$

$387 \quad 105$

TOP OF SECTION 209.60 METERS SUB-BOTTOM

RHO PHI

\begin{tabular}{rrr}
$0.0 \mathrm{CM}:$ & RHO & PHI \\
$75.0 \mathrm{CM}:$ & 1.47 & 77.9 \\
\hline & 72.7
\end{tabular}

$\begin{array}{rrr}15.0 \mathrm{CM}: & \text { RHO } & \text { PHI } \\ & 1.39 & 77.8\end{array}$

30. 0 CM: $\begin{array}{rr}\text { RHO } & \text { PHI } \\ 1.44 & 74.4\end{array}$

90.0 CM: $\quad 1.53$ 69.2

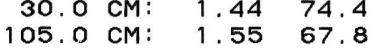

SECTION AVERAGE: $1.47 \quad 72.9$

TOP OF SECTION 211.10 METERS SUB-BOTTOM

$387 \quad 106$

$\begin{array}{rrr}0.0 \mathrm{CM}: & \text { RHO } & \mathrm{PHI} \\ 75.0 \mathrm{CM}: & 1.38 & 74.9 \\ 78.4 & 78.4\end{array}$

$\begin{array}{rrr}15.0 \mathrm{CM}: & 1.42 & 75.8 \\ 90.0 \mathrm{CM}: & 1.34 & \mathbf{8 0 . 4}\end{array}$

30.0 CM: $\begin{array}{rrr}\text { RHO } & \mathrm{PHI} \\ 1.38 & 78.1\end{array}$

SECTION AVERAGE: 1.4176 .2
45. O CM: RHO PHI

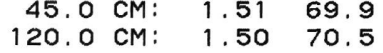

45. O CM: $\quad \begin{array}{rr}\text { RHO } & \mathrm{PHI} \\ 1.52 & 69.5\end{array}$

$120.0 \mathrm{CM}: 1.5468 .5$

45. $\mathrm{CM}$ : $1.5 \mathrm{PHI}$

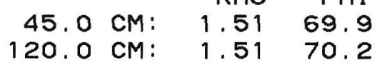

45. O CM: $\begin{array}{rr}\text { RHO } & \text { PHI } \\ 1.50 & 71.0\end{array}$ $\begin{array}{rrr}45.0 \mathrm{CM}: & 1.50 & 71.0 \\ 120.0 \mathrm{CM}: & 1.46 & 73.2\end{array}$

$\begin{array}{rrr}45.0 \mathrm{CM}: & \begin{array}{r}\mathrm{RHO} \\ \text { PHI }\end{array} & 74.6 \\ 120.0 \mathrm{CM}: & 1.39 & 77.6\end{array}$

45.0 CM: $\begin{array}{rr}\text { RHO } & \mathrm{PHI} \\ 1.53 & 68.6\end{array}$

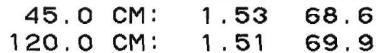

45. O CM: $\quad \begin{array}{rr}\mathrm{RHO} & \mathrm{PHI} \\ 1.55 & 67.5\end{array}$ $\begin{array}{rll}120.0 \mathrm{CM}: & 1.38 & 78.3\end{array}$

45. O CM: $\begin{array}{rr}\mathrm{RHO} & \mathrm{PHI} \\ 1.43 & 74.9\end{array}$ 120.0 CM: 1.3281 .6

45. O CM: $\begin{array}{rr}\text { RHO } & \text { PHI } \\ 1.45 & 73.9\end{array}$

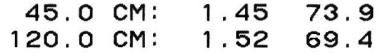

$\begin{array}{rrr}\text { 45. O CM: } & \text { RHO } & \text { PHI } \\ 1.42 & 75.7\end{array}$
$0>[2.65 / 6.61 \mathrm{C} 0 / 1.5 / 4]$ 60.0 CM: 1.51 PO.3 $135.0 \mathrm{cM}: 1.5667 .0$

$0>[2.65 / 6.61 \mathrm{C} \quad 0 / 1.5 / 4]$ 60.0 CM: 1.52 69.3 $135.0 \mathrm{cM}: 1.5070 .5$

$0>[2.65 / 6.61 \mathrm{C} 0 / 1.5 / 4]$ $60.0 \mathrm{cM}: \quad \begin{array}{rr}\mathrm{RHO} & \mathrm{PHI} \\ 1.50 & 70.8\end{array}$ 135.0 CM: 1.53 69.1

$0>[2.65 / 6.61 \mathrm{C} 0 / 1.5 / 4]$

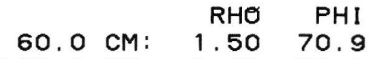
$35.0 \mathrm{CM}: 1.4673 .0$

$0>[2.65 / 6.61 \mathrm{C} \quad 0 / 1.5 / 4]$

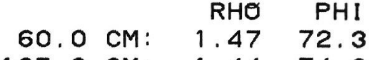

$0>[2.65 / 6.61 \mathrm{C} 0 / 1,5 / 4]$

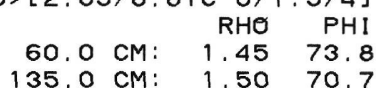

$0>[2.65 / 6.61 \mathrm{C} \quad 0 / 1.5 / 4]$

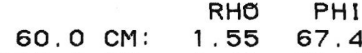
$135.0 \mathrm{CM}: 1.3878 .1$

$0>[2.65 / 6.61 \mathrm{C} 0 / 1.5 / 4]$

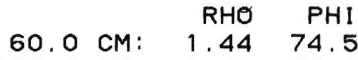
$135.0 \mathrm{cM}: 1.41 \quad 76.1$

$0>[2.65 / 6.61 \mathrm{C} \quad 0 / 1.5 / 4]$ $60.0 \mathrm{~cm}: \quad \begin{array}{rr}\mathrm{RHO} & \mathrm{PHI}\end{array}$ $135.0 \mathrm{~cm}: 1.4474 .3$

$0>[2.65 / 6.61 \mathrm{C} \quad 0 / 1.5 / 4]$ $\begin{array}{rrr}60.0 \mathrm{cM}: & \text { RHO } & \text { PHI } \\ 135.0 \mathrm{cM}: & 1.43 & 76.3\end{array}$ 
$387 \quad 142$

TOP OF SECTION 242.80 METERS SUB-BOTTOM

\section{$0.0 \mathrm{CM}$ :}

RHO PHI

$75.0 \mathrm{CM}:$

SECTIOON AVERAGE

$\begin{array}{lll}387 & 16 & 1\end{array}$

$\begin{array}{ll}1.70 & 58.2 \\ 1.65 & 61.3\end{array}$

15.0 cM: $\begin{array}{rrr}\text { RHO } & \text { PHI } \\ 1.57 & 66.7\end{array}$

$\begin{array}{lll}15.0 \mathrm{CM}: & 1.57 & 66.7 \\ 90.0 \mathrm{CM}: & 1.69 & 59.3\end{array}$

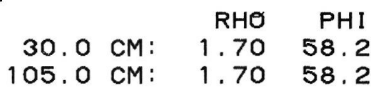

TOP OF SECTION 260.10 METERS SUB-BOTTOM

\begin{tabular}{llllllll} 
RHO & PHI & \multicolumn{1}{c}{ RHO } & PHI \\
0.00 & 0.0 & $15.0 \mathrm{cM}:$ & 0.00 & 0.0 & $30.0 \mathrm{cM}:$ & 0.00 & 0.0
\end{tabular}

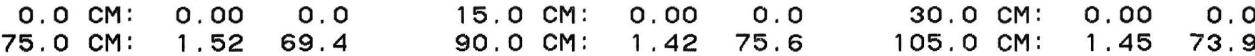
SECTION AVERAGE: 1.46 73.1

$387 \quad 162$

TOP OF SECTION 261.60 METERS SUB-BOTTOM

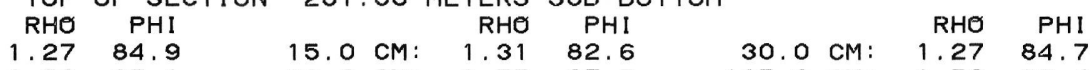

$\begin{array}{rlllllrll}0.0 \mathrm{CM}: & 1.27 & 84.9 & 15.0 \mathrm{CM}: & 1.31 & 82.6 & 30.0 \mathrm{CM}: & 1.27 & 84.7 \\ 75.0 \mathrm{CM}: & 1.56 & 67.1 & 90.0 \mathrm{CM}: & 1.55 & 67.5 & 105.0 \mathrm{CM}: & 1.56 & 67.4\end{array}$

SECTION AVERAGE: 1.4573 .9

TOP OF SECTION 263.10 METERS SUB-BOTTOM

$387 \quad 163$

$0.0 \mathrm{CM}: \quad \begin{aligned} & \mathrm{RHO} \\ & \mathrm{PHI}\end{aligned}$

$\begin{array}{rrrrrrr} & & & \end{array}$

SECTION AVERAGE: 1.53 69.0

$387 \quad 17 \quad 1$

TOP OF SECTION 269.60 METERS SUB-BOTTOM

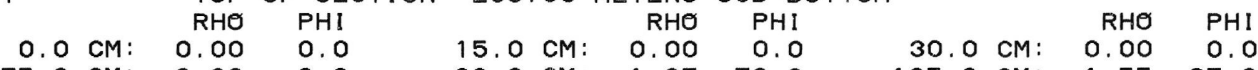

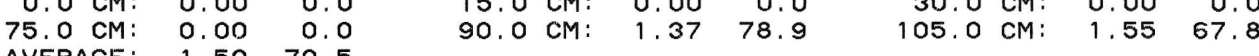

SECTION AVERAGE: 1.5070 .5

$387 \quad 172$

TOP OF SECTION 271.10 METERS SUB-BOTTOM

RHO PHI RHO PHI

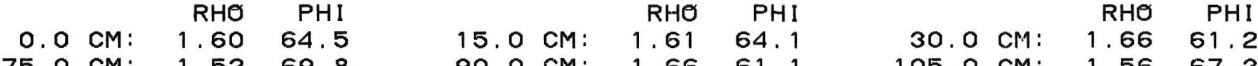

SECTION AVERAGE: 1.5766 .2

TOP OF SECTION 288.70 METERS SUB-BOTTOM

$\begin{array}{lll}387 & 18 & 1\end{array}$

$0.0 \mathrm{CM}:$

$.38 \quad 77.9$

$15.0 \mathrm{cM}: \quad 1.36 \quad 79.4$

9.4
69.2

30.0 CM: $\quad$ RHO PHI

$105.0 \mathrm{CM}: 1.55 \quad 67.8$

SECTI ON AVERAGE

TOP OF SECTION 307.70 METERS SUB-BOTTOM

$\begin{array}{lll}387 & 19 & 1\end{array}$

$0.0 \mathrm{CM}$ :

$\mathrm{RHO} \mathrm{PHI}$

$75.0 \mathrm{CM}:$

$\begin{array}{rr}.00 & 0.0 \\ 1.40 & 76.7\end{array}$

$15.0 \mathrm{cM}: \quad$ RHO PHI

$30.0 \mathrm{RHO} \quad \mathrm{PHI}$

$.40 \quad 76.7$

$90.0 \mathrm{CM}: \quad 1.48 \quad 72.3$

SECTI ON AVERAGE:

TOP OF SECTION 309.20 METERS SUB-BOTTOM

$\begin{array}{lll}387 & 192\end{array}$

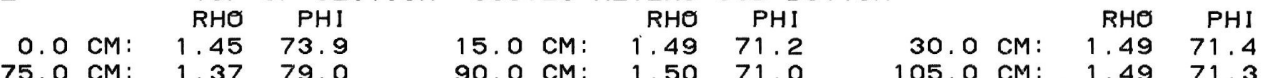

SECTION AVERAGE: 1.4871 .9

TOP OF SECTION 310.70 METERS SUB-BOTTOM

$\begin{array}{lll}387 & 193\end{array}$

RHO PHI

O. $\mathrm{CM}: \quad 1.5965 .0$

15.0 CM: $\quad 1.60$ RHO 64.4

30.0 CM: $\quad \begin{array}{rrr}\mathrm{RHO} & \mathrm{PHI} \\ & 1.63 & 62.8\end{array}$

90.0 CM: 1.56 67.0

$105.0 \mathrm{CM}: \quad 1.55 \quad 67.7$

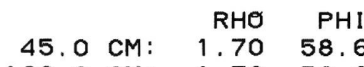

$\begin{array}{rrr}45.0 \text { CM: } & 1.70 & 58.6 \\ 120.0 \text { CM: } & 1.73 & 56.8\end{array}$

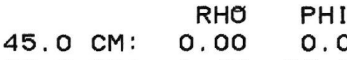

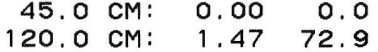

45. O CM: $\quad \begin{array}{r}\text { RHO } \\ \text { PHI }\end{array}$

$120.0 \mathrm{CM}: \quad 0.00 \mathrm{74.6}$

45.0 CM: $\begin{array}{rr}\text { RHO } & \text { PHI } \\ 1.55 & 67.7\end{array}$

$120.0 \mathrm{CM}: 1.4573 .8$

45. O cM: $\begin{array}{lll}\text { RHO } & \text { PHI } \\ 0.00 & 0.0\end{array}$

120.0 CM: $\begin{array}{lr}1.51 & 70.4\end{array}$

45. $\mathrm{cM}: \quad \mathrm{RHO} \quad \mathrm{PHI}$

$\begin{array}{rrr}45.0 \mathrm{CM}: & 1.49 & 71.6 \\ 120.0 \mathrm{CM}: & 1.53 & 69.2\end{array}$

45. O CM: $\begin{array}{rr}\text { RHO } & \text { PHI } \\ 1.51 & 70.0\end{array}$

$\begin{array}{rrr}45.0 \mathrm{CM}: & 1.51 & 70.0 \\ 120.0 \mathrm{CM}: & 1.53 & 69.1\end{array}$

45. O CM: $\quad \begin{array}{r}\text { RHO } \\ \mathrm{PHI}\end{array}$

45. O CM: $1.42 \quad 75.8$

RHO PHI

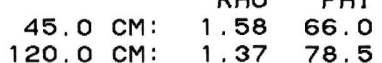

45. CM: $\begin{array}{rr}\mathrm{RHO} & \mathrm{PHI} \\ 1.63 & 62.8\end{array}$

$\begin{array}{rll}45.0 \mathrm{CM}: & 1.63 & 62.8 \\ 120.0 \mathrm{CM}: & 1.63 & 62.5\end{array}$
$0>[2.65 / 6,61 \mathrm{C} 0 / 1.5 / 4]$

$60.0 \mathrm{cM}: \quad \begin{array}{r}\mathrm{RHO} \\ 1.66\end{array}$

$135.0 \mathrm{cM}: \quad 1.60 \quad 64.5$

$0>[2.65 / 6.61 \mathrm{C} 0 / 1.5 / 4]$ $60.0 \mathrm{cM}: \quad \begin{array}{rr}\mathrm{RHO} & \mathrm{PHI} \\ 1.45 & 73.6\end{array}$ 135.0 CM: 1.4374 .9

$0>[2.65 / 6.61 \mathrm{C} 0 / 1.5 / 4]$ $\begin{array}{rrr}60.0 \mathrm{CM}: & \text { RHO } & \mathrm{PHI} \\ & 1.60 & 64.7\end{array}$ $0>[2.65 / 6.61 \mathrm{C} 0 / 1.5 / 4]$ $60.0 \mathrm{cM}: \quad \begin{array}{r}\mathrm{RHO} \\ \mathrm{PHI}\end{array}$ 135.0 CM: 1.3083 .2

$0>[2.65 / 6.61 \mathrm{C} 0 / 1.5 / 4]$ $60.0 \mathrm{cM}: \quad 0.00 \quad 0.0$ 135.0 CM: $1.56 \quad 67.2$

$0>[2.65 / 6.61 \mathrm{C} \quad 0 / 1.5 / 4]$

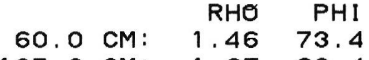
$135.0 \mathrm{CM}: 1.6760 .4$

$0>[2.65 / 6.61 \mathrm{C} \quad 0 / 1.5 / 4]$ 60.0 CM: 1.3182 .4 $0>[2.65 / 6.61 \mathrm{C} 0 / 1.5 / 4]$

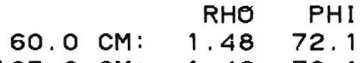
135.0 CM: 1.4673 .1

$0>[2.65 / 6.61 \mathrm{C} \quad 0 / 1.5 / 4]$ $60.0 \mathrm{cM}: \quad \mathrm{RHO} \quad \mathrm{PHI}$ 135.0 CM: $1.42 \quad 75.4$ $0>[2.65 / 6.61 \mathrm{C} \quad 0 / 1.5 / 4]$ $60.0 \mathrm{~cm}: \quad \mathrm{RHO} \quad \mathrm{PHI}$ $135.0 \mathrm{cM}: 1.3977 .6$ SECTION AVERAGE: 1.5965 .4 


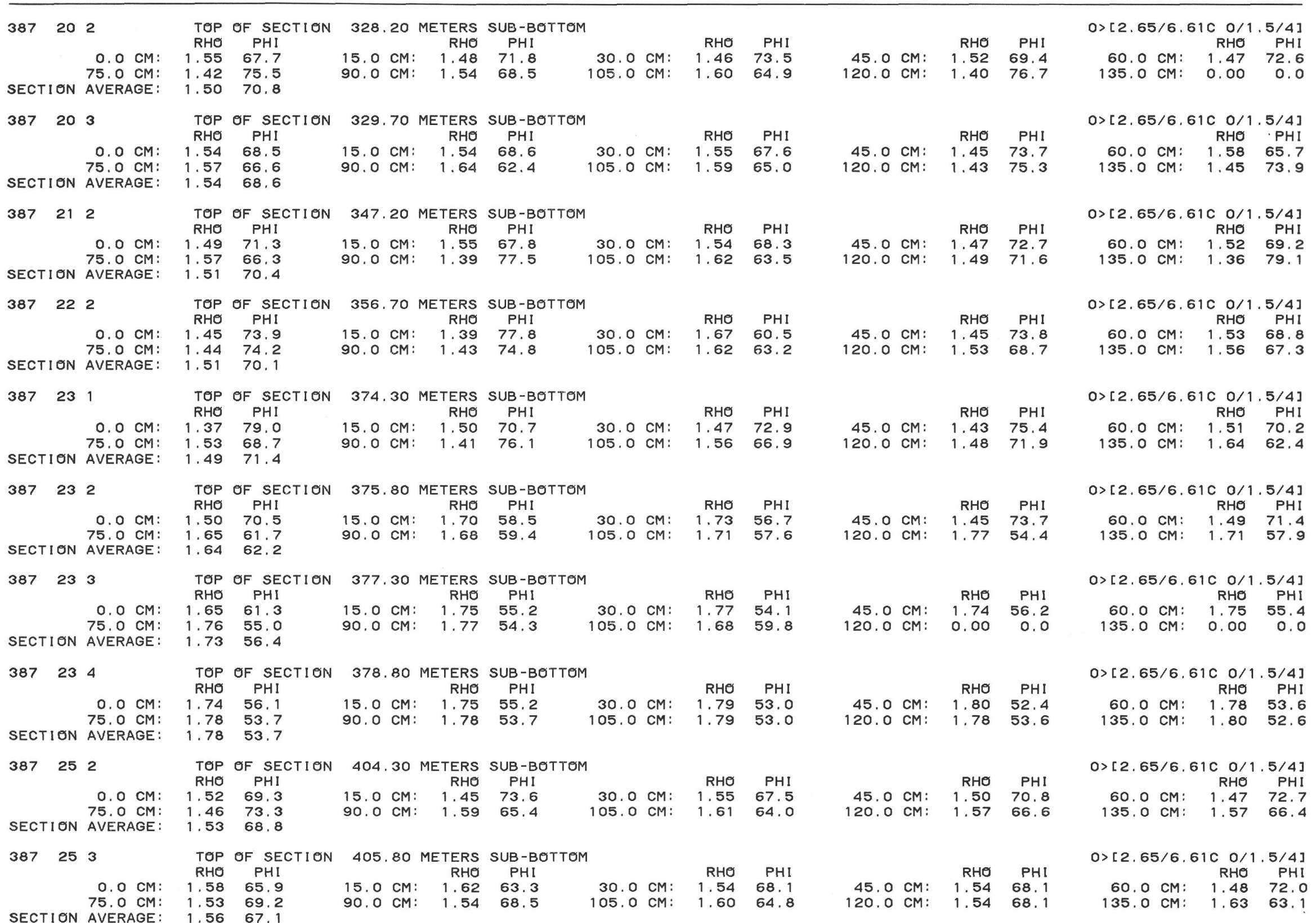


387261

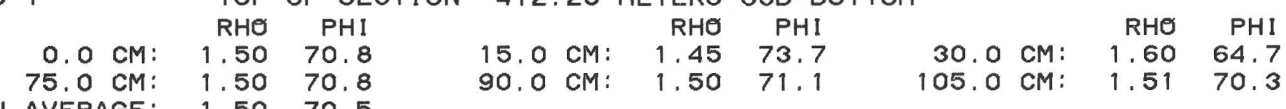
SECTION AVERAGE: 1.5070 .5

387262 $\begin{array}{rrr}0.0 \mathrm{CM}: & \mathrm{RHO} & \mathrm{PHI} \\ 75.50 & 70.6\end{array}$ $\begin{array}{lll}75.0 \mathrm{CM}: & 1.57 & 66.6 \\ \text { SECTION AVERAGE: } & 1.55 & 67.7\end{array}$

387263

$$
\text { TOP OF SECTION }
$$

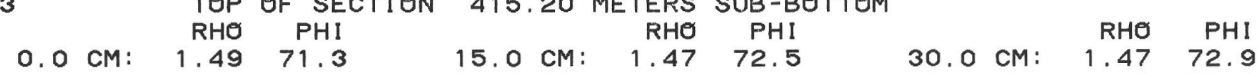

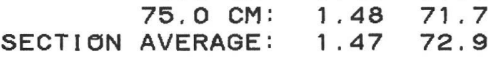

$38727 \quad 1$

\section{TOP OF SECTION}

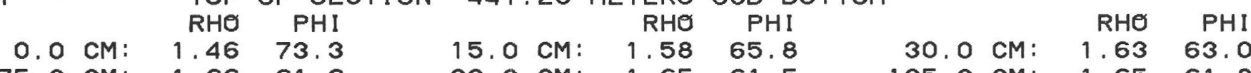
SECTION AVERAGE: 1.5965 .0

$\begin{array}{lll}387 & 27 \quad 2\end{array}$

TOP OF SECTION 442.75 METERS SUB-BOTTOM

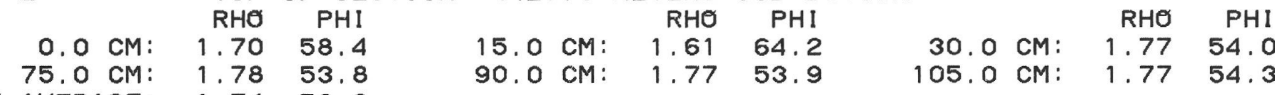
SECTION AVERAGE: $1.74 \quad 56.0$

$\begin{array}{lll}387 & 27 & 3\end{array}$

TøP OF SECTION 444.25 METERS SUB-BOTTOM

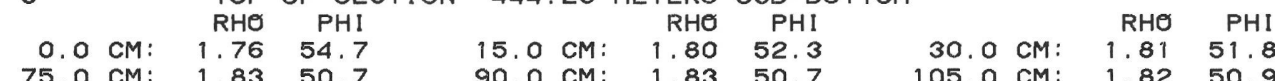
SECTION AVERAGE: 1.8151 .6

$\begin{array}{lll}387 & 27 & 4\end{array}$

\section{TOP OF SECTION 445.75 METERS SUB-BOTTOM}

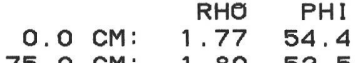

445.75 METERS SUB-BOTTOM
RHO PHI

$\begin{array}{lll}\text { 75. O CM: } & 1.80 & \mathbf{5 2 . 5} \\ \text { AVERAGE: } & 1.79 & \mathbf{5 2 . 7}\end{array}$

SECTION AVERAGE

$387 \quad 275$

\section{TOP OF SECTION 447.25 METERS SUB-BOTTOM}

RHO PHI

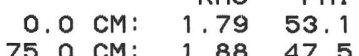

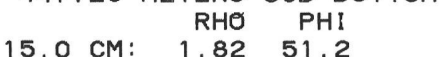

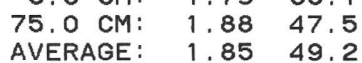

90.0 CM: 1.9145 .7

30. $0 \mathrm{CM}: \quad 1.82 \quad 51.2$

05.0 CM: $1.93 \quad 44.6$

SECTION AVERAGE

$387 \quad 276$

TOP OF SECTION 448.75 METERS SUB-BOTTOM

$\begin{array}{rrr}0.0 \mathrm{CM}: & \mathrm{RHO} & \mathrm{PH} \\ 75.0 \mathrm{CM}: & 1.98 & 50.5 \\ \text { 75 } & 41.5 \\ \text { AVERAGE: } & 1.96 & 42.8\end{array}$

RHO PHI RHO PHI

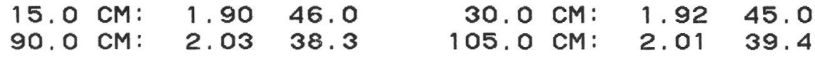
SECTION AVERAGE:

$387 \quad 292$

TOP OF SECTION 470.90 METERS SUB-BOTTOM

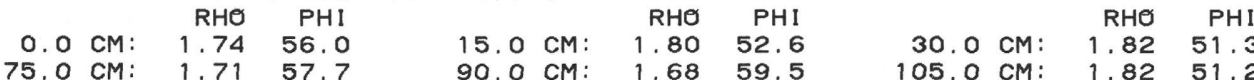
SECTION AVERAGE: $1.76 \quad 54.8$
45. 0 CM: $\quad \begin{array}{rr}\mathrm{RHO} & \mathrm{PHI} \\ 1.51 & 70.2\end{array}$ 120.0 CM: $1.48 \quad 72.3$

45. $\mathrm{CM}: \begin{array}{rr}\mathrm{RHO} & \mathrm{PHI} \\ 1.53 & 68.7\end{array}$ 120.0 CM: $0.00 \quad 0.0$

45. O CM: $\quad$\begin{tabular}{rrr}
$\mathrm{RHO}$ & $\mathrm{PHI}$ \\
\hline & 69.7
\end{tabular} $\begin{array}{rrr}45.0 \mathrm{CM}: & 1.52 & 69.7 \\ 120.0 \mathrm{CM}: & 1.41 & 76.4\end{array}$

45. $\mathrm{RHO} \quad \mathrm{PHI}$

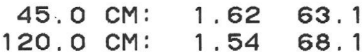

$45.0 \mathrm{RHO} \mathrm{PHI}$ 45. O CM: 1.78 53.5 RHO PHI

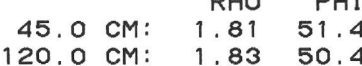

45. $\mathrm{cm}: \quad \mathrm{RHO} \quad \mathrm{PHI}$ 120.0 CM: 1.8052 .0

45. 0 CM: $\quad \begin{array}{rr}\text { RHO } & \text { PHI }\end{array}$ $\begin{array}{rrr}45.0 \mathrm{CM}: & 1.82 & 51.2 \\ 120.0 \mathrm{CM}: & 1.88 & 47.3\end{array}$

15. $\mathrm{RM}$ : $\mathrm{RHO} \mathrm{PHI}$ $\begin{array}{rrr}45.0 \mathrm{CM}: & 1.91 & 45.5 \\ 120.0 \mathrm{CM}: & 2.05 & 36.9\end{array}$

RHO PHI 45. O CM: 1.83 50.6
$0>[2.65 / 6.61 \mathrm{C} \quad 0 / 1.5 / 4]$ 60.0 CM: $\quad \begin{array}{r}\mathrm{RHO} \\ 1.54\end{array}$ $135.0 \mathrm{CM}: 1.3480 .7$

$0>[2.65 / 6.61 \mathrm{C} \quad 0 / 1.5 / 4]$ 60.0 CM: 1.54 RH. $135.0 \mathrm{CM}: \quad 0.00 \quad 0.0$

$0>[2.65 / 6.61 \mathrm{C} 0 / 1.5 / 4]$ $60.0 \mathrm{cM}: \quad \begin{array}{r}\mathrm{RHO} \\ \mathrm{PHI}\end{array}$ $135.0 \mathrm{cM}: 1.46 \quad 73.1$

$0>[2.65 / 6.61 \mathrm{C} \quad 0 / 1.5 / 4]$

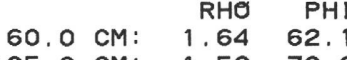

$0>[2.65 / 6,61 \mathrm{C} 0 / 1.5 / 4]$ $\begin{array}{lrr} & \text { RHO } & \text { PHI } \\ 60.0 \mathrm{cM}: & 1.73 & 56.7\end{array}$

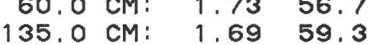

$0>[2.65 / 6.61 \mathrm{C} 0 / 1.5 / 4]$ $\begin{array}{rrrr}60.0 & \mathrm{CM}: & 1.81 & 51.5 \\ 135.0 \mathrm{CM}: & 1.81 & 51.8\end{array}$

$0>[2.65 / 6.61 \mathrm{C} \quad 0 / 1.5 / 4]$ 60.0 CM: $1.79 \quad 52.7$ 135.0 CM: 1.7952 .6

$0>[2,65 / 6.61 \mathrm{C} 0 / 1,5 / 4]$ $60.0 \mathrm{cM}: \quad \begin{array}{rr}\mathrm{RHO} & \mathrm{PHI} \\ 1.86 & 48.7\end{array}$ 135.0 CM: $1.81 \quad 52.0$

$0>[2.65 / 6.61 \mathrm{C} \quad 0 / 1.5 / 4]$

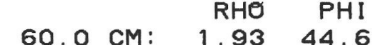
$135.0 \mathrm{CM}: \quad 2.00 \quad 39.9$ $0>[2.65 / 6.61 \mathrm{C} 0 / 1.5 / 4]$ $60.0 \mathrm{RHO}$ PHI $135.0 \mathrm{CM}: \quad 0.00 \quad 0.0$ 


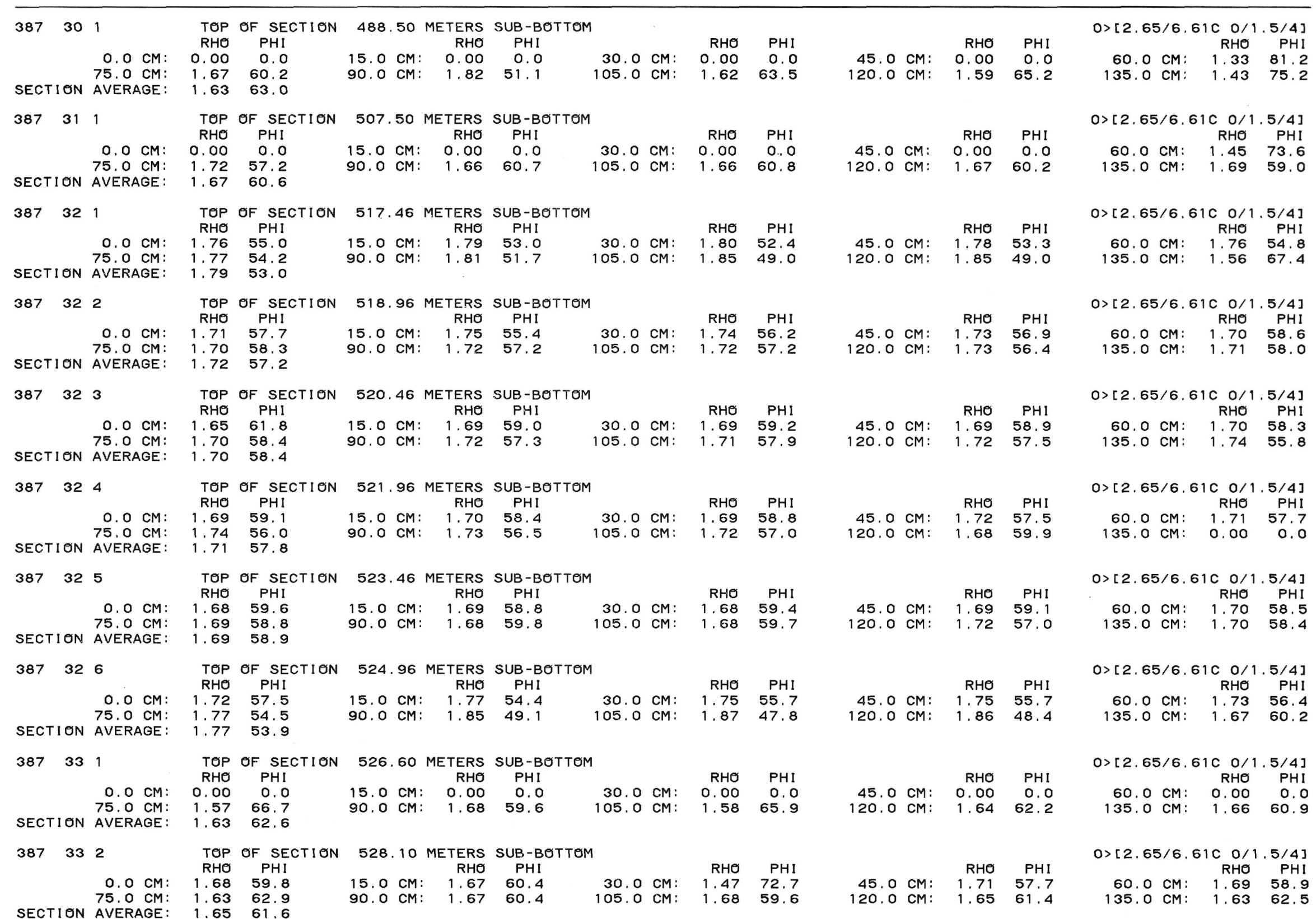


$38734 \quad 1$ TOP OF SECTION 536.20 METERS SUB-BOTTOM $0.0 \mathrm{cM}: \quad \mathrm{RHO}$ PHI $15.0 \mathrm{~cm}: \mathrm{RHO}^{\mathrm{PHI}} 30.0 \mathrm{~cm}$ RHO PHI

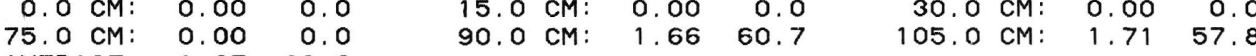
SECTION AVERAGE: 1.6760 .2

RHO PHI

70 METERS SUB-BOTTOM

$0.0 \mathrm{CM}: \quad 1.46 \quad 73.0$

75.O CM: $\quad 1.64 \quad 62.0$

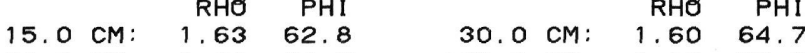
90.0 CM: $1.66 \quad 60.7 \quad 105.0 \mathrm{CM}: 1.8250 .8$

SECTION AVERAGE: 1.6263 .5

$387 \quad 35 \quad 2$

TOP OF SECTION $\mathbf{5 4 7 . 2 0}$ METERS SUB-BOTTOM

$0.0 \mathrm{cM}: \quad 1.6959 .2$

75.0 CM: 1.6561 .8

$\begin{array}{rrr}15.0 \mathrm{CM}: \quad 1.58 & \text { PHI } \\ 1.55 .9\end{array}$

SECTION AVERAGE: $1.68 \quad 59.5$ $\begin{array}{rrrrrr}90.0 \mathrm{CM}: & 1.71 & 57.8 & 105.0 \mathrm{cM}: & 1.74 & 56.3 \\ & & & & & \end{array}$

387353

TOP OF SECTION 548.70 METERS SUB-BOTTOM

15.0 $\mathrm{CM}:$ RHO PHI

$0.0 \mathrm{CM}: \quad 1.66 \quad 61.0$

15. O CM: $1.70 \quad 58.7$

30.0 CM: $\quad$ RHO PHI

75.0 CM: $1.71 \quad 57.7$

TOP OF SECTION 550.20 METERS SUB-BOTTOM

$387 \quad 35 \quad 4$

RHO PHI

$\begin{array}{rrr}0.0 \mathrm{CM}: & 1.66 & 60.9 \\ 75.0 \mathrm{CM}: & 1.70 & 58.3\end{array}$

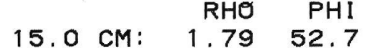

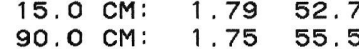

105.0 CM: 1.7257 .3

AVERAGE: $1.70 \quad 58.4$

$387 \quad 35 \quad 5$

TOP OF SECTION 551.70 METERS SUB-BOTTOM RHO PHI

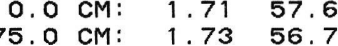

15.0 CM: $\quad 1.72 \quad 56.9$

90.0 CM: $1.71 \quad 57.6$

$\begin{array}{rrr}30.0 \mathrm{CM}: & \text { RHO } & \text { PHI } \\ 105.0 & 57.9\end{array}$

$\begin{array}{ll}.73 & 56.7 \\ .71 & 57.9\end{array}$

556. 80 METERS SUB-BOTTOM

TOP OF SECTIC RHO PHI

$387 \quad 36 \quad 2$

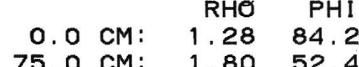

15.0 CM: $\quad$ RHO $\mathrm{PHI}$

$\begin{array}{rrr}30.0 \mathrm{CM}: & \mathrm{RHO} & \mathrm{PHI} \\ 1.47 & 72.9\end{array}$

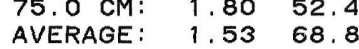

90.0 CM: 1.65 61.3

$105.0 \mathrm{CM}: \quad 1.62 \quad 63.2$

387363

TOP OF SECTION 558.30 METERS SUB-BOTTOM

RHO PHI

0.0 CM: 1.5866 .0

SECTION AVERAGE: $\quad 1.69 \quad 59.0$

$\begin{array}{rrrr}15.0 \mathrm{cM}: & \text { RHO } & \text { PHI } \\ 1.71 & 58.0\end{array}$

30.0 CM: $\begin{array}{rr}\mathrm{RHO} & \mathrm{PHI} \\ 1.60 & 64.5\end{array}$ 90.0 CM: $1.7456 .1 \quad 105.0 \mathrm{CM}: 1.75 \quad 55.4$

387364

TOP OF SECTION 559.80 METERS SUB-BOTTOM

RHO PHI

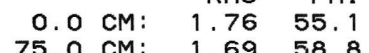

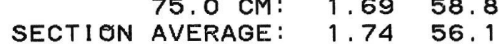

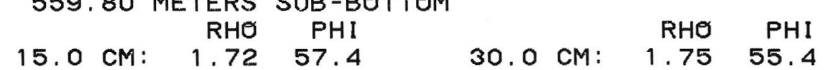

TOP OF SECTION 577.30 METERS SUB-BOTTOM

$387 \quad 37 \quad 3$

RHO PHI

$\begin{array}{rll}0.0 \mathrm{CM}: & 1.62 & 63.1 \\ 75.0 \mathrm{CM}: & 1.51 & 69.9\end{array}$

SECTION AVERAGE: 1.6760 .0 $\begin{array}{rrrrrr} & \text { RHO } & \text { PHI } & & \text { RHO } & \text { PHI } \\ 15.0 \mathrm{CM}: & 1.73 & 56.4 & 30.0 \mathrm{CM}: & 1.75 & 55.6 \\ 90.0 \mathrm{CM}: & 1.50 & 70.5 & 105.0 \mathrm{CM}: & 1.66 & 61.1\end{array}$ $\begin{array}{rrr}45.0 \mathrm{CM}: & 0.00 & \mathrm{PHI} \\ 120.0 \mathrm{CM}: & 1.66 & 61.2\end{array}$

45. $\mathrm{CM}: \quad \begin{array}{rr}\mathrm{RHO} & \mathrm{PHI} \\ 1.46 & 73.5\end{array}$

$120.0 \mathrm{CM}: \quad 1.70 \quad 58.3$

45. $\mathrm{CM}: \quad \begin{array}{r}\mathrm{RHO} \\ \mathrm{PHI}\end{array}$

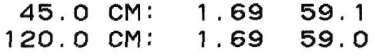

RHO $\mathrm{PHI}$

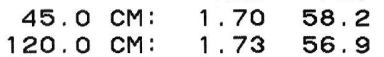

(1)

45. O CM: $\quad$\begin{tabular}{rrr} 
RHO & $\mathrm{PHI}$ \\
\hline & 61.5
\end{tabular}

$\begin{array}{rrr}\text { 45.0 CM: } & 1.65 & 61.5 \\ 120.0 \mathrm{CM}: & 1.76 & 55.0\end{array}$

45.0 CM: $\quad \begin{array}{rr}\text { RHO } & \text { PHI } \\ 120.73 & 56.5\end{array}$

$120.0 \mathrm{CM}: \quad 1.6760 .6$

45. O cM: $\begin{array}{rr}\text { RHO } & \text { PHI } \\ 1.50 & 70.9\end{array}$ $\begin{array}{rrr}\text { 120.0 CM: } & 1.56 & 67.4\end{array}$

45.0 CM: $\begin{array}{rr}\text { RHO } & \text { PHI } \\ 1.68 & 59.7\end{array}$

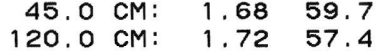

45.0 CM: $\begin{array}{rr}\text { RHO } & \text { PHI } \\ 1.73 & 56.7\end{array}$

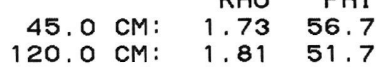

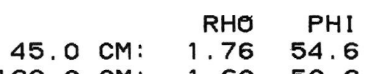

$0>[2.65 / 6.61 \mathrm{C} \quad 0 / 1.5 / 4]$ $60.0 \mathrm{cM}: \quad 0.00 \quad 0.0$ $135.0 \mathrm{CM}: 1.65 \quad 61.5$

0) $[2.65 / 6.61 \mathrm{C} \quad 0 / 1.5 / 4]$ $60.0 \mathrm{cM}: \quad \begin{array}{r}\mathrm{RHO} \\ 1.59\end{array} \quad \mathrm{PHI}$ $135.0 \mathrm{CM}: 1.5766 .5$

$0>[2.65 / 6.61 \mathrm{C} 0 / 1.5 / 4]$ $60.0 \mathrm{cM}: \quad \begin{array}{r}\text { RHO } \\ 1.70\end{array}$ $135.0 \mathrm{CM}: 1.7158 .0$

$0>[2.65 / 6.61 \mathrm{c} 0 / 1.5 / 4]$

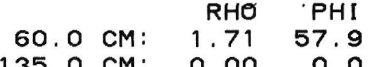

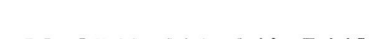

$0>[2.65 / 6.61 \mathrm{C} \quad 0 / 1.5 / 4]$

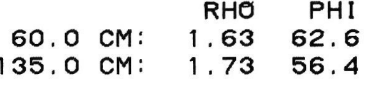

$0>[2.65 / 6.61 \mathrm{C} 0 / 1.5 / 4]$ $\begin{array}{rrr}60.0 \mathrm{CM}: & \text { RHO } & \text { PHI } \\ & 1.74 & 56.3\end{array}$ $0>[2.65 / 6.61 \mathrm{C} 0 / 1.5 / 4]$ $60.0 \mathrm{cM}: \quad \begin{array}{r}\mathrm{RHO} \\ \mathrm{PHI}\end{array}$ 135.0 CM: 1.6661 .2

$0>[2.65 / 6.61 \mathrm{C} \quad 0 / 1.5 / 4]$ $60.0 \mathrm{CM}: \quad \begin{array}{rr}\mathrm{RHO} & \mathrm{PHI} \\ 1.77 & 54.2\end{array}$ 135.0 CM: 1.7157 .8

$0>[2.65 / 6.61 \mathrm{C} 0 / 1.5 / 4]$ $60.0 \mathrm{~cm}: \quad \begin{array}{rr}\mathrm{RHO} & \mathrm{PHI}\end{array}$ $135.0 \mathrm{CM}: 1.7953 .2$

$0>[2.65 / 6.610 \quad 0 / 1.5 / 4]$ 
TABLE 1 - Continued

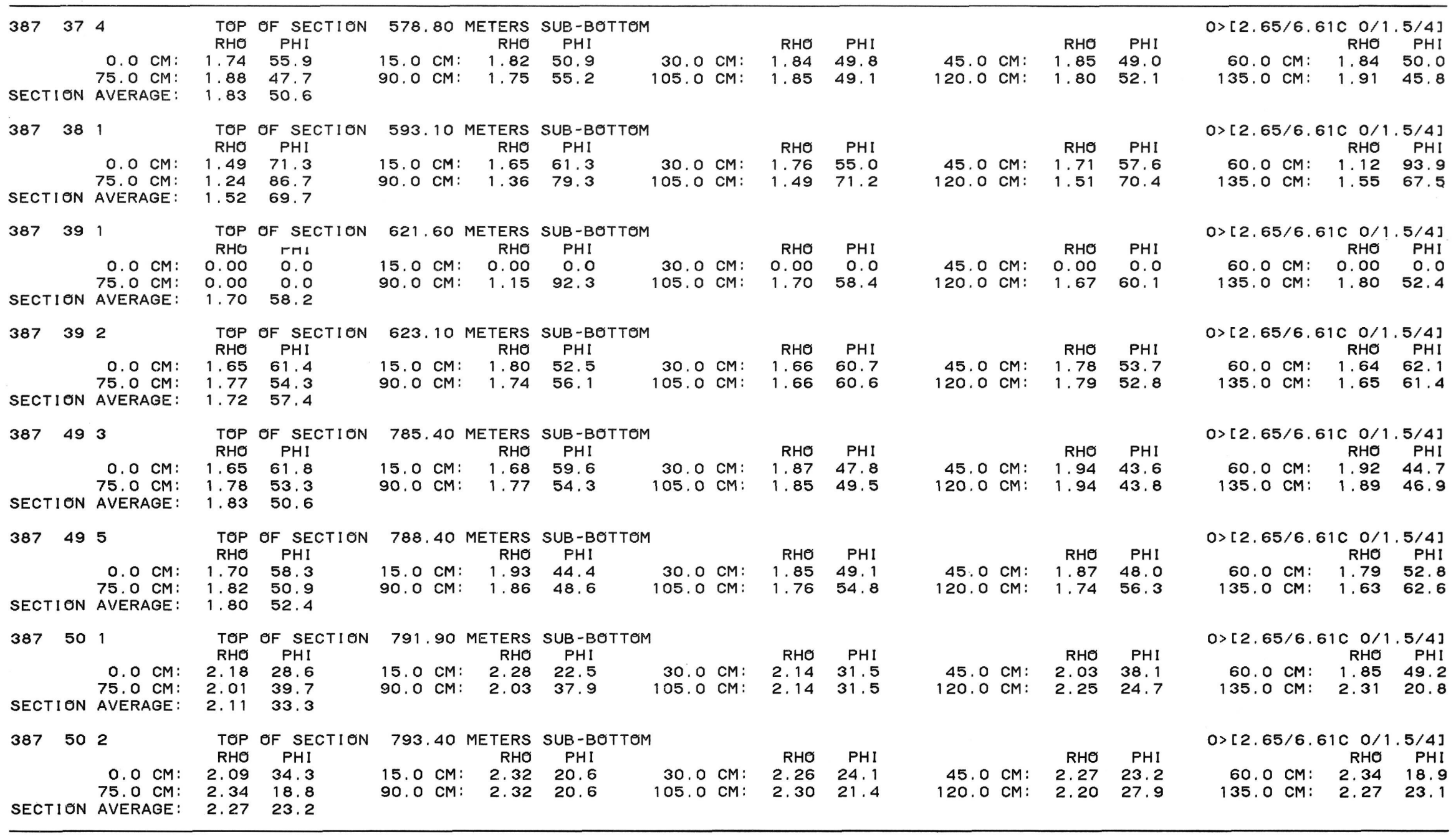

